\title{
Precision big bang nucleosynthesis with improved Helium-4 predictions
}

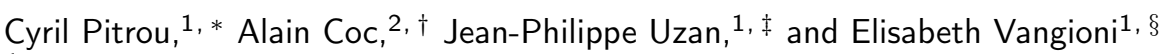 \\ ${ }^{1}$ Institut d'Astrophysique de Paris, CNRS UMR 7095, 98 bis Bd Arago, 75014 Paris, France \\ Sorbonne Université, Institut Lagrange de Paris, 98 bis Bd Arago, 75014 Paris, France \\ ${ }^{2}$ Centre de Sciences Nucléaires et de Sciences de la Matière (CSNSM), CNRS IN2P3, \\ Univ. Paris-Sud, Université Paris-Saclay, Bâtiment 104, F-91405 Orsay Campus France
}

(Dated: June 28, 2018)

Primordial nucleosynthesis is one of the three historical evidences for the big bang model, together with the expansion of the universe and the cosmic microwave background. There is a good global agreement between the computed primordial abundances of helium-4, deuterium, helium-3 and their values deduced from observations. Now that the number of neutrino families and the baryonic densities have been fixed by laboratory measurements or CMB observations, the model has no free parameter and its predictions are rigid. Since this is the earliest cosmic process for which we a priori know all the physics involved, departure from its predictions could provide hints or constraints on new physics or astrophysics in the early universe. Precision on primordial abundances deduced from observations has recently been drastically improved and reach the percent level for both deuterium and helium-4. Accordingly, the BBN predictions should reach the same level of precision. For most isotopes, the dominant sources of uncertainty come from those on the laboratory thermonuclear reactions. This article focuses on helium- 4 whose predicted primordial abundance depends essentially on weak interactions which control the neutron-proton ratio. The rates of the various weak interaction processes depend on the experimentally measured neutron lifetime, but also includes numerous corrections that we thoroughly investigate here. They are the radiative, zero-temperature, corrections, finite nucleon mass corrections, finite temperature radiative corrections, weak-magnetism, and QED plasma effects, which are for the first time all included and calculated in a self consistent way, allowing to take into account the correlations between them, and verifying that all satisfy detailed balance. Finally, we include the incomplete neutrino decoupling and claim to reach a $10^{-4}$ accuracy on the helium- 4 predicted mass fraction of $0.24709 \pm 0.00017$ (when including the uncertainty on the neutron lifetime). In addition, we provide a Mathematica primordial nucleosynthesis code that incorporates, not only these corrections but also a full network of reactions, using the best available thermonuclear reaction rates, allowing the predictions of primordial abundances of helium- 4 , deuterium, helium- 3 and lithium- 7 but also of heavier isotopes up to the $\mathrm{CNO}$ region.

\section{Contents}

\section{Introduction}

A. Observed abundances

B. Outlook on weak-rates corrections

C. Main eras of BBN

D. Resolution strategy and outline

II. Background thermodynamics

A. Thermodynamics in a FL spacetime

B. Plasma temperature

C. Baryon density

D. Cosmology and scale factor

E. QED corrections for the plasma thermodynamics

F. Incomplete neutrino decoupling

G. Effective description of neutrinos

III. Weak Interactions

A. General formulation

B. Infinite nucleon mass approximation

C. Calibration from free neutron decay rate

D. Neutron abundance and freeze-out

E. Radiative corrections at $T=0$

F. Finite temperature radiative corrections

*Electronic address: pitrou@iap.fr

${ }^{\dagger}$ Electronic address:

‡Electronic address: uzan@iap.fr

$\S$ Electronic address: vangioni@iap.fr
G. Finite nucleon mass corrections

H. Weak magnetism

I. Effect of incomplete neutrino decoupling

J. Total correction to the weak rates

IV. Nucleosynthesis

A. Thermonuclear reaction rates

B. General form

C. Nuclear network and reaction rates uncertainties

V. Numerical results

A. Overview of PRIMAT

B. Temperature of nucleosynthesis

C. Effect of corrections on abundances

D. Dependence on main parameters

E. Distribution of abundance predictions

F. Comparison with observations

VI. Cosmology with BBN

A. Cosmological perturbations

B. Measurement of baryon abundance from BBN

C. Neutrino chemical potential from BBN

D. Number of neutrinos

Conclusion

Acknowledgments

A. Thermodynamics

1. Thermodynamical quantities

2. Chemical potential of electrons

3. Nucleons at thermodynamical equilibrium 
4. Abundances at nuclear statistical equilibrium

B. Weak reactions rates

1. General expressions

2. Fokker-Planck expansion

3. Finite nucleon mass corrections

4. Weak-magnetism corrections

5. Mandelstam variables

6. Radiative corrections and Sirlin's universal function

7. Bremsstrahlung

8. Finite temperature radiative corrections

C. Nuclear reactions

1. Conventions for nuclear reaction rates

2. Baryonic density and nucleonic density

D. Numerical values

References

\section{INTRODUCTION}

Besides the universal spatial expansion and the cosmic microwave background (CMB) radiation, the third historical evidence for the hot big bang model comes from primordial, or big bang nucleosynthesis (BBN). During the first $\approx 20$ minutes of the Universe, when it was dense and hot enough for nuclear reactions to take place, BBN describes the production of the so called "light elements", ${ }^{4} \mathrm{He}, \mathrm{D},{ }^{3} \mathrm{He}$ and ${ }^{7} \mathrm{Li}$, together with only minute traces of heavier nuclei (see e.g. Coc and Vangioni (2017); Cyburt et al. (2016); Olive (2010); Patrignani and Particle Data Group (2016 and 2017 update); Steigman (2007) for recent reviews). The number of free parameters that entered in standard BBN has now been reduced to zero. Indeed, the number of light neutrino families is now known from the measurement of the $Z^{0}$ width by LEP experiments at CERN: $N_{\nu}=2.984 \pm 0.008$ (Patrignani and Particle Data Group, 2016 and 2017 update). The lifetime of the neutron entering in weak reaction rate calculations and many nuclear reaction rates have been measured in nuclear physics laboratories (Coc et al. 2015 , Cyburt et al., 2016; Descouvemont et al., 2004; Serpico et al. 2004). The last parameter to have been independently determined is the baryonic density of the Universe which is now deduced from the analysis of the anisotropies of the CMB radiation from the Planck satellite data (Ade et al. 2016). Hence, there is no more free parameter in standard BBN and the calculated primordial abundances are in principle only affected by the moderate uncertainties in nuclear cross sections. Keeping in mind that abundances span a range of nine orders of magnitude, the agreement between primordial abundances, either deduced from observations, or from primordial nucleosynthesis calculations, is an outstanding support to the hot big bang model (Cyburt et al., 2016 ). Hence, $\mathrm{BBN}$ is an invaluable tool for probing the physics of the early Universe, and it has been very efficient to constrain physics beyond the standard model. When we look back in time, it is the ultimate process for which we a priori know all the physics involved, given that it is very well tested in laboratories, so that departures from its predictions provide hints for new physics or astrophysics (Iocco et al., 2009; Mathews et al., 2017, Nakamura et al., 2017, Pospelov and Pradler, 2010).

Great progresses have been made in the precision of both observations and laboratory measurements. The precision on deuterium observations have now reached the percent level (Cooke et al., 2018), a precision hardly reached in nuclear physics measurements. The determination of the primordial abundance of ${ }^{4} \mathrm{He}$ has been reduced to less than $2 \%$ by the inclusion of an additional atomic infrared line (Aver et al. 2015). However, there is still a significant discrepancy on lithium, for which predictions are a factor of $\approx 3$ higher than observations. The previous paper Coc et al. (2015) investigated the uncertainties on $\mathrm{D} / \mathrm{H}$ predictions which directly reflects the experimental uncertainties on few reaction rates that have all been re-evaluated (Gómez Iñesta et al., 2017, Iliadis et al. 2016), leading to a small but significant decrease of the predicted deuterium abundance with reduced uncertainty. This review aims at reducing the uncertainties, this time on the ${ }^{4} \mathrm{He}$ abundance prediction which are dominated by theoretical uncertainties on the weak reactions that interconvert neutrons and protons.

To that goal, we implemented the BBN equations into a Mathematica code $^{1}$, in addition to the Fortran code, that has been used recently, e.g. in $\mathrm{Coc}$ et al. (2009); Coc et al. (2015); Coc and Vangioni (2010). This Fortran code originates from the model created at IAP by Elisabeth Vangioni (Vangioni-Flam et al. 2000) and further developed by Alain Coc (Coc et al., 2002). Hence, it was possible to cross-check the implementations and after tuning the parameters to reach similar precisions, verify that the results were virtually identical, and next to focus on nuclear reaction rate uncertainties. Leaving aside, the "lithium problem" that has not yet found a fully satisfactory solution (Fields, 2011), it has become essential to improve the precision on deuterium and ${ }^{4} \mathrm{He}$ primordial abundances ( ${ }^{3} \mathrm{He}$ is not pertinent here). As nuclear uncertainties affecting deuterium BBN have already been investigated recently (Coc et al., 2015, Gómez Iñesta et al., 2017; Iliadis et al. 2016), we will just summarize the situation. On the contrary the uncertainties impacting the ${ }^{4} \mathrm{He}$ abundance predictions are limited by the experimental value of the neutron lifetime (Patrignani and Particle Data Group, 2016 and 2017 update; Wietfeldt and Greene, 2011) but also by the numerous corrections that have to be introduced in the theoretical weak-interaction reaction rates. The main aim of this article is to calculate in details all these corrections. In our previous works, either only a fraction of them was taken into account (Coc et al., 2009, Coc and Vangioni, 2010 ) or were also supplemented by a final correction to

${ }^{1}$ PRIMAT : PRImordial MATter, freely available at http://www2. iap.fr/users/pitrou/primat.htm 
the ${ }^{4} \mathrm{He}$ abundance (Coc et al., 2014), based on other works (Dicus et al., 1982; Lopez and Turner, 1999).

With the now precise theoretical and observational determinations of both the deuterium and ${ }^{4} \mathrm{He}$ primordial abundances, it is now possible to better constrain the standard models of particle physics and cosmology and eventually provide hints of physics beyond the standard model.

We define $n_{i}$ as volume density of isotope $i$, and $n_{\mathrm{b}}$, the baryon or nucleon volume density. The (pseudo-)mass fraction of isotope $i$ is defined as

$$
X_{i} \equiv A_{i} \frac{n_{i}}{n_{\mathrm{b}}}
$$

where $A_{i}$ is the dimensionless mass integer number of nuclear physics, or baryon number of particle physics, (i.e. not the atomic mass). Baryon number conservation requires that $\sum_{i} A_{i} n_{i}=n_{\mathrm{b}}$ or $\sum_{i} X_{i}=1$. For ${ }^{4} \mathrm{He}$ it is customary to define

$$
Y_{\mathrm{P}} \equiv X^{4} \mathrm{He}
$$

and to introduce for convenience

$$
Y_{\mathrm{P}}^{(4)} \equiv 10^{4} Y_{\mathrm{P}}
$$

For other elements, it is customary to use the density ratio with ${ }^{1} \mathrm{H}$, that is $n_{i} / n^{1} \mathrm{H}$, abbreviated as $i / \mathrm{H}$.

\section{A. Observed abundances}

During the galactic evolution, massive stars are the main source of enrichment of the interstellar medium, when they explode as supernovae, out of which next generations of stars are born. In this process, they release matter, enriched in heavy elements that they have synthesized during the various phases of their evolution. Accordingly, the abundance of metals (elements heavier than helium) in star forming gas increases with time. The observed metallicity is therefore an indication of age: the older, the lower the metallicity. Hence, primordial abundances are extracted from observations of objects with low metallicity, but depending on their galactic chemical evolution, pristine abundances have to be derived from different classes of objects as detailed below.

\section{1. ${ }^{4}$ He observations}

After BBN, stars produce also ${ }^{4} \mathrm{He}$ and its primordial abundance can be measured thanks to observations in HiI (ionized hydrogen) regions inside compact blue galaxies. It is thought that galaxies are formed by the merging of such dwarf galaxies, in a hierarchical structure formation paradigm, hence these are considered to be more primitive. First, for each HiI region, the ${ }^{4} \mathrm{He}$ abundance has to be determined within a model aiming at reproducing the observed ${ }^{4} \mathrm{He}$ emission lines, that also depends on parameters like e.g. the electron density. Then, to account for stellar production, the ${ }^{4} \mathrm{He}$ deduced abundances should be extrapolated to zero metallicity. Recently, the observation (Izotov et al. 2014 ) of an additional atomic infrared line $(\lambda 10830)$ in 45 low-metallicity HII regions have allowed to better constrain the thermodynamic conditions that prevail in the emission regions. After selecting 28 object, Izotov et al. (2014) obtained $Y_{\mathrm{P}}=0.2551 \pm 0.0022$ for the ${ }^{4} \mathrm{He}$ mass fraction. However, Aver et al. (2015) (see also Cyburt et al. (2016)), starting from the same observational data made a stricter selection, based, in particular on goodness of fit for the emission model of each object. For the selected 16 objects, the uncertainties were so reduced that a slope in the $Y_{p}$ versus metallicity data could be considered. After extrapolation to zero metallicity, Aver et al. (2015) obtained,

$$
Y_{\mathrm{P}}=0.2449 \pm 0.0040,
$$

that we use here.

\section{Deuterium observations}

Deuterium is a very fragile isotope. It can only be destroyed after BBN thanks to stellar evolution. The deuterium abundance closest to primordial abundance is determined from the observation of a few cosmological clouds (absorbers) at high redshift on the line of sight of distant quasars (emitters). Figure 1 shows the observed $\mathrm{D} / \mathrm{H}$ values as a function of the redshift of the absorber. Until recently, the distribution of $\mathrm{D} / \mathrm{H}$ observations showed a significant scatter (see e.g. Pettini and Cooke (2012)). It allowed Olive et al. (2012) to adopt a weighted mean of $\mathrm{D} / \mathrm{H}=(3.02 \pm 0.23) \times 10^{-5}$ without excluding an upper limit of $\mathrm{D} / \mathrm{H}=4 \times 10^{-5}$. This is not possible anymore thanks to the recent new observations or reanalyses of existing data (Balashev et al., 2016 , Cooke et al., 2014, 2016, 2018; Riemer-Sørensen et al., 2017) that now display a plateau as a function of redshift (and metallicity) with a very small scatter (Fig. 1). Moreover, in Dvorkin et al. (2016), the comparison with the available measurements is consistent with the cosmic merger tree model of structure formation. It is shown that at redshift higher than 2 (see their Fig. 2), the dispersion in the cosmic deuterium abundance is very tiny leading to think that at these redshifts the observation of the D abundance is probably primordial. Hence, we adopt the new recommended value provided by Cooke et al. (2018):

$$
\mathrm{D} / \mathrm{H}=(2.527 \pm 0.030) \times 10^{-5},
$$

lower and with smaller uncertainties than in previous determinations $^{2}$. If such a precision of $\sim 1 \%$ in observations

\footnotetext{
2 A recent reanalysis and new $\mathrm{D} / \mathrm{H}$ observations towards the Q1009+2956 quasar (Zavarygin et al. 2017. 2018) provided a
} 
is confirmed, great care should be paid to nuclear cross sections affecting deuterium nucleosynthesis.

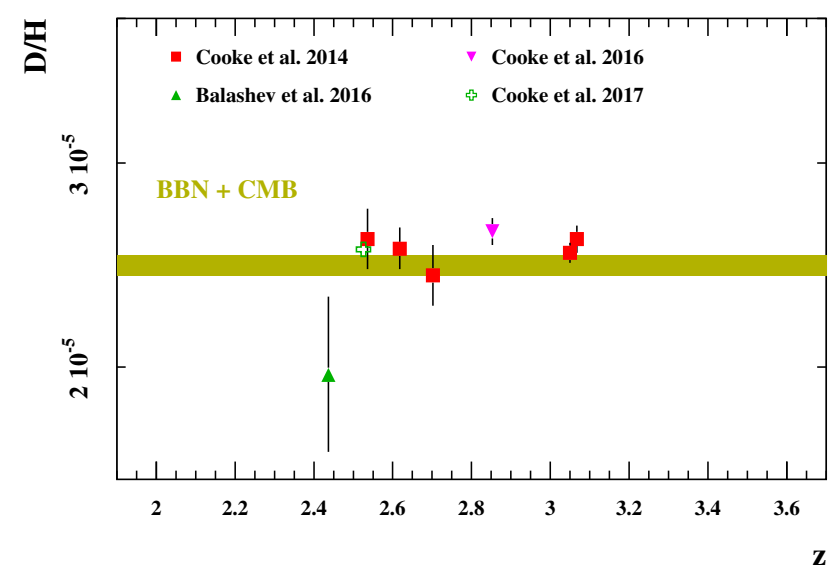

FIG. $1 \mathrm{D} / \mathrm{H}$ observations, as a function of the redshift of the absorber. These most recent observations (Cooke et al. 2014. 2016, 2018) have very small error bars and show very few dispersion compared to previous determinations (except Balashev et al. (2016)), and are in fair agreement with our BBN calculations when using Planck (Ade et al. 2016) baryonic density.

\section{3. ${ }^{3} \mathrm{He}$ observations}

Contrary to the case of ${ }^{4} \mathrm{He}$, stars can both produce and destroy ${ }^{3} \mathrm{He}$, so that the evolution of its abundance in time is not known precisely (Vangioni-Flam et al., 2003 ). Since observing helium is difficult, and given the small ${ }^{3} \mathrm{He} /{ }^{4} \mathrm{He}$ ratio, ${ }^{3} \mathrm{He}$ was only observed in our Galaxy and the bounds obtained are ${ }^{3} \mathrm{He} / \mathrm{H}=(0.9-1.3) \times 10^{-5}$, keeping in mind that this is an upper limit extracted from a single object (Bania et al. 2002). However, the next generation of $30+\mathrm{m}$ telescope facilities may allow to extract the ${ }^{3} \mathrm{He} /{ }^{4} \mathrm{He}$ ratio from observations of extragalactic metal poor HII regions (Cooke, 2015).

\section{4. ${ }^{7} \mathrm{Li}$ observations}

${ }^{7} \mathrm{Li}$ is peculiar because it has three distinct sources: BBN but also spallative nuclear reactions between galactic cosmic rays and the interstellar medium, and a stellar source (Asymptotic giant branch stars and novae) (Fu et al. 2018). For instance, recent observations (Izzo

new value of $\mathrm{D} / \mathrm{H}=\left(2.48_{-0.35}^{+0.41}\right) \times 10^{-5}$ with a limited precision (17\%). If included, it shifts the abundance downward to $\mathrm{D} / \mathrm{H}=$ $(2.545 \pm 0.025) \times 10^{-5}$ et al., 2015, Tajitsu et al., 2016) have confirmed Li production by novae, at a level even higher than model predictions (Hernanz et al. 1996). Hence, after BBN, ${ }^{7} \mathrm{Li}$ can be produced but can also easily be destroyed in the interior of stars by proton capture at temperatures as low as $2.5 \mathrm{MK}$.
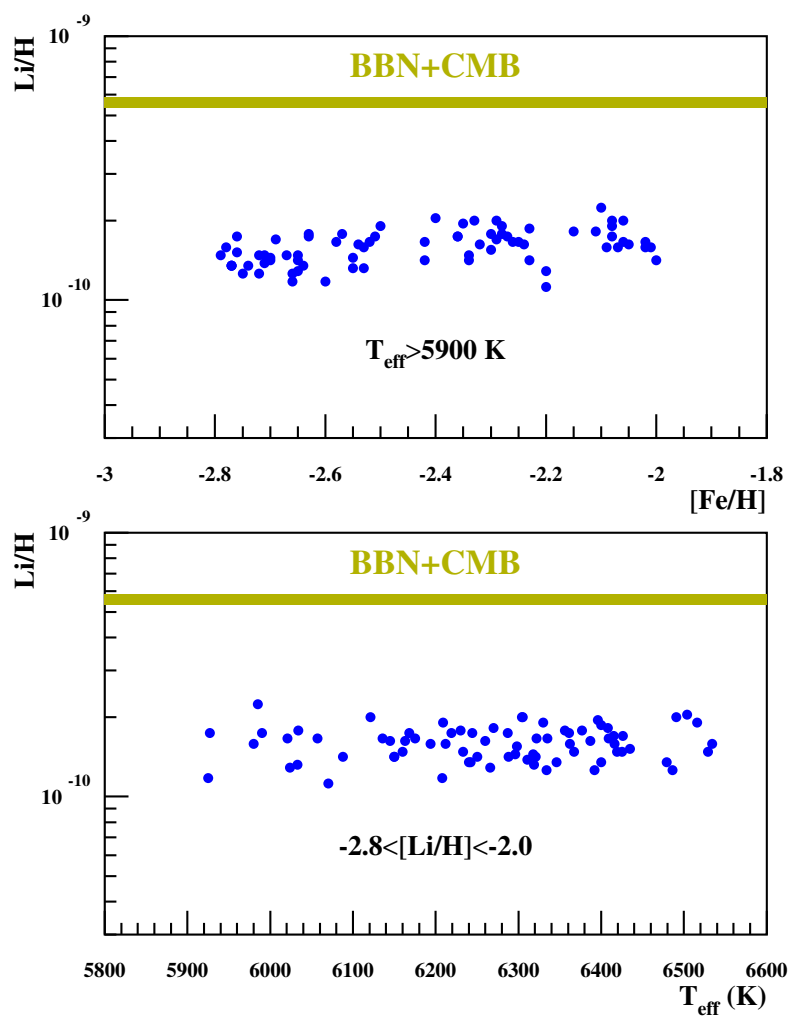

FIG. $2 \mathrm{Li} / \mathrm{H}$ observations, as a function of metallicity ${ }^{3}$ and effective temperature. Data (Aoki et al., 2009, Asplund et al. 2006. Bonifacio et al. 2007, Charbonnel and Primas, 2005. Hosford et al., 2009; Meléndez et al., 2010, Sbordone et al. 2010| Schaeuble and King | 2012 |Scholz et al., 2015) come from a compilation by Spite et al. $(2012,2015)$.

The life expectancy of stars with masses lower than our Sun is larger than the age of the Universe so that very old such stars can still be observed in the halo of our Galaxy. In this context, lithium can be observed at the surface of these stars and its abundance was found to be independent of metallicity and effective temperature in the ranges $-2.4 \leq[\mathrm{Fe} / \mathrm{H}] \leq-1.4^{3}$ (i.e. between $\approx 4 \times 10^{-3}$ and $4 \times 10^{-1}$ of the solar metallicity) and $5700 \leq T_{\text {eff }} \leq 6800 \mathrm{~K}$ (Fu et al. 2018). This plateau was discovered by François

\footnotetext{
${ }^{3}$ Logarithm of the ratio, relative to its solar value: $[X / Y] \equiv$ $\log \left((X / Y) /\left(X_{\odot} / Y_{\odot}\right)\right)$.
} 
and Monique Spite (Spite and Spite, 1982) and this constant $\mathrm{Li}$ abundance is interpreted as corresponding to the BBN ${ }^{7} \mathrm{Li}$ production. The thinness of the "Spite plateau" has generally been considered as an indication that surface $\mathrm{Li}$ depletion may not have been very effective so that it should reflect the primordial value. However, as shown in Fig. 2, there is a discrepancy of a factor $\approx 3$ between the BBN predicted value and the lithium abundance derived in metal-poor main-sequence (MS) stars. This discrepancy could be alleviated if the stars observed today had undergone photospheric depletion of lithium. Different observations in globular clusters (see Gruyters et al. (2016)) and stellar scenarios concerning the formation of the early metal poor stars have been developed, $(\mathrm{Fu}$ et al. 2015) trying to follow lithium evolution in metalpoor stars, from pre-main sequence to the Spite plateau. $\mathrm{Li}$ evolution could affect its abundance by introducing the effects of convective overshooting, atomic diffusion and mixing (Richard et al., 2005 ). In this context, ${ }^{7} \mathrm{Li}$ could be depleted and it could be possible to move closer to the observed Li Spite plateau. Indeed, several globular clusters (NGC 6397, 6752, M30) show that the discrepancy could be reduced by atomic diffusion leading to a factor of 1.6 instead 3 (Michaud et al., 1984). Finally the pre-MS depletion can be efficient and this is presently an open question. Note that recent lithium observations (Howk et al. 2012 ) have been done in the Small Magellanic Cloud which has a quarter of the sun's metallicity and a $\mathrm{Li}$ abundance nearly equal to the BBN predictions.

Recently, Reggiani et al. (2017) have constrained cosmic scatter in the galactic halo using a differential analysis of metal poor for several elements. Regarding lithium, they find a very low scatter $(0.04 \mathrm{dex})$ and a mean value $1.86 \times 10^{-10}$ which is compatible with other studies. We adopt here the analysis of Sbordone et al. (2010), namely ${ }^{4}$

$$
{ }^{7} \mathrm{Li} / \mathrm{H}=(1.58 \pm 0.3) \times 10^{-10} .
$$

\section{B. Outlook on weak-rates corrections}

Given the observational precision on ${ }^{4} \mathrm{He}$, we aim at predictions with a precision better than $0.1 \%$. This amounts to considering all effects which affect $Y_{\mathrm{P}}^{(4)}$ by units, and to treat carefully all effects which modify the abundance by an order $10^{-4}$. $Y_{\mathrm{P}}$ is almost exclusively controlled by weak reactions, because nearly all neutrons end up in ${ }^{4} \mathrm{He}$. By varying artificially the weak rates $(\Gamma)$ we find the relation ${ }^{5}$

$$
\frac{\delta Y_{\mathrm{P}}}{Y_{\mathrm{P}}} \simeq-0.73 \frac{\delta \Gamma}{\Gamma}
$$

\footnotetext{
4 All uncertainties are given with one standard deviation.

${ }^{5}$ Using Eq. 146a and $\Gamma \propto \tau_{\mathrm{n}}^{-1}$ from Eq. 91.
}

Hence we need to focus on all corrections which affect the weak rates by $10^{-4}$ or more, on top of the experimental uncertainty on the neutron lifetime.

As for ${ }^{2} \mathrm{H}$ and ${ }^{3} \mathrm{He}$, we aim at predictions of order $10^{-3}$ if we leave aside the uncertainty in nuclear rates, and of order of a few $10^{-2}$ when including uncertainties in nuclear rates. In all our computations, we must also make sure to maintain numerical errors much below $10^{-3}$.

There are many effects to take into account for the weak rates and we must also consider their possible couplings since they cannot always be summed linearly. These effects have several origins, namely

1. radiative corrections,

2. finite nucleon mass corrections,

3. finite temperature radiative corrections,

4. weak-magnetism,

5. QED plasma effects,

6. incomplete neutrino decoupling.

Radiative corrections correspond to the contribution of virtual photons in weak reactions, together with the emission of photons in the final state (bremsstrahlung) from the electron line. They are typically order $10^{-2}$ effects (see $\S$ V.C) because of the value of the fine-structure constant $\alpha_{\mathrm{FS}} \simeq 1 / 137$. They are well established in the context of neutron beta decay (Czarnecki et al. 2004), and even with some resummed effects which are higher orders in $\alpha_{\mathrm{FS}}$ for increased precision (Ivanov et al. 2013). In the context of BBN, these effects were originally estimated by Dicus et al. (1982).

Finite nucleon mass correction correspond to the effect of nucleon recoil and nucleon thermal distribution of velocity in the theoretical computation of the weak rates. These corrections are also of order $10^{-2}$ (see $\S$ V.C) even if smaller than radiative corrections. Wilkinson (1982) provided a comprehensive list of these effects in the context of neutron beta decay, and Seckel (1993) reviewed these corrections in the context of BBN. These were later estimated numerically by Lopez et al. (1997) using a Monte-Carlo estimation of multidimensional integrals. We introduce a new efficient method which relies on a Fokker-Planck expansion in the energy transfer, and which relies only on one-dimensional integrals.

Finite temperature radiative corrections correspond to the interactions with the bath of electrons and positrons during weak reactions. They lead to a long subject of controversy in the literature. They were initially computed in Dicus et al. (1982) and Cambier et al. (1982). Discrepancies between these approaches were analyzed by Kernan (1993) and a numerical estimation was provided by Lopez and Turner (1999). However, Brown and Sawyer (2001) pointed incoherences and the lack of detailed balance, and they proposed to set the 
finite temperature radiative correction on firm ground by providing a comprehensive theoretical computation from finite-temperature quantum field theory. We find, and this is new, that to be complete and satisfy detailed balance, one must also consider corrections to the bremsstrahlung effects and add them to finite temperature corrections. Overall, when finite-temperature corrections and bremsstrahlung corrections are added, they almost perfectly cancel, leaving $Y_{\mathrm{P}}^{(4)}$ nearly unchanged (see $\S \mathrm{V} . \mathrm{C}$ ).

Weak-magnetism which arises from the internal structure of nucleons is part of finite mass corrections and is order $10^{-3}$ (see $\S$ V.C).

Quantum Electrodynamics (QED) is responsible for two effects. First the interaction with the plasma modifies the electron mass. However this effect is strictly speaking part of finite temperature radiative corrections in our computations. Second, QED effects modify the thermodynamics of the plasma, that is it modifies the pressure and energy density. We find that this effect, taken alone, results in a negligible modification of $Y_{\mathrm{P}}^{(4)}$ which is of order $10^{-5}$ (see $\S$ V.C). However it affects the number of effective neutrinos for subsequent cosmology such as the physics of cosmic microwave background (CMB).

Incomplete neutrino decoupling corrections occur when electrons and positrons annihilate, because neutrinos are not fully decoupled from the plasma and some annihilations end up heating the neutrino bath. This effect increases the neutrino temperature and produces distortions in their spectrum. In order to track the neutrinos spectral distortions, it is necessary to use the full machinery of coupled Boltzmann equations, especially when considering neutrino oscillations. Since this effect also modifies the effective number of neutrinos for the subsequent cosmology, several authors among which Dolgov et al. (1997); Grohs et al. (2016); Mangano et al. (2005); de Salas and Pastor (2016) focused on it. We shall use a fit taken from Pisanti et al. (2008) for the heating rate of neutrinos. Eventually we find that this correction is of order $10^{-3}$ (see $\S$ V.C.).

All these effects are detailed in $\S$ III.

\section{Main eras of BBN}

During BBN, one can distinguish different eras depending on the dominant physical processes.

1. For plasma temperatures $T$ in the range $2 \mathrm{MeV} \lesssim$ $T \lesssim \mathrm{GeV}$, nucleons are formed and their density is only affected by expansion. Neutrinos are still in thermal equilibrium with the plasma (electronpositrons and protons), that is $T_{\gamma}=T_{\nu}$ and the neutron to proton ratio $X_{n} / X_{p}$ is enforced to be the thermodynamical equilibrium value since weak interactions interconvert efficiently neutrons in protons and vice-versa, through the reactions (68).

2. In the range, $0.8 \mathrm{MeV} \lesssim T \lesssim 2 \mathrm{MeV}$ neutrinos have essentially decoupled but weak interactions maintain neutrons and proton in thermodynamical equilibrium. However at weak-interactions freeze-out temperature $T_{F} \simeq 0.8 \mathrm{MeV}$, defined by the equality between weak interaction rates and the cosmological expansion rate, the abundance of neutrons are mostly affected by neutron beta decay. In practice, freeze-out is not instantaneous and the neutron abundance is subject only to neutron beta decay around $T_{F} \simeq 0.28 \mathrm{MeV}$. The abundance of neutrons is then about $X_{n} \simeq 0.17$ and it is eventually reduced to $X_{n}^{\text {Nuc }} \simeq 0.125$ by beta decay when nucleosynthesis starts (see Fig. 3).

3. Around $0.5 \mathrm{MeV}$, electrons and positrons annihilate and heat up the photon bath, resulting in a different temperature between photons and neutrinos $\left(T_{\gamma}>T_{\nu}\right)$. This affects directly the expansion history of the Universe since the energy content of massive particles (electrons and positrons) is replaced by massless particles (photons), but it also affects weak-interaction rates since this is concomitant with the freeze-out period.

4. As long as $T \gtrsim 0.078 \mathrm{MeV}$, deuterium dissociation is too efficient to allow for deuterium synthesis. Even though the binding energy of deuterium is about $2.2 \mathrm{MeV}$, deuterium is efficiently destroyed by the high-energy tail of the Bose-Einstein distribution of photons, because the ratio between baryon number density and photon number density $\eta$ is smaller than $10^{-9}$.

5. Below $T \lesssim T_{\text {Nuc }}=0.078 \mathrm{MeV}$, deuterium can be formed. Then since the binding energy per nucleon of ${ }^{4} \mathrm{He}$ is much larger than for deuterium, a network of reactions ends up in producing nearly only ${ }^{4} \mathrm{He}$ and very tiny amounts of other light elements. Since ${ }^{4} \mathrm{He}$ is made of two neutrons and two protons, the (pseudo-)mass abundance satisfies $Y_{\mathrm{P}} \simeq 2 X_{n}^{\text {Nuc }}$, hence leading to a final value $Y_{\mathrm{P}} \simeq 0.25$. Nucleosynthesis is completely over for all elements when $T \lesssim 0.01 \mathrm{MeV}$ or $T \lesssim 10^{8} \mathrm{~K}$ but for prediction with $10^{-3}$ precision on deuterium, we found that we must wait until $T \lesssim 6 \times 10^{7} \mathrm{~K}$.

\section{Resolution strategy and outline}

This outline of nucleosynthesis implies that we do not need to solve jointly the abundance of all species at all times. Indeed, given that the matter-radiation equivalence occurs around $T \simeq 3000 \mathrm{~K}$, this means that baryonic matter accounts for less than $10^{-4}$ of the total energy 


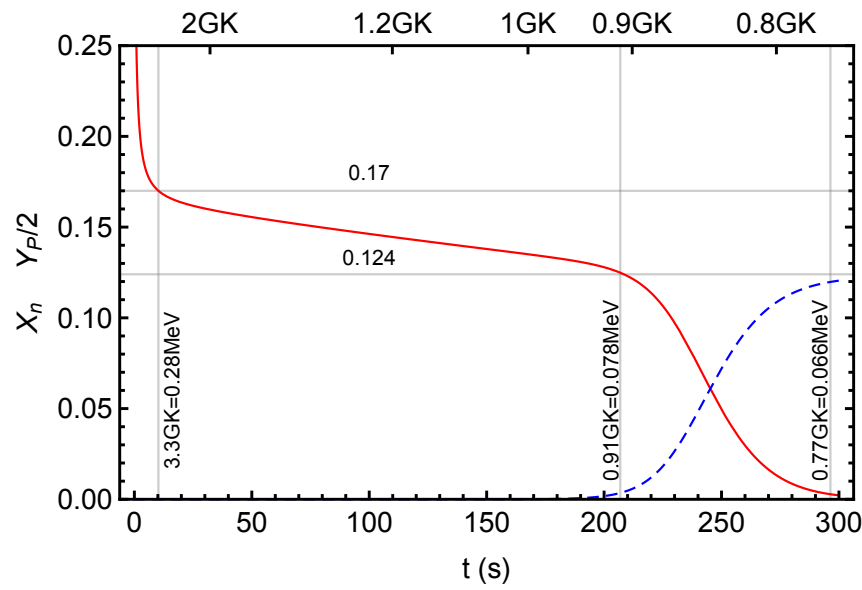

FIG. 3 Evolution of $X_{n}$ (red continuous line with nuclear reactions and red dotted line without nuclear reactions) and $Y_{\mathrm{P}} / 2$ (blue dashed line).

density at the end of BBN and even less during freezeout. In principle, one should account for the fact that neutron abundance evolves even though the nucleonic density (protons and baryons) is only affected by cosmic expansion. Since the mass difference between neutrons and protons is of order $10^{-3}$ of their rest mass energy, we can ignore this effect when evaluating the total energy density of the Universe. Similarly, given that the binding energy in nuclei is around or less than $10^{-2}$ of the rest mass energy, we can also consider to a very good approximation that nuclear reactions do not affect the baryon energy density. Hence it is possible to compute the evolution of the cosmological background, without having to compute in details the abundance of neutrons nor the details of nuclear reactions. In $\S \mathrm{II}$ we study the thermodynamics of all species and the dynamics of the cosmic expansion. Then in $\S$ III we focus on weak interactions and detail all corrections. Since the weak interaction rates depend on the plasma of electrons, positrons and neutrinos only, they can be studied ignoring nuclear reactions. Finally in $\S$ IV we focus on nuclear reactions and we present the results obtained when coupling the background dynamics, the weak interactions and the nuclear reactions. Most technical details can be found in the appendices for ease of reading. We use natural units in all expressions, that is we work in units in which

$$
k_{B}=\hbar=c=1,
$$

except when we judge instructive to write them explicitly.

\section{BACKGROUND THERMODYNAMICS}

We consider a homogeneous and isotropic cosmology, more precisely a flat Friedmann-Lemaître (FL) spacetime characterized by the scale factor $a(t)$ where $t$ is the cosmic time. The Hubble expansion rate is $H=\dot{a} / a$, where a dot indicates a derivative with respect to $t$. Since cos- mological perturbations are of order $10^{-5}$, it is fully justified to ignore their effect and consider an homogeneous cosmology given our precision goal.

\section{A. Thermodynamics in a FL spacetime}

\section{Distribution function and Boltzmann equation}

Relativistic species, which encompass neutrinos, photons and electrons with positrons during the relevant $\mathrm{BBN}$ era, are best described with a distribution function $f(t, p)$ for each species, and where the dependence is only on the magnitude of momenta $p=\sqrt{E^{2}-m^{2}}$ given the symmetries of the FL spacetime. This description is valid even out of thermodynamical equilibrium. The distribution function satisfies the general Boltzmann equation in a FL spacetime

$$
L[f] \equiv \frac{\partial f}{\partial t}+\dot{p} \frac{\partial f}{\partial p}=C[f], \quad \dot{p}=-H p .
$$

We have used that $p \propto 1 / a$ because of cosmic expansion and $C[f]$ is the collision term of the species under scrutiny. In order to relate this description to the fluid description, thermodynamical quantities such as number density $n$, energy density $\rho$ and pressure $P$, can be formed from the distribution function as summarized in App. A.

\section{Number density evolution}

From the definition of thermodynamic quantities (Eqs. A1), considering $\int L[f] 4 \pi p^{2} \mathrm{~d} p /(2 \pi)^{3}$ with the Boltzmann equation (9) leads after integration by parts to the number conservation equation

$$
\dot{n}+3 H n=\mathcal{J}, \quad \mathcal{J} \equiv \int C[f] \frac{4 \pi p^{2} \mathrm{~d} p}{(2 \pi)^{3}} .
$$

$\mathcal{J}$ is the net creation rate of particles per unit of physical volume. When the collision term vanishes or when it conserves the number of particles because it describes elastic scattering,

$$
\dot{n}+3 H n=0 \quad \Rightarrow \quad \frac{\mathrm{d}\left(n a^{3}\right)}{\mathrm{d} t}=0 .
$$

In that case, for a comoving volume $a^{3}$, the total number of particles $N \equiv n a^{3}$ remains constant.

\section{Energy density evolution}

Similarly, starting from the definition of thermodynamic quantities (Eqs. A1), and considering $\int L[f] E 4 \pi p^{2} \mathrm{~d} p /(2 \pi)^{3}$ with the Boltzmann equation (9), leads after integration by parts (and using $E \mathrm{~d} E=p \mathrm{~d} p$ ) to the energy conservation equation

$$
\dot{\rho}+3 H(\rho+P)=\dot{q}, \quad \dot{q} \equiv \int C[f] \frac{4 \pi E p^{2} \mathrm{~d} p}{(2 \pi)^{3}} .
$$


$\dot{q}$ is the volume heating rate. For massless particles, such as photons or neutrinos (which can be considered massless during the $\mathrm{BBN}$ ), $E=p$ and $P=\rho / 3$, implying that when the collision term is vanishing

$$
\dot{\rho}+4 H \rho=0 \Rightarrow \frac{\mathrm{d}\left(\rho a^{4}\right)}{\mathrm{d} t}=0 .
$$

Eqs. (12) and (13) are general and do not rely on a specific distribution function.

\section{Entropy evolution}

Volume entropy is defined (see e.g. Grohs et al. (2016)) from the distribution function by

$$
s=-\int S_{\mathrm{B}}(f) \frac{4 \pi p^{2} \mathrm{~d} p}{(2 \pi)^{3}},
$$

where the Boltzmann entropy is defined as

$$
S_{\mathrm{B}}(f) \equiv[f \ln f \pm(1 \mp f) \ln (1 \mp f)]
$$

with upper (lower) sign for fermions (bosons). Using the identity

$$
\partial_{p}\left[S_{\mathrm{B}}(f)\right]=\ln \left(\frac{f}{1 \mp f}\right) \partial_{p} f
$$

and multiplying Eq. (9) by $S_{\mathrm{B}}(f)$ we get after integration by parts

$$
\dot{s}+3 H s=-\int C[f] \ln \left(\frac{f}{1 \mp f}\right) \frac{4 \pi p^{2} \mathrm{~d} p}{(2 \pi)^{3}},
$$

which dictates the evolution of volume entropy. If there are no collisions, it is only affected by dilution as $s \propto 1 / a^{3}$ such that the total entropy in a given comoving volume, $S \equiv s a^{3}$, is conserved $(\dot{S}=0)$.

\section{Local thermodynamical equilibrium}

In case of local thermodynamical equilibrium (LTE), fermions (bosons) follow a Fermi-Dirac (Bose-Einstein) distribution A3 and

$$
\ln \left(\frac{f}{1 \mp f}\right)=\frac{\mu-E}{T},
$$

where $\mu$ is the chemical potential. Hence the evolution of volume entropy for a given species satisfies, using Eqs. (10) and 17),

$$
\dot{s}+3 H s=\frac{\dot{q}}{T}-\frac{\mu}{T}(\dot{n}+3 H n),
$$

with $\dot{q}$ defined in Eq. (12). From Eq. 19 multiplied by $a^{3}$, we recover the usual thermodynamical identity

$$
T \dot{S}=\dot{Q}-\mu \dot{N}
$$

with $\dot{Q} \equiv \dot{q} a^{3}$. Hence, the entropy for a given species is conserved if there is no heat exchange, that is no interactions, and either a vanishing chemical potential (in practice $\mu \ll T)$ or conservation of the number of particles (i.e. $\dot{N}=0$ ). From the conservation of the total stress-energy tensor, we must have $\sum_{i} \dot{q}_{i}=0$, that is there can be no global production of heat. Hence, in case of local thermodynamical equilibrium, the total entropy is conserved whenever for all species either the chemical potential is negligible $\mu_{i} \ll T$ or the number of particles is conserved.

If there is LTE, entropy for a given species $i$ can then be linked to other thermodynamical quantities thanks to

$$
s_{i}=\frac{P_{i}+\rho_{i}-\mu_{i} n_{i}}{T_{i}} .
$$

The total entropy is obtained from the extensivity of $\rho$ and $n$, given that pressure and temperature of all species are equal at equilibrium

$$
s=\sum_{i} \frac{P_{i}+\rho_{i}}{T}+\sum_{i} \frac{\mu_{i} n_{i}}{T}=\frac{\rho+P}{T}+\sum_{i} \frac{\mu_{i} n_{i}}{T} .
$$

During nucleosynthesis, given the very low value of the baryon-to-photon ratio $\eta$ (see $\S$ II.C below), we can totally ignore the entropy of protons and neutrons. This amounts to neglecting the electrons, positrons and neutrinos created and destroyed in weak interactions, and any energy exchange with these particles and the photons. Hence we can focus only on the thermodynamics of electrons, positrons, photons and neutrinos. During nucleosynthesis, neutrinos are nearly fully decoupled from other species and their entropy is separately conserved if we can assume that they are totally decoupled. See \$II.F for the small modifications induced by incomplete decoupling (ID).

Conversely, electrons, positrons and photons are tightly coupled by electromagnetic interactions and they behave collectively as a plasma whose common temperature is defined as $T$. Photons decouple from electrons only around recombination much later when the CMB is formed and $T \lesssim \mathrm{eV}$. Due to electron-positron annihilations, the number of particles in the plasma is not conserved, and it is crucial that we can neglect the chemical potential of plasma particles to claim that the plasma entropy is conserved. The chemical potential of photons is always vanishing due to processes which do not conserve the number of photons so we need only to make sure that the chemical potential of electrons and positrons can be neglected. In App. A.2, we evaluate these chemical potentials and show they are completely negligible, implying that we can use entropy conservation.

For convenience we define dimensionless reduced energy density, pressure, number density and entropy as

$$
\bar{n}_{i} \equiv \frac{n_{i}}{T_{i}^{3}} \quad \bar{P}_{i} \equiv \frac{P_{i}}{T_{i}^{4}} \quad \bar{\rho}_{i} \equiv \frac{\rho_{i}}{T_{i}^{4}} \quad \bar{s}_{i} \equiv \frac{s_{i}}{T_{i}^{3}}
$$


such that the relation (21) reads simply in case of vanishing chemical potential

$$
\bar{s}_{i}=\bar{\rho}_{i}+\bar{P}_{i} .
$$

For photons the reduced thermodynamic variables do not depend on temperature and are constants when there is LTE. We find

$$
\begin{aligned}
& \bar{n}_{\gamma}=\frac{2}{2 \pi^{2}} I_{-}^{(1,1)}=\frac{2 \zeta(3)}{\pi^{2}} \\
& \bar{\rho}_{\gamma}=\frac{2}{2 \pi^{2}} I_{-}^{(2,1)}=\frac{\pi^{2}}{15} \\
& \bar{P}_{\gamma}=\frac{2}{6 \pi^{2}} I_{-}^{(0,3)}=\frac{\pi^{2}}{45} \\
& \bar{s}_{\gamma}=\bar{\rho}_{\gamma}+\bar{P}_{\gamma}=\frac{4 \pi^{2}}{45} .
\end{aligned}
$$

where the definitions for the $I_{ \pm}^{(p, q)}$ are given in App. A.1. Similarly for neutrinos, and assuming that they have a negligible chemical potential, the reduced variables defined in case of LTE, take the constant values

$$
\begin{aligned}
& \bar{n}_{\nu}=\frac{2}{2 \pi^{2}} I_{+}^{(1,1)}=\frac{3}{4} \bar{n}_{\gamma} \\
& \bar{\rho}_{\nu}=\frac{2}{2 \pi^{2}} I_{+}^{(2,1)}=\frac{7}{8} \bar{\rho}_{\gamma} \\
& \bar{P}_{\nu}=\frac{2}{6 \pi^{2}} I_{+}^{(0,3)}=\frac{7}{8} \bar{P}_{\gamma} \\
& \bar{s}_{\nu}=\bar{\rho}_{\nu}+\bar{P}_{\nu}=\frac{7}{8} \bar{s}_{\gamma},
\end{aligned}
$$

where we used $g_{\nu}=1$ since only left-handed neutrinos contribute to the relativistic species, but we have multiplied by a factor 2 since conventionally we add together the contributions of neutrinos and antineutrinos ${ }^{6}$. In particular we deduce from Eq. (13) that for photons or (massless) neutrinos in thermodynamical equilibrium

$$
\frac{\mathrm{d}(a T)}{\mathrm{d} t}=\frac{\mathrm{d}\left(a T_{\nu}\right)}{\mathrm{d} t}=0,
$$

if they are completely decoupled from other species. During BBN, $a T$ varies because of electron-positron annihilations. It is customary to define (Mangano et al. 2005 de Salas and Pastor, 2016)

$$
z \equiv a(T) T \quad z_{\nu} \equiv a\left(T_{\nu}\right) T_{\nu}
$$

to characterize this total variation of $a T$ and $a T_{\nu}$, with the convention that long before decoupling when all species were coupled together and at the same temperature, that is when $T=T_{\nu} \gg m_{e}$, we had $a(T) T=$ $a\left(T_{\nu}\right) T_{\nu}=1$, that is $z=z_{\nu}=1$ at early times.

\footnotetext{
${ }^{6}$ If neutrinos were Majorana particles, neutrinos would be their own antiparticle but they would possess both helicities and one would take $g=2$, resulting in the same final result
}

Additionally, in a first approximation, one can also assume that during BBN neutrinos are fully decoupled, implying that $a T_{\nu}$ is constant and $z_{\nu}=1$ remains always true. The tiny effect of incomplete decoupling induces in fact a small variation of $z_{\nu}$ that we shall take into account in $\S \amalg$ II.F. Ignoring it, the neutrino temperature scales simply as

$$
T_{\nu}=\frac{a_{0} T_{0}}{a z_{0}} \quad z_{0} \equiv z\left(T \ll m_{e}\right),
$$

where $T_{0}$ is the photons temperature today and $z_{0}$ is the value of $z$ long after BBN is finished.

\section{B. Plasma temperature}

Since for the plasma $S=s a^{3}$ is conserved, we can obtain the relation between temperature and scale factor. From Eq. 24) and expressions in App. A, we find that the ratio between plasma entropy at a given time of BBN and photons entropy today is given by

$$
\begin{aligned}
\frac{s}{s_{0}} & =\frac{T^{3}}{T_{0}^{3}} \mathcal{S}(T) \\
\mathcal{S}(T) & \equiv \frac{\bar{s}_{\mathrm{pl}}}{\bar{s}_{\gamma}}=\frac{\bar{s}_{\gamma}+\bar{s}_{e^{+}}(T)+\bar{s}_{e^{-}}(T)}{\bar{s}_{\gamma}} \\
& =1+\frac{2}{\pi^{2} \bar{s}_{\gamma}}\left[\frac{1}{3} I_{+}^{(0,3)}(x)+I_{+}^{(2,1)}(x)\right]
\end{aligned}
$$

where $x \equiv m_{e} / T$. The function $\mathcal{S}$ is plotted in Fig. 4 . For $T \gg m_{e}, \mathcal{S} \rightarrow 11 / 4$ whereas for $T \ll m_{e}, \mathcal{S} \rightarrow 1$. Entropy conservation $\left(s a^{3}=s_{0} a_{0}^{3}\right)$ allows then to relate the scale factor to the plasma temperature as

$$
\frac{z_{0}^{\text {stand }}}{z^{\text {stand }}}=\frac{a_{0} T_{0}}{a T}=\mathcal{S}^{1 / 3} \Rightarrow a(T)=\frac{a_{0} T_{0}}{T \mathcal{S}(T)^{1 / 3}} .
$$

Here $z^{\text {stand }}$ denotes $z$ in the case where we can ignored incomplete neutrino decoupling and QED plasma effects which we analyze in $\S$ II.E and II.F respectively. The inverse relation $T(a)$ is obtained by numerical inversion. $z_{0}^{\text {stand }}$ takes the value

$$
z_{0}^{\text {stand }}=S^{1 / 3}\left(T \gg m_{e}\right)=\left(\frac{11}{4}\right)^{1 / 3} \simeq 1.40102 .
$$

From Eqs. (31) and (29) the ratio between neutrino and plasma temperatures is given by

$$
\frac{T_{\nu}}{T}=\frac{\mathcal{S}^{1 / 3}(T)}{z_{0}^{\text {stand }}},
$$

and tends to $1 / z_{0}^{\text {stand }}=(4 / 11)^{1 / 3}$ at low temperatures, which is the celebrated result of instantaneous decoupling. 


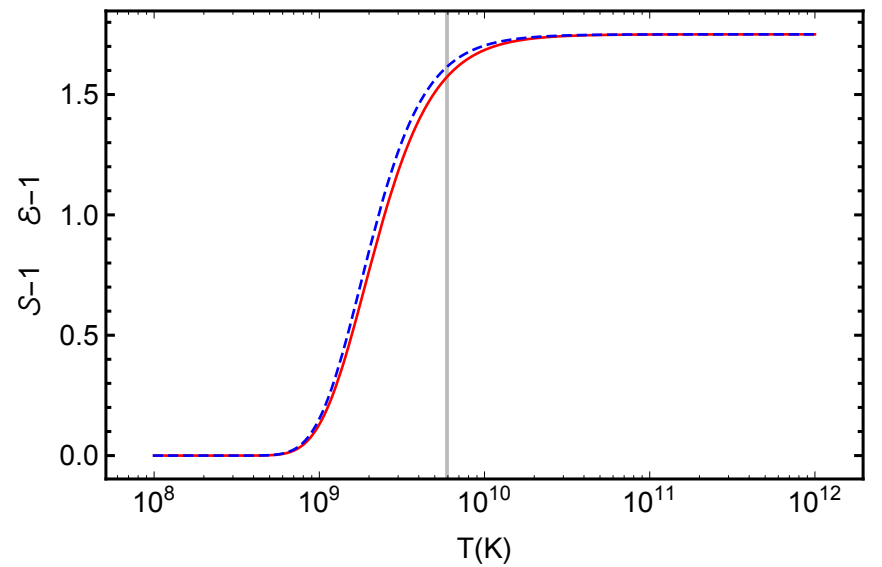

FIG. 4 Red continuous line: $\mathcal{S}(T)-1$. Blue dashed line : $\mathcal{E}(T)-1$. The vertical bar corresponds to $T=m_{e} \simeq$ $0.511 \mathrm{MeV}$.

\section{Baryon density}

In order to obtain the energy density of baryons, it is sufficient to use that for non-relativistic and cold species $\rho \propto 1 / a^{3}$. Hence

$$
\rho_{\mathrm{b}}=\left(\frac{a_{0}}{a}\right)^{3} \rho_{0}^{\text {crit }} \Omega_{\mathrm{b}}=\left(\frac{a_{0}}{a}\right)^{3} \rho_{100}^{\text {crit }} \times\left(\Omega_{\mathrm{b}} h^{2}\right)
$$

where we defined the critical densities $\rho_{0}^{\text {crit }} \equiv 3 H_{0}^{2} /(8 \pi G)$ and $\rho_{100}^{\text {crit }} \equiv 3 H_{100}^{2} /(8 \pi G)$, with the reduced Hubble rate $h \equiv H_{0} / H_{100}$ (with the standard definition $H_{100} \equiv$ $100 \mathrm{~km} / \mathrm{s} / \mathrm{Mpc}$ ). The numerical value of $\rho_{100}^{\text {crit }}$ can be found in appendix $\mathrm{D}$. The baryon energy density can be converted into a number density by estimating the average mass of nucleons

$$
n_{\mathrm{b}}=\frac{\rho_{\mathrm{b}}}{m_{\mathrm{b}}} .
$$

More details about the definition of $m_{\mathrm{b}}$ are given in App. C. We define the ratio between baryons and photons by

$$
\eta \equiv \frac{n_{\mathrm{b}}}{n_{\gamma}} .
$$

Since $n_{\mathrm{b}} \propto 1 / a^{3}$ and $n_{\gamma} \propto T^{3}$ it can be rewritten in the form

$$
\eta=\eta_{0}\left(\frac{z_{0}}{z}\right)^{3}
$$

Using Eq. (C6), the value today is approximately found to be

$$
\begin{aligned}
\eta_{0} \simeq & 6.0913 \times 10^{-10}\left(\frac{\Omega_{\mathrm{b}} h^{2}}{0.02225}\right)\left(\frac{2.7255}{T_{0}}\right)^{3} \\
& \times\left(\frac{1-1.759 \times 10^{-3}}{1-1.759 \times 10^{-3} \frac{Y_{\mathrm{P}}}{0.24709}}\right)
\end{aligned}
$$

Finally, note that we can safely ignore the thermal energy of baryons since its ratio with the energy density of photons is of order

$$
\frac{n_{\mathrm{b}} T}{\rho_{\gamma}}=\frac{\bar{n}_{\mathrm{b}}}{\bar{\rho}_{\gamma}} \propto \eta \ll 1,
$$

thus justifying the use of the scaling (34) which is the one of cold particles.

\section{Cosmology and scale factor}

The evolution of the scale factor is dictated by the Friedmann equation

$$
H^{2}=\frac{8 \pi G}{3} \rho
$$

It determines the evolution of the scale factor $a(t)$, and to solve it we need the expressions of the total energy density as a function of $a$. Since we already have found the relations $a(T)$, it is sufficient to express the energy density in terms of temperature. This is fortunate, because the energy density of the plasma depends only on temperature as it is always in local thermodynamical equilibrium. From App. A, we find that for the plasma

$$
\begin{aligned}
\rho_{\mathrm{pl}} & =\mathcal{E}(T) \bar{\rho}_{\gamma} T^{4}, \\
\mathcal{E}(T) & \equiv \frac{\bar{\rho}_{\gamma}+\bar{\rho}_{e^{+}}+\bar{\rho}_{e^{-}}}{\bar{\rho}_{\gamma}}=1+\frac{30}{\pi^{4}} I_{+}^{(2,1)}(x),
\end{aligned}
$$

where we use the definitions A5 - The function $\mathcal{E}$ is plotted in Fig. 4 and in the limit $T \gg m_{e}$ it also tends to $11 / 4$. The energy density of the $N_{\nu}$ generations of (massless) neutrinos is

$$
\rho_{\nu}=N_{\nu} \bar{\rho}_{\nu} T_{\nu}^{4}
$$

As for the energy density of baryons, we use the simple dilution relation (34) which amounts to neglecting their thermal energy. Hence a similar scaling can be used for cold dark matter.

Using that ${ }^{7}$

$$
\rho=\rho_{\nu}+\rho_{\mathrm{pl}}+\rho_{\mathrm{b}}+\rho_{\mathrm{cdm}}
$$

we obtain $\rho(T)$ that we combine with the relation $T(a)$ obtained in section II.B to get $\rho(a)$, such that the Friedmann equation 40 is in the form of an ordinary differential equation for the function $a(t)$ that we can solve numerically. The relation $t(a)$ is then deduced by numerical inversion.

\footnotetext{
7 The effect of the cosmological constant is also totally negligible as it accounts for less than $10^{-30}$ of the energy content during $\mathrm{BBN}$ even if it dominates today.
} 


\section{E. QED corrections for the plasma thermodynamics}

The thermal bath of electrons and positrons has electromagnetic interactions with the bath of photons. Using QED, this leads to a modification of the plasma thermodynamics which depend on its temperature. First this leads to an effective modification of the electron and photon masses, which come from the diagrams 5 and 6

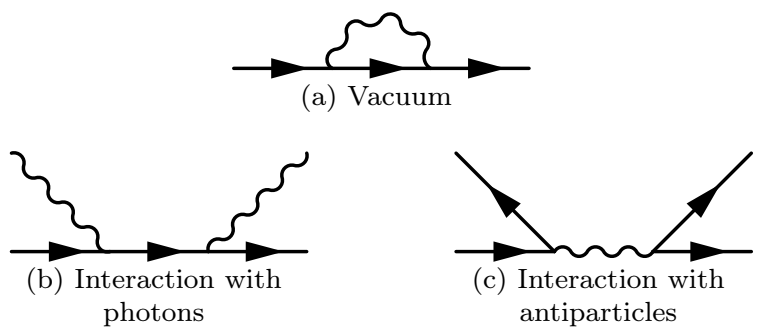

FIG. 5 Top : electron/positron self-energy. Bottom : electron/positron mass shift from interaction with plasma.

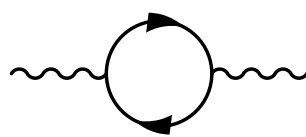

(a) Vacuum

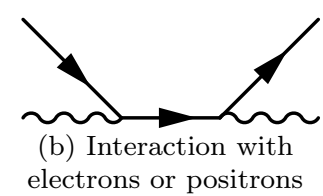

FIG. 6 Left : photon self-energy. Right : photon mass shift from interaction with electron/positron plasma.

The electron mass is shifted by (see Mangano et al. (2002, Eq. 12), Lopez and Turner (1999, Eq. 35) or Fornengo et al. (1997); Heckler (1994) for further details on its derivation)

$$
\begin{aligned}
\frac{\delta m_{e}^{2}(p, T)}{T^{2}} & =\frac{4 \alpha}{\pi}\left[I_{-}^{(0,1)}+I_{+}^{(0,1)}(x)\right] \\
& -\frac{2 \bar{m}_{e}^{2} \alpha}{\pi \bar{p}} \int_{0}^{\infty} \mathrm{d} \bar{q} \frac{\bar{q}}{\mathcal{E}_{\bar{q}}} \ln \left|\frac{\bar{p}+\bar{q}}{\bar{p}-\bar{q}}\right| g^{+}\left(\mathcal{E}_{\bar{q}}\right)
\end{aligned}
$$

where we used the definitions $\mathrm{A} 5 \mathrm{~A}-\mathrm{A} 7)$. In this expression, $\mathcal{E}_{\bar{q}} \equiv \sqrt{\bar{q}^{2}+x^{2}}, \bar{m}_{e}=x=m_{e} / T \bar{p} \equiv p / T$ and $g^{ \pm}\left(\mathcal{E}_{\bar{q}}\right) \equiv 1 /\left(\mathrm{e}^{\mathcal{E}_{\bar{q}}} \pm 1\right)$. The second contribution, which depends explicitly on the $e^{ \pm}$momentum, accounts for less than $10 \%$ to the total mass shift (see Lopez and Turner (1999); Mangano et al. (2002)). The mass shift (44) can be further simplified using

$$
I_{-}^{(0,1)} \equiv \int_{0}^{\infty} g^{-}(\bar{k}) \bar{k} \mathrm{~d} \bar{k}=\frac{\pi^{2}}{6} .
$$

The photon mass shift is instead given by

$$
\frac{\delta m_{\gamma}^{2}}{T^{2}}=\frac{8 \alpha}{\pi} I_{+}^{(0,1)}(x) .
$$

These mass shifts induce a change in the pressure of the plasma whose expression is given by Heckler (1994, Eq.
13) or Mangano et al. (2002, Eq. 16). The pressure shift reads

$$
\begin{aligned}
\overline{\delta P} \equiv \frac{\delta P}{T^{4}}= & -\int_{0}^{\infty} \frac{\mathrm{d} \bar{p}}{2 \pi^{2}} \frac{\bar{p}^{2}}{\mathcal{E}_{\bar{p}}} \frac{\delta m_{e}^{2}(p, T)}{T^{2}} g^{+}\left(\mathcal{E}_{\bar{p}}\right) \\
& -\int_{0}^{\infty} \frac{\mathrm{d} \bar{k}}{2 \pi^{2}} \frac{\bar{k}}{2} \frac{\delta m_{\gamma}^{2}(T)}{T^{2}} g^{-}(\bar{k}) .
\end{aligned}
$$

Hence from the expressions of the mass shifts we get

$$
\overline{\delta P}=\overline{\delta P}_{\mathrm{d}}+\overline{\delta P}_{\mathrm{s}},
$$

where the dominant and subdominant terms are respectively

$$
\begin{aligned}
& \overline{\delta P}_{\mathrm{d}} \equiv \frac{\alpha_{\mathrm{FS}}}{\pi}\left[-\frac{2}{3} I_{+}^{(0,1)}(x)-\frac{2}{\pi^{2}}\left(I_{+}^{(0,1)}(x)\right)^{2}\right] \\
& \overline{\delta P}_{\mathrm{s}} \equiv \frac{\alpha_{\mathrm{FS}}}{\pi^{3}} \frac{\bar{m}_{e}^{2}}{\bar{p} \bar{q}} \int \frac{\bar{p}^{2} \mathrm{~d} \bar{p}}{\mathcal{E}_{\bar{p}}} \frac{\bar{q}^{2} \mathrm{~d} \bar{q}}{\mathcal{E}_{\bar{q}}} \ln \left|\frac{\bar{p}+\bar{q}}{\bar{p}-\bar{q}}\right| g^{+}\left(\mathcal{E}_{\bar{p}}\right) g^{+}\left(\mathcal{E}_{\bar{q}}\right) .
\end{aligned}
$$

These pressure modifications are plotted in Fig. 7. We can check that the subdominant contribution is indeed negligible since QED plasma corrections are already very small (see $\S$ V.C), but we include it anyway for completeness.

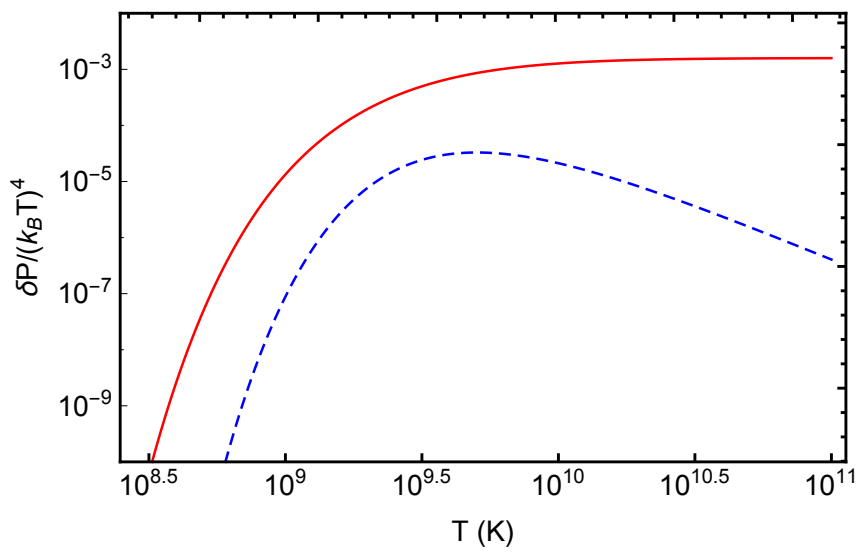

FIG. 7 Red continuous line $: \overline{\delta P}_{\mathrm{d}}$. Blue dashed line $: \overline{\delta P}_{\mathrm{s}}$. Definitions are given in Eq. 49 .

The modification of the plasma energy density is then obtained from the thermodynamic identity ${ }^{8}$

$$
\rho=-P+T \frac{\mathrm{d} P}{\mathrm{~d} T}
$$

so that we get immediately

$$
\overline{\delta \rho} \equiv \frac{\delta \rho}{T^{4}}=3 \overline{\delta P}+\frac{\partial \overline{\delta P}}{\partial \ln T} .
$$

\footnotetext{
8 Choosing $T$ and $V$ to describe the state of a system, then $P$ and $\rho$ are functions of $T$ only since they are intensive quantities. It follows from $T \mathrm{~d} S=\mathrm{d}(\rho V)+P \mathrm{~d} V$ that $\mathrm{d} S=(P+\rho) / T \mathrm{~d} V+$ $V / T(\mathrm{~d} \rho / \mathrm{d} T) \mathrm{d} T$. From the integrability conditions we then get $\partial_{T}[(\rho+P) / T]=\partial_{V}(V / T \mathrm{~d} \rho / \mathrm{d} T)$ and the identity 50 follows.
} 
Lopez and Turner (1999) defined corrected relativistic degrees of freedom by

$$
\delta g_{\rho} \equiv 2 \frac{\overline{\delta \rho}}{\bar{\rho}_{\gamma}}=\frac{30}{\pi^{2}} \overline{\delta \rho}, \quad \delta g_{P} \equiv 2 \frac{\overline{\delta P}}{\bar{P}_{\gamma}}=\frac{90}{\pi^{2}} \overline{\delta P} .
$$

At high temperatures, that is for $T \gg m_{e}$, we obtain us$\operatorname{ing} I_{+}^{(0,1)}(x=0)=\pi^{2} / 12$ (see table IX in appendix A.1 the limits

$$
\overline{\delta \rho} \simeq-\frac{5}{96}(4 \pi \alpha), \quad \overline{\delta P} \simeq \frac{1}{3} \overline{\delta \rho},
$$

which expressed in terms of corrected relativistic degrees of freedom read

$$
\delta g_{\rho} \simeq \delta g_{P} \simeq-\frac{25 \alpha}{4 \pi} \simeq 0.01452 .
$$

These corrections are plotted in Fig. 8 together with the high temperature limit.

The QED plasma corrections enter in four places.

- First they imply that the entropy of the plasma as given in Eq. $30 \mathrm{~b}$ must be modified and we must use

$$
\mathcal{S}^{\mathrm{QED}}=\mathcal{S}+\frac{\bar{\rho}_{\gamma}}{\bar{s}_{\gamma}} \frac{\delta g_{\rho}}{2}+\frac{\bar{P}_{\gamma}}{\bar{s}_{\gamma}} \frac{\delta g_{P}}{2}=\mathcal{S}+\frac{\delta g_{P}+3 \delta g_{\rho}}{8}
$$

where the last equality follows from Eqs. (25). $\mathcal{S}^{\mathrm{QED}}$ must be used instead of $\mathcal{S}$ in Eq. 31] in order to obtain $a(T)$.

- Second, this entropy modification also affects the evolution of $z$ and we find

$$
\left(z_{0}^{\mathrm{QED}}\right)^{3}=S^{\mathrm{QED}}\left(T \gg m_{e}\right)=\frac{11}{4}\left(1-\frac{4}{11} \frac{25 \alpha}{8 \pi}\right),
$$

that is $z_{0}^{\mathrm{QED}} \simeq 1.39979$. This affects the neutrino temperature scaling which then becomes

$$
T_{\nu}^{\mathrm{QED}}=\frac{a_{0} T_{0}}{a z_{0}^{\mathrm{QED}}} .
$$

This neutrino temperature must be used in Eq. 42 when computing the energy density of neutrinos for the Friedmann equation (40).

- Third, the plasma QED correction must also be incorporated in the energy density of the plasma when solving the Friedmann equation 40, meaning that we must modify Eqs. 41 and use instead

$$
\mathcal{E}^{\mathrm{QED}}=\mathcal{E}+\frac{\delta g_{\rho}}{2}
$$

when computing $\rho_{\mathrm{pl}}$ for the Friedmann equation 40.

- Finally, the electron mass shifts modifies the statistics of electrons and positrons in the weakinteractions.
An important comment is in order here on the last effect. Since the mass shift (44) is exactly Eq. (5.12) of (Brown and Sawyer, 2001) (that is $2 E \Delta E=\delta m_{e}^{2}$ ), the effect of the electron mass shift is one of the several effects involved in finite-temperature radiative corrections for the weak rates. Hence, following Brown and Sawyer (2001), we consider that the modification of the statistics through the mass shift is part of the finite-temperature corrections and we do not consider that it is part of the QED plasma corrections. This point of view is similar to Esposito et al. (2000a); Serpico et al. (2004) but different from Lopez and Turner (1999). Hence comparisons on the magnitude of the correction must take into account the point of view on the effect of mass shifts, since either they are reported as QED effects or finite-temperature radiative corrections.

It turns out that the QED plasma corrections (that is without the effect of the mass shift on the weak-interaction rates), coming from the modifications 55), 57) and (58), are very small since they modify the Helium production by approximately $\Delta Y_{\mathrm{P}}^{(4)}=-0.1$ (see $\S$ V.C.

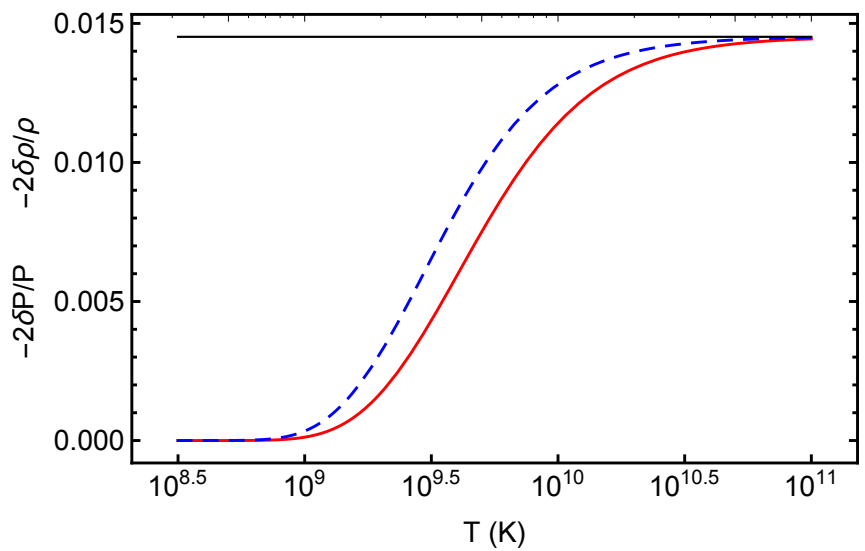

FIG. 8 Effective degrees of freedom in the plasma from QED corrections. Red continuous line : $-\delta g_{P}=-2 \delta P / P$. Blue dashed line : $-\delta g_{\rho}=-2 \delta \rho / \rho$. Black thin line : High temperature asymptotic value $25 \alpha /(4 \pi)$.

\section{F. Incomplete neutrino decoupling}

The effect of incomplete neutrino decoupling has been studied in details in Birrell et al. (2014); Dodelson and Turner (1992); Dolgov et al. (1997, 1999); Esposito et al. (2000b); Fields et al. (1993); Gnedin and Gnedin (1998); Grohs et al. (2016); Hannestad (2002); Hannestad and Madsen (1995); Mangano et al. (2002, 2005, 2006); de Salas and Pastor (2016). Electron-positron annihilations lead to a small reheating of the neutrino bath which must be studied in the context of coupled Boltzmann equations since it also leads to spectral distortions in the neutrino spectrum. Furthermore the complete study, 
that we do not reproduce here, requires to consider neutrino flavor oscillations.

Let us first study the effect of incomplete neutrino decoupling (ID) on the plasma. If there is heat exchange, that is energy exchange between the plasma and the neutrinos, then the evolution of the plasma entropy is dictated by Eq. 20) with the chemical potential neglected, that is

$$
\dot{S}_{\mathrm{pl}}=\frac{\dot{Q}_{\mathrm{pl}}}{T} .
$$

This is always true for the plasma because it is always at local thermodynamic equilibrium, but not necessarily true for the neutrinos for which we should rely on Eq. 177) if we were to compute the evolution of their entropy. The numerical studies of the effect of incomplete neutrino decoupling gives a fit to the heating rate of the plasma in Pisanti et al. (2008). More precisely, we define the dimensionless function $\mathcal{N}(T)$ related to the heating rate as

$$
\frac{\dot{q}_{\mathrm{pl}}}{H T}=-T^{3} \mathcal{N}(T),
$$

where we remind $\dot{Q}=a^{3} \dot{q}$. The function $T^{4} \mathcal{N}$ can be viewed as the volume heating rate in units of the Hubble rate $H$. The fit given in Pisanti et al. (2008, Eqs. A24A25) is a 13-order polynomial in $x=m_{e} / T$ valid for $x<4$, and we plot it in Fig. 9

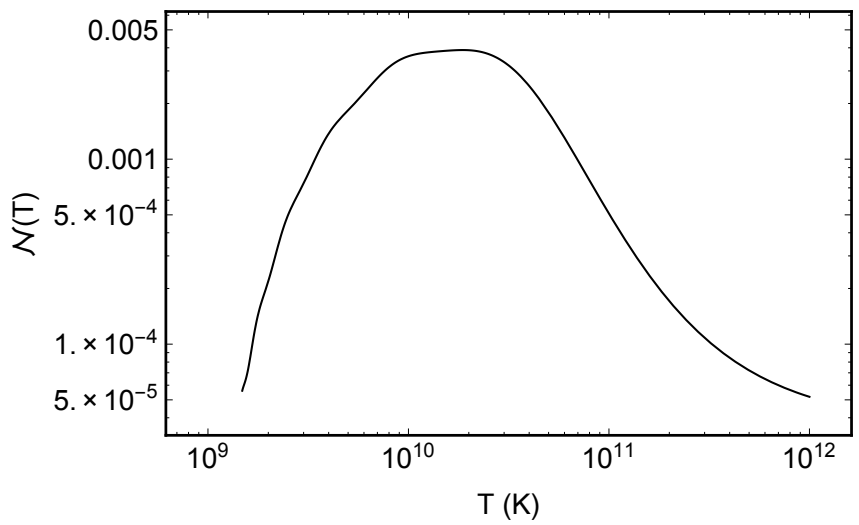

FIG. $9 \mathcal{N}(T)$ as defined in Eq. 60.

From Eqs. (59) and $(60)$, the evolution of the plasma reduced entropy is dictated by

$$
\frac{1}{(a T)^{3}} \frac{\mathrm{d}\left[\bar{s}_{\mathrm{pl}}(a T)^{3}\right]}{\mathrm{d} \ln a}=-\mathcal{N} .
$$

From the definition $30 \mathrm{~b}$ we obtain

$$
\frac{\mathrm{d} \ln (a T)}{\mathrm{d} \ln T}=\frac{\mathcal{N}-\frac{\mathrm{d} \mathcal{S}}{\mathrm{d} \ln T}}{\mathcal{N}+3 \mathcal{S}}
$$

where $\mathcal{S}$ must be replaced by $\mathcal{S}^{\mathrm{QED}}$ when including plasma QED effects. We recover when $\mathcal{N}=0$, that is for complete neutrino decoupling, that $z=a T \propto \mathcal{S}^{-1 / 3}$. This equation can be integrated numerically to obtain $a(T)$ in the case of incomplete neutrino decoupling, and a numerical inversion allows to obtain subsequently $T(a)$. $z$ is modified because of the non-conservation of plasma entropy, and we get $z_{0}^{\mathrm{ID}} \simeq 1.39911$ if QED plasma effects are not included and $z_{0}^{\mathrm{ID}, \mathrm{QED}} \simeq 1.39788$ if they are also included, in agreement (except for the last digit) with table 1 of de Salas and Pastor (2016). Our results are summarized in table $\llbracket$

Let us now focus on the effect of incomplete neutrino decoupling on neutrinos. Since the energy taken from the plasma is gained by the neutrinos, we have necessarily $q_{\mathrm{pl}}=-q_{\nu}$. Hence we can solve for the evolution of the neutrino energy density from Eq. (12) which is

$$
\frac{1}{a^{4}} \frac{\mathrm{d}\left(a^{4} \rho_{\nu}\right)}{\mathrm{d} \ln a}=-\frac{\dot{q}_{\mathrm{pl}}}{H}=T^{4} \mathcal{N} .
$$

Since $T^{4} \mathcal{N}$ is a function of $T$ it can be considered as a function of $a$ using the $T(a)$ previously obtained. Solving numerically this differential equation gives $\rho_{\nu}(a)$. Together with the previously solved $T(a)$ [which allows to get $\rho_{\mathrm{pl}}(a)$ from Eq. [41]] and the QED correction (58), we obtain $\rho(a)$ and thus the relation $a(t)$ and its inverse $t(a)$ from numerically solving the Friedmann equation $(40)$.

This approach to compute the variation of the neutrino energy density from Eq. 63) is correct even if incomplete neutrino decoupling and neutrino reheating by electronpositron annihilations create spectral distortions in the neutrino spectra. That is, the gravitational effect of incomplete neutrino decoupling is taken into account correctly with our approach. However it cannot fully take into account the effect on the weak rates which we discuss further in $\S$ III.I and V.C.5.

\section{G. Effective description of neutrinos}

Neutrinos are not in local thermodynamical equilibrium and their temperature is not well defined. Indeed, the full characterization of the neutrinos requires to solve the coupled Boltzmann equations dictating the evolution of neutrino distribution functions, and we must in principle build a temperature definition from the energy spectrum (see e.g. Pitrou and Stebbins (2014)). In the BBN context it is convenient to define a brightness temperature, being the temperature of the Fermi-Dirac distribution with no chemical potential that would have the same energy density. Hence we define $T_{\nu}$ as in Eq. (42) by

$$
\rho_{\nu}=N_{\nu} \bar{\rho}_{\nu} T_{\nu}^{4} \quad \Rightarrow \quad \rho_{\nu} a^{4}=N_{\nu} \bar{\rho}_{\nu} z_{\nu}^{4},
$$

where $\rho_{\nu}$ results from the integration of Eq. 63 ..$z_{\nu}{ }^{9}$ is deduced from the value of $\rho_{\nu} a^{4}$ obtained numerically,

\footnotetext{
${ }^{9}$ Note that we still define $z_{\nu}$ as in the LTE case [Eq. 28p].
} 
and we find $z_{\nu, 0}=1.00144$. It corresponds to a neutrino energy density increase of a factor $z_{\nu, 0}^{4}=1.00576$ compared to the complete decoupling scenario.

The ratio between neutrino and photon temperature is simply

$$
\frac{T_{\nu}}{T}=\frac{z_{\nu}}{z}
$$

Using this ratio, Eq. (64) can be rewritten to define an effective number of neutrinos as

$$
\rho_{\nu}=N_{\text {eff }} \bar{\rho}_{\nu}\left(\frac{T}{z^{\text {stand }}}\right)^{4} \quad N_{\text {eff }} \equiv N_{\nu}\left(\frac{z_{\nu} z^{\text {stand }}}{z}\right)^{4} .
$$

This is the number of neutrinos that would be required to have the same energy density if the ratio $T_{\nu} / T$ was taken from the complete decoupling result without QED correction, that is $1 / z^{\text {stand }}$. $N_{\text {eff }}$ today is completely determined by the values of $z_{0}$ and $z_{\nu, 0}$. Its evolution is plotted in Fig. 10.

We find $N_{\mathrm{eff}}^{\mathrm{ID}}=3.0337$ today if QED plasma effects are ignored $\left[z\right.$ obtained from Eq. 62 and $z_{\nu}$ from Eqs. (63) and (65) but non-instantaneous decoupling is taken into account. We find $N_{\text {eff }}^{\mathrm{QED}}=3.0106$ for the reverse situation $\left(z_{\nu}=1\right.$ but $\left.z=z^{\mathrm{QED}}\right)$. If both effects are taken into account $\left[z\right.$ obtained from Eq. $\sqrt{62}$ with $\mathcal{S}^{\mathrm{QED}}$ instead of $\mathcal{S}$, and $z_{\nu}$ from Eqs. 63) and [65]], we find $N_{\mathrm{eff}}^{\mathrm{ID}, \mathrm{QED}}=3.0444$, in very close agreement with the last results of de Salas and Pastor (2016).

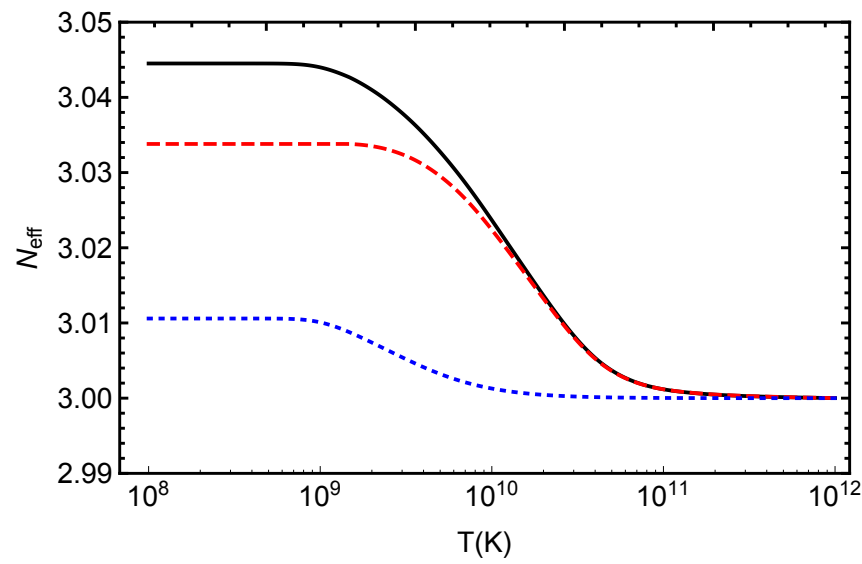

FIG. $10 N_{\text {eff }}(T)$. Black continuous line: both QED plasma effects and incomplete neutrino decoupling. Red long dashes: Incomplete decoupling but no QED corrections. Blue small dashes: QED correction with complete decoupling

After BBN, e.g. for the evolution of initial perturbations through baryon acoustic oscillations, only the energy density of neutrinos is important through its gravitational effect. Distortions in the neutrino spectra can have in principle an effect on CMB and structure formation when considering its joint effect with neutrino masses at late times. However this effect is expected to be extremely small and it is enough to assume that after
TABLE I $z_{0}, z_{\nu, 0}$ and $N_{\text {eff }}$ depending on the effects.

\begin{tabular}{ccccc}
\hline QED & Decoupling & $z_{0}=a T$ & $z_{\nu, 0}=a T_{\nu}$ & $N_{\text {eff }}$ \\
\hline No & Yes & 1.40102 & 1.00000 & 3.0000 \\
Yes & Yes & 1.39979 & 1.00000 & 3.0106 \\
No & No & 1.39911 & 1.00144 & 3.0338 \\
Yes & No & 1.39788 & 1.00144 & 3.0445 \\
\hline
\end{tabular}

BBN neutrinos follow a Fermi-Dirac distribution. Hence $N_{\text {eff }}$ is used in CMB codes such as CAMB (Lewis and Challinor, 1999 Lewis et al., 2000) and CLASS (Blas et al. 2011; Lesgourgues, 2011) to take into account the effect of incomplete decoupling during BBN. From the definition (66) the total energy density of radiation after $\mathrm{BBN}$ is

$$
\rho_{\mathrm{R}}=\rho_{\nu}+\rho_{\gamma}=\rho_{\gamma}\left(1+\frac{7}{8} N_{\mathrm{eff}}\left(\frac{4}{11}\right)^{4 / 3}\right)
$$

meaning that we can forget about the QED plasma corrections and the incomplete neutrino decoupling during BBN if we use $N_{\text {eff }}$ instead of $N_{\nu}=3$.

The values of $z_{0}, z_{\nu, 0}$ and $N_{\text {eff }}$ for the various cases are reported in table II Let us comment on the value $N_{\text {eff }} \simeq 3.02$ found in Grohs et al. (2016) for the effect of QED alone. It is important to realize that we must be careful not to replace the mass shifts (44) and (46) directly in the distribution function inside the general expression (A2c) for pressure to obtain the pressure shift (47), since this would overestimate it by a factor 2 . This subtlety is detailed in Heckler (1994) and is correctly taken into account in Mangano et al. (2002). Since this is what has been done in Grohs et al. (2016), it explains why the effect of QED on the plasma as measured by $N_{\text {eff }}$ found in this reference is around 3.02 instead of 3.01.

\section{WEAK INTERACTIONS}

Once the dynamics of the background has been solved, it is possible to study the evolution of the abundance of neutrons. This determines the final amount of chemical species since atomic nuclei form from the fusion of neutrons and protons. Throughout we assume that all particles are in local thermodynamical equilibrium. If this is certainly true for the plasma of photons strongly coupled with electrons and positrons, this is not exactly true for neutrinos. Indeed, as we have seen in $\S$ II.F. neutrinos are not fully decoupled when BBN takes place and there is a residual heating of neutrinos which cannot be fully described as an increased neutrino temperature, since it also leads to a distorted neutrino spectrum. 


\section{A. General formulation}

The weak interaction reactions correspond to a set of reactions which are all related by crossing symmetry. These are the six reactions

$$
\begin{aligned}
n+\nu & \leftrightarrow p+e^{-} \\
n & \leftrightarrow p+e^{-}+\bar{\nu} \\
n+e^{+} & \leftrightarrow p+\bar{\nu}
\end{aligned}
$$

where $n(p)$ stands for neutrons (protons). The two reactions $n+e^{+}+\nu \leftrightarrow p$ are not possible energetically since $m_{n}>m_{p}$, as can be seen considering the reaction in the proton rest mass frame. However it can exist if there is a photon in the final state of the forward reaction, see appendix $\S$ B.7.

It follows that the number density of neutrons and protons is not just diluted by expansion, but varies according to the reaction rates. The general form for the neutronproton density evolution is

$$
\begin{array}{r}
\dot{n}_{n}+3 H n_{n}=-n_{n} \Gamma_{n \rightarrow p}+n_{p} \Gamma_{p \rightarrow n} \\
\dot{n}_{p}+3 H n_{p}=-n_{p} \Gamma_{p \rightarrow n}+n_{n} \Gamma_{n \rightarrow p}
\end{array}
$$

where the reaction rates $\Gamma$ are associated to the corresponding collision terms in the Boltzmann equation. Here we have gathered the rates according to

$$
\begin{aligned}
& \Gamma_{n \rightarrow p}=\Gamma_{n+\nu \rightarrow p+e}+\Gamma_{n \rightarrow p+e+\bar{\nu}}+\Gamma_{n+e \rightarrow p+\bar{\nu}} \\
& \Gamma_{p \rightarrow n}=\Gamma_{p+e \rightarrow n+\nu}+\Gamma_{p+e+\bar{\nu} \rightarrow n}+\Gamma_{p+\bar{\nu} \rightarrow n+e}
\end{aligned}
$$

The general expression for these reaction rates is detailed in App. B. For all rates with the nucleon $a$ in the initial state and nucleon $b$ in the final state, they take the form

$$
n_{a} \Gamma_{a \rightarrow b}=\int \frac{\mathrm{d}^{3} \mathbf{p}_{a} \mathrm{~d}^{3} \mathbf{p}_{e} \mathrm{~d}^{3} \mathbf{p}_{\nu}}{2^{4}(2 \pi)^{8}} \delta\left(E_{a}-E_{b}+\alpha_{e} E_{e}+\alpha_{\nu} E_{\nu}\right) \frac{|M|_{a \rightarrow b}^{2}}{E_{n} E_{p} E_{e} E_{\nu}} f_{a}\left(E_{a}\right) f_{\nu}\left(\alpha_{\nu} E_{\nu}\right) f_{e}\left(\alpha_{e} E_{e}\right)
$$

where $\alpha_{\nu}=1$ if the neutrino is in the initial state and $\alpha_{\nu}=-1$ if it is in the final state, and a similar definition for $\alpha_{e}$ according to the $\mathrm{e}^{ \pm}$position. These coefficients appear obviously in the Dirac delta function ensuring energy conservation in reactions, and they are also used to express the final nucleon momentum as $p_{b}=p_{a}+\alpha_{\nu} p_{\nu}+\alpha_{e} p_{e}$. They also appear in the distribution functions of the electrons and neutrinos, because either the particle is in the initial state and the corresponding distribution function appears in the expression, or it is in the final state and it becomes a Pauli-blocking factor thanks to the relation

$$
f(-E)=1-f(E),
$$

valid for a Fermi-Dirac distribution with vanishing chemical potential. For a given reaction, $|M|_{a \rightarrow b}^{2}$ is the corresponding matrix-element of the weak interaction summed over all initial and final states. For weak interactions in the Fermi theory, it is of the form (Fidler and Pitrou, 2017)

$$
\frac{|M|^{2}}{2^{7} G_{F}^{2}}=c_{L L} \mathcal{M}_{L L}+c_{R R} \mathcal{M}_{R R}+c_{L R} \mathcal{M}_{L R}
$$

with the coupling factors $c$. given in Eqs. B11. The expressions for $\mathcal{M}_{L L}, \mathcal{M}_{R R}, \mathcal{M}_{L R}$ are reported in Eqs. (B12). The $L L$ term (resp. $R R$ ) corresponds to purely left-chiral (resp. right-chiral) couplings, and the $L R$ term is an interference term.

\section{B. Infinite nucleon mass approximation}

Let us define the energy gap

$$
\Delta \equiv m_{n}-m_{p} \simeq 1.29333 \mathrm{MeV} .
$$

Throughout we use $g(E)$ for the Fermi-Dirac distribution at temperature of electrons $T$ and $g_{\nu}(E)$ for the FermiDirac distribution at the neutrino temperature $T_{\nu}$.

$$
g(E) \equiv \frac{1}{\left(\mathrm{e}^{\frac{E}{T}}+1\right)} \quad g_{\nu}(E) \equiv \frac{1}{\left(\mathrm{e}^{\frac{E}{T_{\nu}}}+1\right)} .
$$

In $\S$ II details are given on how these temperatures can be computed in function of the scale factor $a$ and the time $t$.

In the infinite nucleon mass limit (but keeping $\Delta$ constant), also called the Born approximation, the reaction rates take simple forms (see App. B). First, the factors entering the matrix element (73) are in that limit simply

$$
\frac{\mathcal{M}_{L L}}{E_{n} E_{p} E_{\nu} E_{e}}=\frac{\mathcal{M}_{R R}}{E_{n} E_{p} E_{\nu} E_{e}}=\frac{\mathcal{M}_{L R}}{E_{n} E_{p} E_{\nu} E_{e}}=1 .
$$

The last equality is correct only if it is understood that an angular average either on electrons momentum or neutrino momentum is performed ${ }^{10}$ (see the detailed explanation at the end of $\S$ B.2). Hence from Eq. (71), we

\footnotetext{
10 The LR coupling is at the origin of the asymmetry $(\mathrm{B} 22 \mathrm{~b})$ in the decay product of neutron beta decay (Ivanov et al., 2013 ) from which the value of $g_{A}$ is inferred.
} 
find the Born rates (Bernstein et al., 1989 ; Brown and Sawyer, 2001, Lopez and Turner, 1999: Weinberg, 1972)

$$
\begin{aligned}
\bar{\Gamma}_{n \rightarrow p} & =\bar{\Gamma}_{n \rightarrow p+e}+\bar{\Gamma}_{n+e \rightarrow p} \\
& =K \int_{0}^{\infty} p^{2} \mathrm{~d} p\left[\chi_{+}(E)+\chi_{+}(-E)\right],
\end{aligned}
$$

with $E=\sqrt{p^{2}+m_{e}^{2}}$ and

$$
\begin{aligned}
\chi_{ \pm}(E) & \equiv\left(E_{\nu}^{\mp}\right)^{2} g_{\nu}\left(E_{\nu}^{\mp}\right) g(-E), \\
E_{\nu}^{\mp} & \equiv E \mp \Delta, \\
K & \equiv \frac{4 G_{W}^{2}\left(1+3 g_{A}^{2}\right)}{(2 \pi)^{3}} .
\end{aligned}
$$

The first contribution in Eq. (77) corresponds to the $n \rightarrow p$ processes (68a) and (68b) added, that is for all processes where the electron is in the final state. It can be checked indeed that the electron distribution is evaluated as $g(-E)=1-g(E)$. Furthermore, if the neutrino is in the initial state (when $E>\Delta$ ) its energy is $E_{\nu}=E-\Delta$ and its distribution function appears as $g_{\nu}\left(E_{\nu}\right)$, but if it is in the final state (when $E<\Delta$ ) its energy is $E_{\nu}=\Delta-E$ and the neutrino distribution function is evaluated as $g_{\nu}(E-\Delta)=1-g_{\nu}(\Delta-E)$.

The second term of Eq. (77) corresponds to the reaction (68c), that is to the process where the positron is in the initial state. The energy of the positron is $E$ and its distribution function appears as an initial state $[g(E)]$, whereas the neutrinos in the final state have energy $E_{\nu}=\Delta+E$ and their distribution function appear thus as Pauli-blocking factor $g_{\nu}(-E-\Delta)=1-g_{\nu}(E+\Delta)$.

The reaction rate for protons, that is $\Gamma_{p \rightarrow n}$, is obtained by the simple replacement $\Delta \rightarrow-\Delta$, which amounts to $\chi_{+} \rightarrow \chi_{-}$. We give it for completeness

$$
\begin{aligned}
\bar{\Gamma}_{p \rightarrow n} & =\bar{\Gamma}_{p \rightarrow n+e}+\bar{\Gamma}_{p+e \rightarrow n} \\
& =K \int_{0}^{\infty} p^{2} \mathrm{~d} p\left[\chi_{-}(E)+\chi_{-}(-E)\right] .
\end{aligned}
$$

Similarly the second term corresponds to the reverse processes (68a) and (68b) added since the electron distribution function is always in an initial state $[g(E)]$, and the neutrino is in the initial or final state depending on the sign of $E_{\nu}=-E+\Delta$. The first term corresponds to the reverse process $(68 \mathrm{c})$ with the positron always in the final state $[g(-E)=1-g(E)]$ and the neutrino always in the initial state $\left[g_{\nu}(E+\Delta)\right]$.

Finally, note that using

$$
g(-E)=1-g(E)=\mathrm{e}^{E / T} g(E),
$$

we get in the case of thermal equilibrium between neutrinos and the plasma (that is when $T_{\nu}=T$ )

$$
\chi_{+}(E)=\mathrm{e}^{\Delta / T} \chi_{-}(-E) .
$$

This implies that if neutrinos have the same temperature as the plasma, the reaction rates satisfy the Born approximation detailed balance relation

$$
\bar{\Gamma}_{p \rightarrow n}=\mathrm{e}^{-\Delta / T} \bar{\Gamma}_{n \rightarrow p} .
$$

It is important that detailed balance be satisfied in our estimation of the weak rates since it is at the origin of the enforcement of the thermodynamical equilibrium between neutrons and protons. When adding corrections to the Born approximation weak rates in the form $\Gamma \equiv$ $\delta \Gamma+\bar{\Gamma}$, then the relative corrections of the forward and reverse rates must be equal, that is

$$
\frac{\delta \Gamma_{p \rightarrow n}}{\bar{\Gamma}_{p \rightarrow n}}=\frac{\delta \Gamma_{n \rightarrow p}}{\bar{\Gamma}_{n \rightarrow p}},
$$

in order that the corrected rates also satisfy the detailed balance property (86). When estimating the corrections to the Born rates, we systematically discuss how this detailed balance is kept valid with the corrections and we highlight our differences on that crucial property with previous literature.

\section{Calibration from free neutron decay rate}

The interaction rates are proportional to the factor $K$ defined in Eq. (81). It is proportional to

$$
G_{W}^{2} \equiv G_{F}^{2} \cos ^{2}\left(\theta_{\mathrm{C}}\right)
$$

where $G_{F}$ is the Fermi constant and $\theta_{\mathrm{C}}$ is a CKM angle, and also to $1+3 g_{A}^{2}$ where $g_{A}$ is the axial current constant for the nucleons (see App. D for numerical values). Given the uncertainty in these parameters, it is more precise to obtain $K$ from the free neutron decay ${ }^{11}$. Indeed, at low temperatures, $\Gamma_{n \rightarrow p}$ should be equal to the free neutron decay rate $1 / \tau_{n}$. The low temperature corresponds to (77) restricted to $E_{\nu}<0$, that is

$$
\frac{1}{\tau_{n}}=\Gamma_{n \rightarrow p}(T=0)=K \int_{0}^{\sqrt{\Delta^{2}-m_{e}^{2}}} p^{2} E_{\nu}^{2} \mathrm{~d} p,
$$

with $E_{\nu}=\sqrt{p^{2}+m_{e}^{2}}-\Delta$. We define

$$
\lambda_{0} \equiv m_{e}^{-5} K^{-1} \Gamma_{n \rightarrow p}(T=0)
$$

so $K$ is obtained from the free neutron decay rate as

$$
K=1 /\left(\tau_{n} \lambda_{0} m_{e}^{5}\right)
$$

instead of Eq. 817. At the Born approximation level, that is using $\bar{\Gamma}_{n \rightarrow p}$ in Eq. 900, $\lambda_{0}$ takes the value (Bern-

11 The relative precision on $\cos \left(\theta_{\mathrm{C}}\right)$ and $g_{A}$ are respectively $2.0 \times$ $10^{-4}$ and $1.8 \times 10^{-3}$. If $\tau_{n}$ was to be obtained theoretically from these constants, then its uncertainty $\delta \tau_{n} / \tau_{n} \simeq$ $2 \delta \cos \left(\theta_{\mathrm{C}}\right) / \cos \left(\theta_{\mathrm{C}}\right)+1.66 \delta g_{A} / g_{A}$ would be of order $3 \times 10^{-3}$ which is larger than the experimental uncertainty which is of order $1.0 \times 10^{-3}$. However improvements in the measurements of $g_{A}$ by a factor 3 would make this direct estimation of $\tau_{n}$ competitive. 
stein et al., 1989)

$$
\begin{aligned}
\bar{\lambda}_{0} & \equiv m_{e}^{-5} \int_{0}^{\sqrt{\Delta^{2}-m_{e}^{2}}} p^{2} E_{\nu}^{2} \mathrm{~d} p \\
& =\sqrt{\bar{\Delta}^{2}-1}\left(\frac{-8-9 \bar{\Delta}^{2}+2 \bar{\Delta}^{4}}{60}\right)+\frac{\bar{\Delta}}{4} \operatorname{arccosh} \bar{\Delta} \\
& \simeq 1.63609
\end{aligned}
$$

where $\bar{\Delta} \equiv \Delta / m_{e}$.

\section{Neutron abundance and freeze-out}

It is enough to consider the weak rates in the Born approximation to estimate the freeze-out temperature. The Born rates are plotted in Fig. 11 together with the Hubble rate. A first estimation of the freeze-out temperature

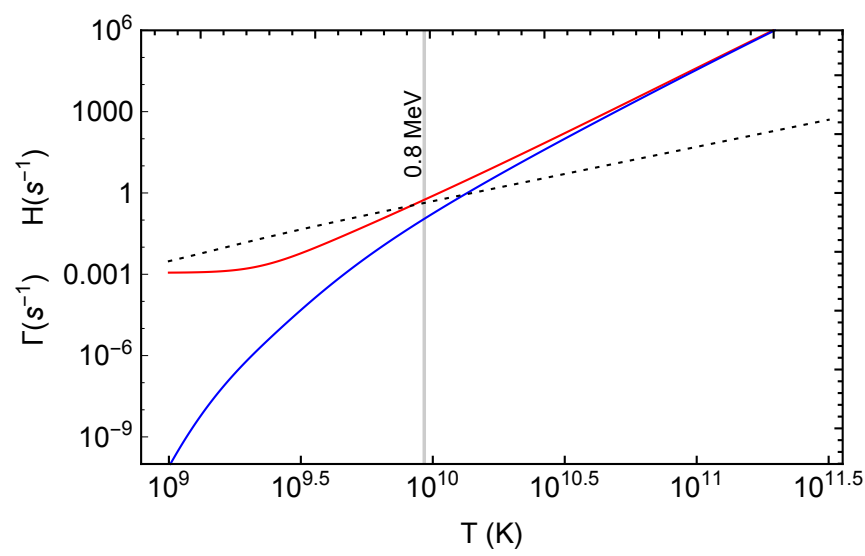

FIG. $11 \Gamma$. Continuous line: Upper red curve is $n \rightarrow p$ rate and lower blue curve is $p \rightarrow n$ rate. Dashed line: Hubble rate.

consists in noting that expansion overcomes both rates for $T_{F} \simeq 8 \times 10^{9} \mathrm{~K}$ which we can take as the freeze-out temperature.

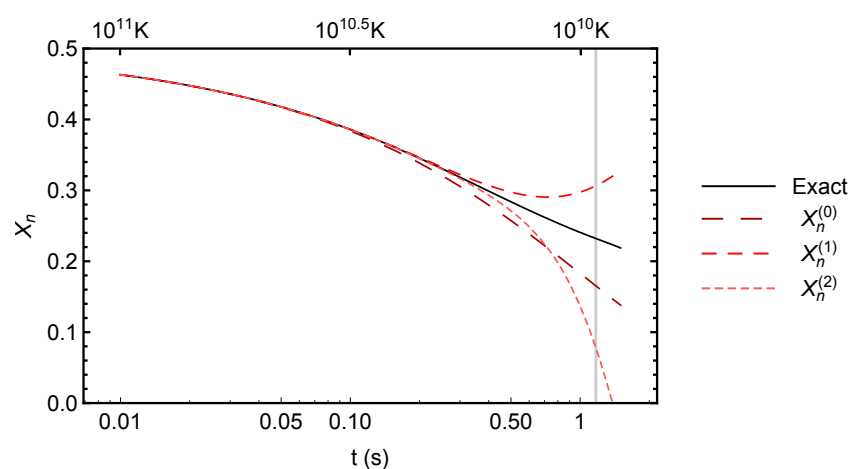

FIG. 12 Black continuous line: numerical solution. red dashed lines : solutions (95) for $p=0,1,2$. The vertical bar corresponds to $T_{F}=0.8 \mathrm{MeV}$

We can justify this estimation by finding an approximate solution for the neutron abundance. Its evolution, in absence of nuclear reactions, is dictated by

$$
\dot{X}_{n}=X_{p} \Gamma_{p \rightarrow n}-X_{n} \Gamma_{n \rightarrow p} .
$$

Using $X_{p}=1-X_{n}$, we get

$$
X_{n}=\frac{\Gamma_{p \rightarrow n}}{\Lambda}-\frac{1}{\Lambda} \dot{X}_{n}, \quad \Lambda \equiv \Gamma_{p \rightarrow n}+\Gamma_{n \rightarrow p} .
$$

We obtain tight-coupling solutions to order $m$ by recursive replacement of $X_{n}$ and we get

$$
X_{n}^{(m)}=\sum_{k=0}^{m}\left(\frac{-1}{\Lambda} \frac{\mathrm{d}}{\mathrm{d} t}\right)^{k} \frac{\Gamma_{p \rightarrow n}}{\Lambda} .
$$

$m=0$ corresponds to the pure thermodynamical equilibrium and higher order corrections are due to the fact that the neutron abundance has less and less time to approach this equilibrium value. This expansion is equivalent to Bernstein et al. (1989, Eq. 2.8). We can estimate the freeze-out temperature, that is the temperature at which weak interactions fail to maintain the thermodynamical equilibrium value, by computing the temperature for which the first correction is of order of the equilibrium value, that is when $\left|X_{n}^{(1)}-X_{n}^{(0)}\right|=X_{n}^{(0)}$. Assuming that the neutrino temperature does not differ significantly from the plasma temperature before freeze-out, we can use Eq. (86) to estimate this condition which reads

$$
H \frac{Q}{T} \frac{\mathrm{e}^{Q / T}}{1+\mathrm{e}^{Q / T}}=\Lambda
$$

We find $T_{F} \simeq 8.9 \times 10^{9} \mathrm{~K} \simeq 0.77 \mathrm{MeV}$ and replacing in the thermodynamical equilibrium abundance we get

$$
X_{n}^{F} \equiv X_{n}^{(0)}\left(T_{F}\right) \simeq 0.156 .
$$

Finally, another estimation consists in determining visually when the neutron abundance is only affected by beta decay. In Fig. 3 this leads to $T_{F} \simeq 3.3 \times 10^{9} \mathrm{~K} \simeq$ $0.28 \mathrm{MeV}$ and $X_{n}^{F} \simeq 0.17$.

After the freeze-out, the neutron abundance is only affected by the neutron beta decay. The nucleosynthesis starts approximately when $T<T_{\mathrm{Nuc}}=0.078 \mathrm{MeV}$, or 0.9 GK (see $\S \mathrm{V} . \mathrm{B}$ corresponding to roughly $t_{\mathrm{Nuc}}=$ 200 s. The neutron abundance when the nucleosynthesis starts is thus approximately given by

$$
X_{n}\left(T_{\mathrm{Nuc}}\right)=X_{n}^{F} \exp \left(-\frac{t_{\mathrm{nuc}}-t_{F}}{\tau_{n}}\right) \approx 0.13 .
$$

\section{E. Radiative corrections at $T=0$}

\section{Standard computation}

Radiative corrections at $T=0$ correspond to two types of corrections (Abers et al., 1968, Dicus et al. 1982 , Kernan, 1993, Sirlin, 1967). First the radiative corrections per se, that is for which a virtual photon is emitted and 
absorbed inside the interaction and which interfere with the lowest order or Born diagram. Hereafter we call these pure radiative corrections and the relevant diagrams in the infinite nucleon mass limit are depicted in Fig. 13 (see also Ivanov et al. $(2013$, App. C)). As shown in the seminal article by Sirlin (1967), the other diagrams involving virtual photons ${ }^{12}$ can be reabsorbed in the redefinitions of $G_{F}$ and $g_{A}$.

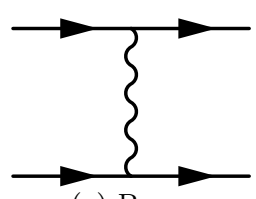

(a) Born

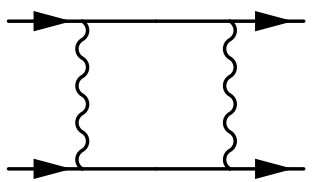

(c)

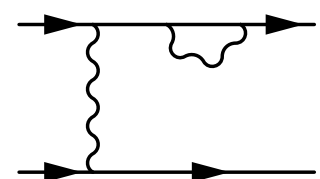

(b)

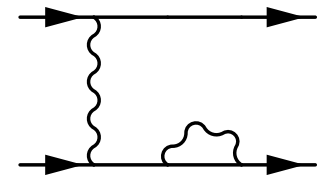

(d)
FIG. 13 Born diagram and virtual photon radiative corrections.

Second it also contains the corrections due to the emission of real photon (Abers et al., 1968, Fig 2e,2f). These processes are generically called bremsstrahlung (BS), and in the infinite nucleon mass approximation only the emission from the electron line (Fig. 14a) contributes.

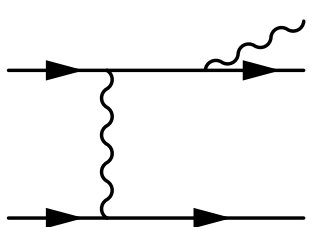

(a)

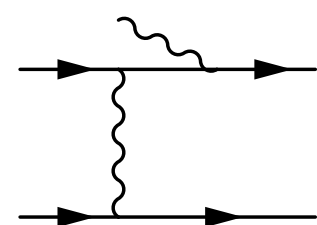

(b)
FIG. 14 Bremsstrahlung (left) and absorption (right).

It is necessary to handle these two types of corrections simultaneously, because each of these contributions contains an infrared divergence in the photon momentum. But as is well known, when both are considered, these divergences exactly cancel provided they are correctly regularized. The usual procedure consists in letting the photon have a mass for the computation of each type of correction, and then noticing that the log-divergences

12 These extra diagrams correspond first to a virtual photon exchange between the electron and the gauge boson, and between the electron and the neutron (Sirlin, 1967 Fig. 1), and second to a virtual photon exchange between the proton and the gauge boson and between the proton and the neutron (Sirlin 1967, Fig. $3)$. which appear are exactly opposite, leading to a finite result in the limit $m_{\gamma} \rightarrow 0$ (Abers et al. 1968 ); (Ivanov et al., 2013, App. B). These radiative corrections are very well understood for the neutron beta decay Abers et al., 1968, Czarnecki et al., 2004, Marciano and Sirlin, 2006: Sirlin, 1967). The main result is that radiative corrections can be taken into account by a multiplicative factor whose expression involves Sirlin's universal function (Sirlin, 1967, Eq. 20b). This was subsequently refined mainly to improve the expression of the high energy cut-off which was related to the mass of the vector boson once weak interactions were properly understood, and we use Czarnecki et al. (2004) as the most accurate account on this issue.

A careful analysis however indicates that only pure radiative corrections take a universal form which can be used for all the reactions 68). They are given by Abers et al. (1968, Eq. 6.2) and depend only on the electron velocity $\beta=p / E^{13}$. The total corrections for the neutron beta decay include also the bremsstrahlung (Abers et al. 1968, Eq. 6.6) and these are computed specifically for the neutron beta decay, with a maximum photon energy being $\Delta-E$.

Hence, the radiative corrections for the reactions (68) should in principle be recomputed to take into account the correct bremsstrahlung corrections for each reaction. However it proves much more convenient to temporarily forget about this issue and assume that the radiative corrections computed for the neutron beta decay can be used without modification for all weak reactions. We postpone the correct treatment of bremsstrahlung to the next section.

Note that even for the neutron beta decay this approximation amounts to ignoring the Pauli-blocking effects of the neutrinos which is modified by the energy subtracted from the photon. In this approximation, the maximum energy of emitted photons is chosen to be the maximum energy of the neutrino when in the final state. For the neutron beta decay this is $\Delta-E$, but for reaction 68c this is $\Delta+E$.

Under this assumption, our method to compute the radiative correction at $T=0$ follows exactly the approach by Dicus et al. (1982) and subsequent literature based on it, for which the same assumption was implicitly made. The radiative corrections can be separated between a Coulomb correction, which accounts for the motion of the electron in the Coulomb field of the proton, and the other corrections. It is customary to factorize the Coulomb corrections as they can be taken into account multiplicatively by the Fermi function ${ }^{14}$. The diagram $13 \mathrm{c}$ corresponds to the interaction between the

13 Even though they have been derived for neutron beta decay, they remain formally identical for other reactions because they are related by crossing symmetry under which $\beta$ is left invariant.

14 This amounts to including some radiative corrections which are higher order in $\alpha_{\mathrm{FS}}$. 
proton and the electron, and since this is accounted for by the Fermi function, its Coulomb part is subtracted so as to avoid double counting.

The non-relativistic Fermi function is

$$
F(E) \equiv \frac{y}{1-\mathrm{e}^{-y}}, \quad y \equiv \frac{2 \pi \alpha_{\mathrm{FS}}}{\beta} \quad \beta=\frac{p}{E} .
$$

The relativistic Fermi function is given by (Ivanov et al. 2013, Eq. 5)

$$
\begin{gathered}
F(E) \equiv \frac{4\left(1+\frac{\gamma}{2}\right)\left(2 r_{p} m_{e} \beta\right)^{2 \gamma}}{\Gamma^{2}(3+2 \gamma)} \frac{\mathrm{e}^{\pi \alpha_{\mathrm{FS}} / \beta}}{\left(1-\beta^{2}\right)^{\gamma}} \\
\times\left|\Gamma\left(1+\gamma+\mathrm{i} \frac{\alpha_{\mathrm{FS}}}{\beta}\right)\right|^{2}
\end{gathered}
$$

where $\gamma \equiv \sqrt{1-\alpha_{\mathrm{FS}}^{2}}-1$ and $r_{p}$ is the proton radius. In practice the non-relativistic function is enough as long as we do not focus on a precision on $Y_{\mathrm{P}}^{(4)}$ better than unity. The modification on light elements yields is evaluated in $\S$ V.C and we find $\Delta Y_{\mathrm{P}}^{(4)} \simeq 0.3$ when using the relativistic Fermi function (see also Smith and Fuller (2010) which reports $\left.\Delta Y_{\mathrm{P}}^{(4)} \simeq 0.4\right)$. In this work we use the relativistic Fermi function whenever it is not specified differently. The relative difference between the two Fermi functions is plotted in Fig. 15 and it remains smaller than $0.06 \%$.

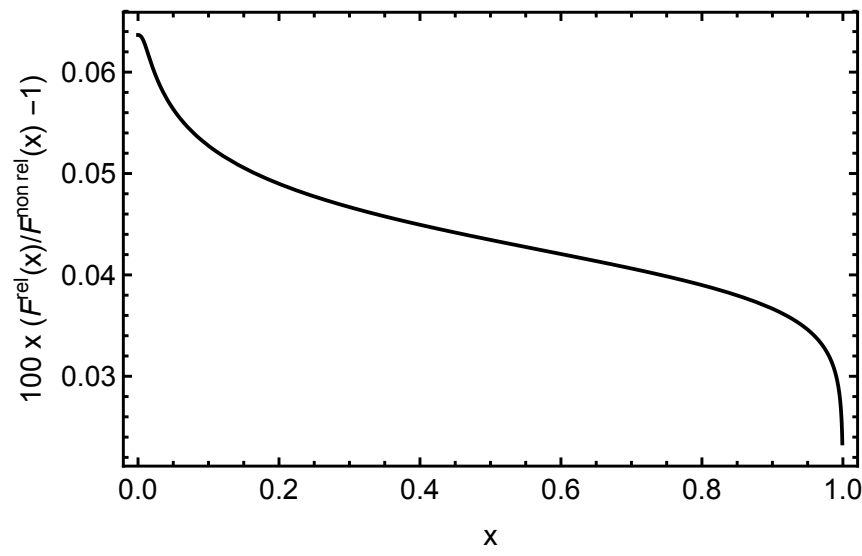

FIG. 15 Relative difference between the relativistic and the non-relativistic Fermi function.

Considering the contributions of Coulomb and other radiative corrections, the Born rates are modified as

$$
\begin{array}{r}
\Gamma_{n \rightarrow p}^{\mathrm{RC} 0}=K \int_{0}^{\infty} p^{2} \mathrm{~d} p\left[\mathcal{F}_{+}(E) R(E,|\Delta-E|) \chi_{+}(E)\right. \\
\left.+\mathcal{F}_{+}(-E) R(E, \Delta+E) \chi_{+}(-E)\right] .(101)
\end{array}
$$

The Coulomb corrections occur only if both the electron and the proton are either in the initial or the final state. We have thus defined the compact notation

$$
\mathcal{F}_{ \pm}(x)=\left\{\begin{array}{lll}
F(|x|) & \text { if } & \pm x>0 \\
1 & \text { if } & \pm x \leq 0
\end{array}\right.
$$

which ensures that this is the case.

The factor $R(.,$.$) takes into account pure radiative cor-$ rections and bremsstrahlung (but only in the form of neutron beta decay in vacuum as evoked above) and is of the form

$$
R\left(E, k_{\max }\right) \equiv 1+\frac{\alpha_{\mathrm{FS}}}{2 \pi} \mathcal{C}\left(E, k_{\max }\right) .
$$

The function $\mathcal{C}$ is given in Eq. B30. However we find it better to use the most recent form (B35) for the radiative correction $R\left(E, k_{\mathrm{kmax}}\right)$ which amounts to resumming higher order corrections, since it is more accurate (Czarnecki et al., 2004; Esposito et al., 1999). We show in $\S$ V.C that it modifies the ${ }^{4} \mathrm{He}$ production by $\Delta Y_{\mathrm{P}}^{(4)} \simeq 0.2$.

The rate for protons is obtained by the replacement $\Delta \rightarrow-\Delta$ in 101 , that is from $\chi_{+} \rightarrow \chi_{-}$, together with the replacement $\mathcal{F}_{+} \rightarrow \mathcal{F}_{-}$to ensure that the Fermi function appears when the proton and the electron are on the same side. Hence it is given by

$$
\begin{aligned}
\Gamma_{p \rightarrow n}^{\mathrm{RC} 0}=K & \int_{0}^{\infty} p^{2} \mathrm{~d} p\left[\mathcal{F}_{-}(E) R(E, E+\Delta) \chi_{-}(E)\right. \\
& \left.+\mathcal{F}_{-}(-E) R(E,|E-\Delta|) \chi_{-}(-E)\right] .(104
\end{aligned}
$$

Given our choice to take into account the bremsstrahlung corrections of neutron beta decay for all processes and our choice for the maximum energy of emitted photons $k_{\max }$, then by construction the detailed balance property 86 is preserved using again the property 85 , that is we get by construction

$$
\Gamma_{p \rightarrow n}^{\mathrm{RC} 0}=\mathrm{e}^{-\Delta / T} \Gamma_{n \rightarrow p}^{\mathrm{RC} 0} .
$$

The relative variations of the weak rates from radiative corrections are plotted in Fig. 16 .

The radiative corrections also affect the free decay rate of neutrons and thus the value of $\lambda_{0}$ which is increased to

$$
\begin{aligned}
\lambda_{0}^{\mathrm{RC} 0} & \equiv m_{e}^{-5} \int_{0}^{\sqrt{\Delta^{2}-m_{e}^{2}}} F(E) R\left(E, E_{\nu}\right) p^{2} E_{\nu}^{2} \mathrm{~d} p \\
& \simeq 1.75767
\end{aligned}
$$

with $E_{\nu}=\Delta-E$.

\section{Bremsstrahlung corrections}

It was argued in Brown and Sawyer (2001) that the detailed balance relation (86) should hold even including radiative corrections at order $\alpha_{\mathrm{FS}}$, as long as we work in the infinite nucleon mass approximation. Hence the derivation of radiative corrections due to interactions with the surrounding bath of particles, which are usually called finite-temperature corrections, must satisfy this detailed balance relation. These corrections correspond to real photon processes which are the absorption diagram $14 \mathrm{~b}$ 


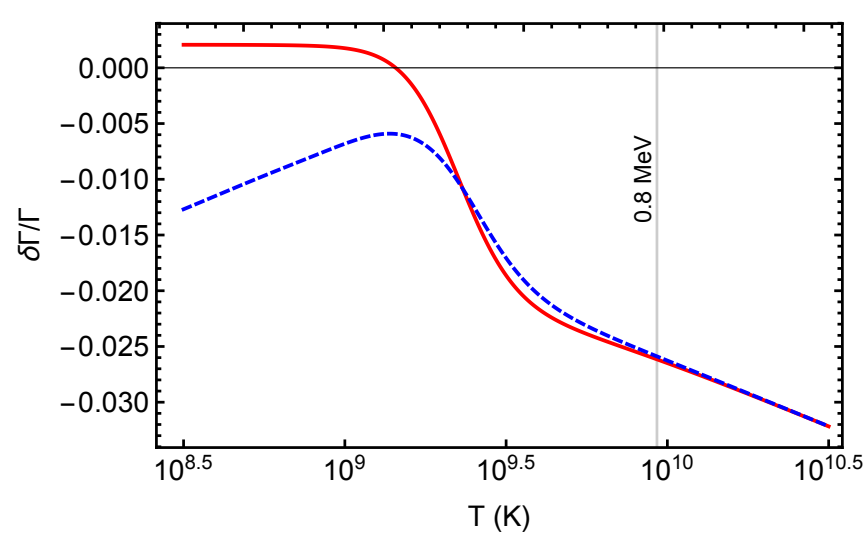

FIG. $16 \delta \Gamma / \Gamma$ due to Coulomb and null-temperature radiative corrections. Red continuous line $: n \rightarrow p$. Blue dashed line: $p \rightarrow n$. The relative variations are equal at high temperature, when $T_{\nu} \simeq T$, and the detailed balance is still satisfied when including these corrections. This plot reproduces Lopez and Turner (1999, Fig. 8).

together with the stimulated emission part of $14 \mathrm{a}$, and to mass shift effects illustrated by the diagrams 17 .

The full computation of the finite temperature radiative corrections was carefully carried out in Brown and Sawyer (2001), following the guideline of detailed balance and with a detailed discussion on misconceptions in earlier efforts (Cambier et al. 1982, Chapman, 1997; Dicus et al., 1982, Esposito et al. 2000a, Sawyer, 1996) concerning the so called wave-function renormalization. However the authors of Brown and Sawyer (2001) reported that when combining their results with the null-temperature radiative corrections discussed in the previous section, the rates failed to satisfy the detailed balance relation. We argue that this is because bremsstrahlung was inconsistently taken into account. As long as we take into account only null-temperature radiative corrections, it is reasonable to consider that the bremsstrahlung effects are those of the neutron beta decay for all reactions, so as to maintain the detailed balance relation (86). This is what has been done in the previous section.

Strictly speaking it is a mistake because part of the null-temperature corrections are left out. However it is the most reasonable procedure if one ignores the full details of the finite-temperature radiative corrections. Here since we take into account the interactions with the bath of photons and electrons in the finite temperature radiative corrections, we must also correct for this ad-hoc and incorrect treatment of bremsstrahlung in the previous section. The details are reported in appendix B.7 and the result is that we need to add the corrections

$$
\begin{array}{r}
\delta \Gamma_{n \rightarrow p}^{\mathrm{BS}}=\frac{\alpha_{\mathrm{FS}} K}{2 \pi} \int_{m_{e}}^{\infty} \mathrm{d} E\left[g(-E) \mathcal{F}_{+}(E) \gamma_{n \rightarrow p+e}^{\mathrm{BS}}\right. \\
\left.+g(E) \mathcal{F}_{+}(-E) \gamma_{n+e \rightarrow p}^{\mathrm{BS}}\right] \\
\delta \Gamma_{p \rightarrow n}^{\mathrm{BS}}=\frac{\alpha_{\mathrm{FS}} K}{2 \pi} \int_{m_{e}}^{\infty} \mathrm{d} E\left[g(-E) \mathcal{F}_{-}(E) \gamma_{p \rightarrow n+e}^{\mathrm{BS}}\right. \\
\left.+g(E) \mathcal{F}_{-}(-E) \gamma_{p+e \rightarrow n}^{\mathrm{BS}}\right],
\end{array}
$$

with the definitions $(\mathrm{B} 48, \mathrm{~B} 49)$. Note that we have added the Fermi factor contribution for consistency with the rest of the radiative corrections. Their temperature dependence is plotted in Fig. 18. Even though these BS corrections correspond to null temperature radiative corrections, unless specified we include them as part of the finite temperature radiative corrections discussed in the next section, since they must be added only to maintain consistently the detailed balance relation when finite temperature radiative corrections are included. We report in $\S \mathrm{V} . \mathrm{C}$ that these BS corrections are responsible for a non-negligible modification of ${ }^{4} \mathrm{He}$ production which is $\Delta Y_{\mathrm{P}}^{(4)} \simeq-3.1$.

\section{F. Finite temperature radiative corrections}

The finite-temperature corrections can be separated in three parts. Using a notation similar to Brown and Sawyer (2001), these three contributions are noted

$$
\Gamma_{n \rightarrow p}^{T} \equiv \Gamma_{n \rightarrow p}^{\gamma, T}+\Gamma_{n \rightarrow p}^{\Delta E, T}+\Gamma_{n \rightarrow p}^{e p+e e, T},
$$

and similar notation for the $p \rightarrow n$ processes.

The first part is the stimulated emission and absorption of real photons (Brown and Sawyer, 2001, Eq. 5.9), see Fig. 14. When combined with a contribution coming from the diagram 17 a it gives

$$
\begin{aligned}
& \Gamma_{n \rightarrow p}^{\gamma, T}=\frac{\alpha_{\mathrm{FS}} K}{2 \pi} \int_{0}^{\infty} \frac{\mathrm{d} k}{k} \int_{m_{e}}^{\infty} \mathrm{d} E \times \\
& \left\{A(E, k)\left[g(-E) \widetilde{\chi}_{+}^{A}(E, k)+g(E) \widetilde{\chi}_{+}^{A}(-E, k)\right]\right. \\
& \left.\quad-k B(E, k)\left[g(-E) \widetilde{\chi}_{+}^{B}(E, k)+g(E) \widetilde{\chi}_{+}^{B}(-E, k)\right]\right\} \\
& \Gamma_{p \rightarrow n}^{\gamma, T}=\left.\Gamma_{n \rightarrow p}^{\gamma, T}\right|_{\widetilde{\chi}_{+}^{A / B} \rightarrow \tilde{\chi}_{-}^{A / B}}
\end{aligned}
$$

with

$$
\begin{aligned}
& \widetilde{\chi}_{ \pm}^{A}(E, k) \equiv \widetilde{\chi}_{ \pm}(E-k)+\widetilde{\chi}_{ \pm}(E+k)-2 \widetilde{\chi}_{ \pm}(E[110) \\
& \widetilde{\chi}_{ \pm}^{B}(E, k) \equiv \pm\left[\widetilde{\chi}_{ \pm}(E-k)-\widetilde{\chi}_{ \pm}(E+k)\right] .
\end{aligned}
$$

It does not satisfy the detailed balance relation. However when the bremsstrahlung corrections (107) are added the sum does satisfy it. This crucial point is detailed in App. B.7.c

The second part of finite temperature radiative corrections is made of the electron energy shift (Brown and 


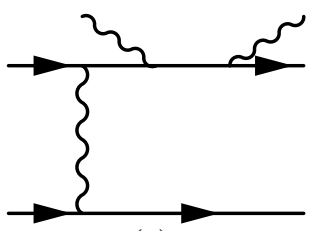

(a)

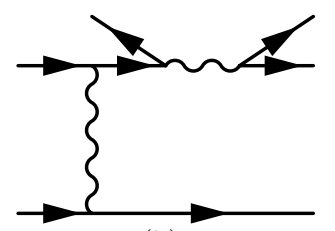

(b)
FIG. 17 Feynman diagram interpretation of finite temperature radiative corrections, that is of the effect of the interactions with the surrounding plasma.

Sawyer, 2001, Eq. 5.13), and it originates partly from the diagrams 17 . It reads

$$
\begin{aligned}
\Gamma_{n \rightarrow p}^{\Delta E, T} & =-\frac{2 \alpha_{\mathrm{FS}} K}{\pi} \int \mathrm{d} p\left[\chi_{+}(E)+\chi_{+}(-E)\right] \quad(112 \mathrm{a}) \\
& \times\left[\frac{\pi^{2} T^{2}}{6}+\int_{m_{e}}^{\infty} \mathrm{d} E^{\prime} p^{\prime} g\left(E^{\prime}\right)\left(1-\frac{m_{e}^{2}}{E^{\prime 2}-E^{2}}\right)\right] \\
\Gamma_{p \rightarrow n}^{\Delta E, T} & =\left.\Gamma_{n \rightarrow p}^{\Delta E, T}\right|_{\chi_{+} \rightarrow \chi_{-}},
\end{aligned}
$$

where $E^{\prime} \equiv \sqrt{{p^{\prime}}^{2}+m_{e}^{2}}$.

The third and last part is made of proton-electron interactions, which is a finite temperature correction to the diagram 13c, and electron self-energy and wave-function renormalization (Brown and Sawyer, 2001, Eq. 5.10), which come partly from the diagram $17 \mathrm{~b}$. It reads

$$
\begin{aligned}
\Gamma_{n \rightarrow p}^{e p+e e, T} & =\frac{\alpha_{\mathrm{FS}} K}{2 \pi} \int_{m_{e}}^{\infty} \mathrm{d} E\left[\chi_{+}(E)+\chi_{+}(-E)\right] \\
& \times \int_{m_{e}}^{\infty} \mathrm{d} E^{\prime} g\left(E^{\prime}\right) \frac{E}{E^{\prime 2}-E^{2}} \\
& \times\left\{8 p p^{\prime}-\left(E^{\prime 2}+E^{2}\right) \ln \left(\frac{p+p^{\prime}}{p-p^{\prime}}\right)^{2}\right. \\
& \left.-\frac{E^{\prime}}{E}\left(E^{\prime 2}+E^{2}\right) \ln \left[\frac{\left(E E^{\prime}+p p^{\prime}\right)^{2}-m_{e}^{4}}{\left(E E^{\prime}-p p^{\prime}\right)^{2}-m_{e}^{4}}\right]\right\} \\
\Gamma_{p \rightarrow n}^{e p+e e, T} & =\left.\Gamma_{n \rightarrow p}^{e p+e e, T}\right|_{\chi_{+} \rightarrow \chi_{-}} \cdot
\end{aligned}
$$

The second and third contributions [Eqs. 112 and (113)] satisfy manifestly the detailed balance relation thanks to the property (85). Furthermore, they can be recast in a form which does not involve principal parts of integrals and are thus better suited for numerical integration (Brown and Sawyer, 2001, Eq 5.15)]. We report it in App. B.8.

The modification of the rates from these finite temperature corrections is plotted in Fig. 18. We also show the BS corrections and the sum of the two. By construction for the sum, it can be checked that whenever the neutrinos have the same temperature, that is for $T>10^{10} \mathrm{~K}$, the relative variation of the rates are equal and the detailed balance property 86 is preserved.

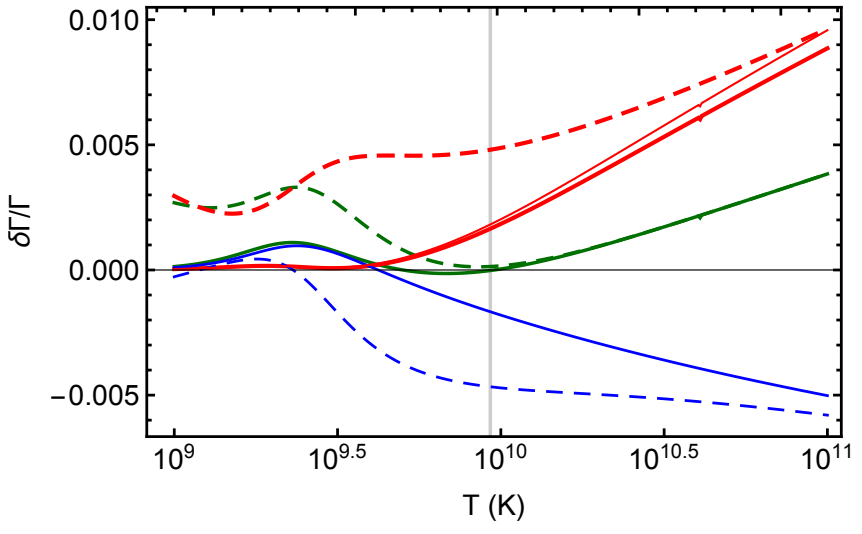

FIG. $18 \delta \Gamma / \Gamma$ from finite-temperature radiative corrections. Continuous line $: n \rightarrow p$. Dashed line $: p \rightarrow n$. The red lines are the corrections of Brown and Sawyer (2001) in very good agreement with their Fig. 2. In the thin continuous red line, the $n+\bar{\nu}+e^{+} \rightarrow p+\gamma$ has been added to the $n \rightarrow p$ corrections, as advocated in Brown and Sawyer (2001) and we check that it results in a very small modification, as also found in their Fig. 3, and most notably it is insufficient to satisfy detailed balance. The blue lines are the Bremsstrahlung corrections (107). Finally the green lines are the total corrections, that is the corrections of (Brown and Sawyer, 2001) with all bremsstrahlung corrections added. We check that above $T>10^{10} \mathrm{~K}$, that is when $T_{\nu}=T$, the relative variations of the total corrections are equal, implying that the detailed balance is satisfied.

\section{G. Finite nucleon mass corrections}

It is not fully correct to consider that nucleons have an infinite mass. Indeed, the typical energy transfer in weak-interactions to electrons and neutrinos is of the order of the mass gap $\Delta \simeq 1.29 \mathrm{MeV}$, which is $1.4 \times 10^{-3}$ smaller than the nucleon mass. It corresponds to a temperature of $1.5 \times 10^{10} \mathrm{~K}$ which is not much larger than the freeze-out temperature. In the infinite nucleon mass approximation, we have thus neglected factors of the type $E_{\nu} / m_{N}, E_{e} / m_{N}$ or $\Delta / m_{N}$ (where $m_{N}$ is the average nucleon mass $\left.m_{N} \equiv\left(m_{p}+m_{n}\right) / 2\right)$ which represent order $10^{-3}$ corrections with respect to the leading one around $10^{10} \mathrm{~K}$ and even larger corrections at higher temperature. Our method consists in expanding the full reaction rate in power of a small parameter $\epsilon \equiv T / m_{N}$. Terms of the type $E_{\nu} / m_{N}$ or $E_{e} / m_{N}$ are obviously of order $\epsilon$ and terms of the type $\Delta / m_{N}$ are also treated as being of or$\operatorname{der} \epsilon$. Our implementation of the finite mass corrections consists in including all the terms up to order $\epsilon$, but neglecting terms of order $\epsilon^{2}$. This means that we neglect terms whose importance is of order $10^{-6}$ which is justified given our goal in precision.

If we ignore radiative corrections at null temperature, 
these corrections take the form

$$
\begin{aligned}
\delta \Gamma_{n \rightarrow p}^{\mathrm{FM}} & =K \int_{0}^{\infty} p^{2} \mathrm{~d} p \\
& \times\left[\chi_{+}^{\mathrm{FM}}\left(E, g_{A}\right)+\chi_{+}^{\mathrm{FM}}\left(-E, g_{A}\right)\right] \\
\delta \Gamma_{p \rightarrow n}^{\mathrm{FM}} & =K \int_{0}^{\infty} p^{2} \mathrm{~d} p \\
& \times\left[\chi_{-}^{\mathrm{FM}}\left(E,-g_{A}\right)+\chi_{-}^{\mathrm{FM}}\left(-E,-g_{A}\right)\right],
\end{aligned}
$$

and the functions $\chi_{ \pm}^{\mathrm{FM}}$ are reported in App. B.3. However since finite mass effects and radiative corrections can be rather important, they cannot be added linearly and one should also include radiative corrections inside finite nucleon mass corrections ${ }^{15}$. Hence the corrections to the rates are

$$
\begin{aligned}
\delta \Gamma_{n \rightarrow p}^{\mathrm{RC}+\mathrm{FM}}= & K \int_{0}^{\infty} p^{2} \mathrm{~d} p \\
\times & {\left[\mathcal{F}_{+}(E) R(E,|E-\Delta|) \chi_{+}^{\mathrm{FM}}\left(E, g_{A}\right)\right.} \\
& \left.+\mathcal{F}_{+}(-E) R(E, E+\Delta) \chi_{+}^{\mathrm{FM}}\left(-E, g_{A}\right)\right] \\
\delta \Gamma_{p \rightarrow n}^{\mathrm{RC}+\mathrm{FM}}= & K \int_{0}^{\infty} p^{2} \mathrm{~d} p \\
\times & {\left[\mathcal{F}_{-}(E) R(E, E+\Delta) \chi_{-}^{\mathrm{FM}}\left(E,-g_{A}\right)\right.} \\
& \left.+\mathcal{F}_{-}(-E) R(E,|E-\Delta|) \chi_{-}^{\mathrm{FM}}\left(-E,-g_{A}\right)\right] .
\end{aligned}
$$

The expression for $\chi_{ \pm}^{\mathrm{FM}}\left(E, g_{A}\right)$ is given in App. B.3. The relative modifications to the rates are depicted in Fig. 19 It is not obvious that the finite nucleon mass corrections preserve the detailed balance relation. In fact when including these corrections the detailed balance ratio between neutrons and protons is given by Eq. A15. Since this must also be the ratio $\Gamma_{p \rightarrow n} / \Gamma_{n \rightarrow p}$ we define $\alpha$ following Lopez et al. (1997) as

$$
\frac{\bar{\Gamma}_{p \rightarrow n}+\delta \Gamma_{p \rightarrow n}^{\mathrm{FM}}}{\bar{\Gamma}_{n \rightarrow p}+\delta \Gamma_{n \rightarrow p}^{\mathrm{FM}}} \equiv \mathrm{e}^{-\frac{\Delta}{T}}\left[1+(1+\alpha) \frac{3 \Delta}{2 m_{N}}\right] .
$$

$\alpha$ characterizes the deviation from the detailed balance equality and must vanish if detailed balance with finite mass corrections is satisfied. It is plotted in Fig. 20. We observe that for $T<m_{e}$ deviations from detailed balance occur because the neutrino temperature is not equal to the plasma temperature. Hence we have also plotted $\alpha$ with $T_{\nu}=T$ artificially enforced. At low temperature, that is below $T=m_{e}$, detailed balance is very well satisfied because the parameter $\epsilon=T / m_{N}$ is small. At higher temperatures we see deviations, and this comes from the

${ }^{15}$ In principle one should rederive all radiative corrections including consistently all finite nucleon mass effects. This is absent from the literature, and is certainly a daunting task. Our procedure consists in postulating that the multiplicative factor introduced by radiative corrections also multiplies the finite nucleon mass correction as in Cooper et al. (2010); Czarnecki et al. (2004)

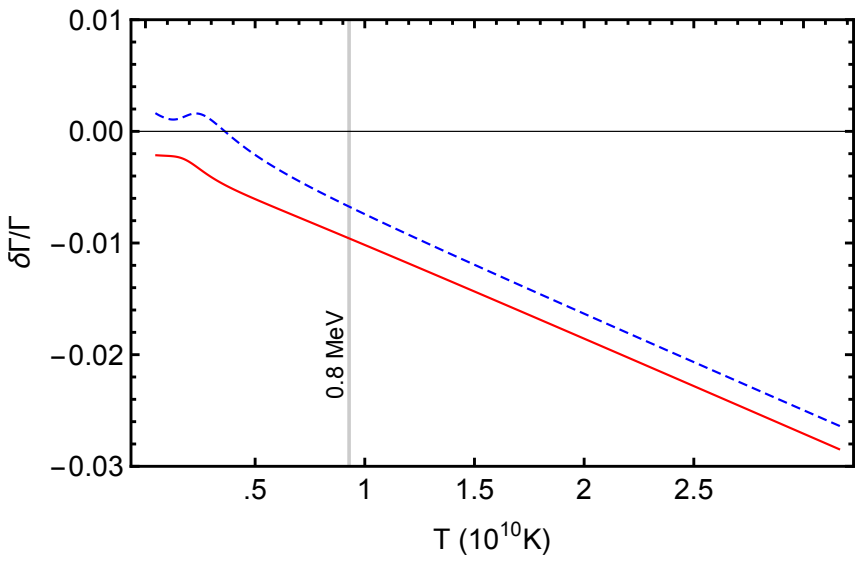

FIG. $19|\delta \Gamma / \bar{\Gamma}|$ from finite nucleon mass effects. Red continuous line $: n \rightarrow p$. Blue dashed line $: p \rightarrow n$. This plot is to be compared with Lopez and Turner (1999, Fig. 9).

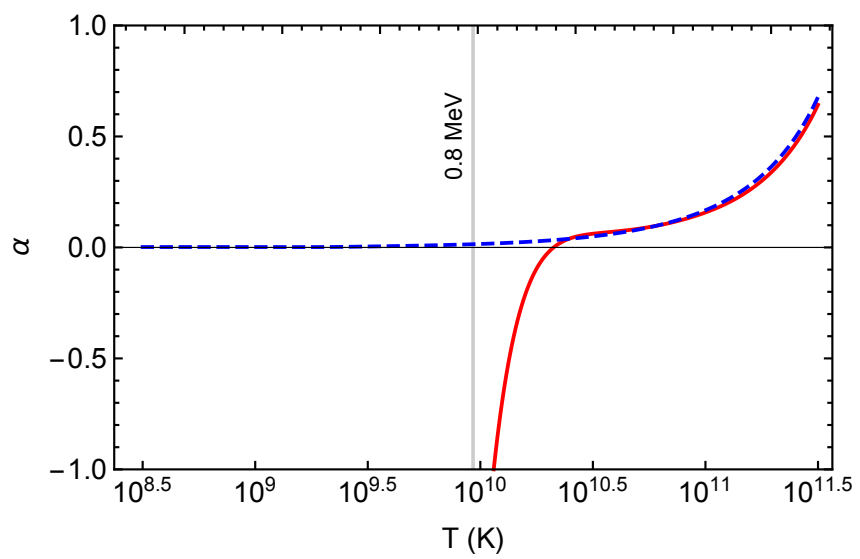

FIG. $20 \alpha$ defined in Eq. 116. Red continuous line: With $T_{\nu}$ differing from $T$ when electron-positron annihilations occur. Blue dashed line: With $T_{\nu}=T$ artificially enforced. This plot is to be compared with Lopez et al. (1997, Fig. 4).

fact that we considered only corrections which are first order in $\epsilon$.

Finally, the total neutron rate at null temperature is

$$
\Gamma_{n \rightarrow p}(T=0)=\Gamma_{n \rightarrow p}^{\mathrm{RC} 0}(T=0)+\delta \Gamma_{n \rightarrow p}^{\mathrm{FM}}(T=0),
$$

that is there is a correction to $\lambda_{0}$ due to finite nucleon mass effects. If Eq. (114) is used, this is

$$
\delta \lambda_{0}^{\mathrm{FM}}=\left.m_{e}^{-5} \int_{0}^{\sqrt{\Delta^{2}-m_{e}^{2}}} p^{2} \mathrm{~d} p \chi_{+}^{\mathrm{FM}}\left(E, g_{A}\right)\right|_{T=0}
$$

combined with Eq. B26, and we obtain $\delta \lambda_{0}^{\mathrm{FM}} \simeq$ $-3.3828 \times 10^{-3}$, that is $\delta \lambda_{0}^{\mathrm{F} M} / \bar{\lambda}_{0} \simeq-0.002068$. This is in agreement with the result $\delta \lambda_{0}^{\mathrm{FM}} / \bar{\lambda}_{0} \simeq-0.00206$ found in Lopez et al. (1997) with an exact method to compute the finite nucleon mass effects. We recomputed this ratio with the method of Lopez et al. (1997, Eq. 20) and found it is more precisely given by -0.0020637 . The tiny difference with our value $\delta \lambda_{0}^{\mathrm{FM}} / \bar{\lambda}_{0}$ is only due to the fact 
that we kept only first order finite nucleon mass corrections, that is we kept terms of order $\epsilon$, and ignored terms of higher order $\epsilon^{2}$. Furthermore, if we also include radiative corrections, that is if we use $\delta \Gamma_{n \rightarrow p}^{\mathrm{RC}+\mathrm{FM}}$ instead of $\delta \Gamma_{n \rightarrow p}^{\mathrm{FM}}$ in Eq. 117), hence corresponding to an extra factor $F(E) R(E, \Delta-E)$ in the integrand of Eq. (118), we get $\delta \lambda_{0}^{\mathrm{FM}} \simeq-3.6201 \times 10^{-3}$. These values are summarized in table $\amalg$.

\section{H. Weak magnetism}

We show in B.4 that the effect of weak magnetism is exactly similar to the finite mass correction which arises from the coupling between the axial current and the vector current. This has been noticed earlier by Seckel (1993). That is, it brings corrections which are exactly of the same type as those proportional to $g_{A}$ in Eq. 733 . The weak magnetism corrections amount to a simple redefinition of the constant factors (B11) as

$$
\begin{aligned}
c_{L L} & \equiv \frac{\left(1+g_{A}\right)^{2}}{4}+f_{\mathrm{wm}} g_{A} \\
c_{R R} & \equiv \frac{\left(1-g_{A}\right)^{2}}{4}-f_{\mathrm{wm}} g_{A} \\
c_{L R} & \equiv \frac{g_{A}^{2}-1}{4}
\end{aligned}
$$

when computing the finite nucleon mass corrections. The associated couplings (B24) must be replaced accordingly.

The weak magnetism, if considered independently of radiative corrections, induces no modification of $\lambda_{0}$, at least up to the order $\epsilon$ of our finite nucleon mass expansion, as explained in appendix $\S$ B.4. However when this is combined with the radiative corrections, this brings a residual increase to $\delta \lambda_{0}^{\mathrm{FM}} \simeq-3.6333 \times 10^{-3}$. Summing this value of the finite nucleon mass corrections (which includes weak-magnetism and which is coupled to radiative corrections), to the radiative corrections themselves (106) leads to

$$
\lambda_{0}^{\mathrm{RC}+\mathrm{FM}}=\lambda_{0}^{\mathrm{RC}}+\delta \lambda_{0}^{\mathrm{FM}} \simeq 1.75474 .
$$

This is to be compared with Cooper et al. (2010) where it is reported $\lambda_{0} \simeq 1.03887 \times 1.6887 \simeq 1.75434$, a modest $0.023 \%$ smaller than the value 120 . This close agreement can also be seen by noting that $\left(\hbar 2 \pi^{3}\right) /\left(m_{e}^{5} c^{10} G_{F}^{2} \lambda_{0}^{\mathrm{RC}+\mathrm{FM}}\right) \simeq 4907.4 \mathrm{~s}$, which is very close to the value $4908 \mathrm{~s}$ given by Czarnecki et al. (2004 Eq. 17) or the refined value $4908.7 \mathrm{~s}$ of Marciano and Sirlin (2006, Eq. 18).

\section{Effect of incomplete neutrino decoupling}

Neutrino heating also induces modification of the weak rates. In our thermal approximation of the incomplete neutrino decoupling (see $\S$ II.F), this is taken into account by putting the effective neutrino temperature defined in Eq. (64) in all expressions for the weak rates.
TABLE II Value of $\delta \lambda_{0}^{\mathrm{FM}}$ depending on the effects considered.

\begin{tabular}{cccc}
\hline RC & FM & WM & FM+WM \\
\hline No & $-3.3828 \times 10^{-3}$ & 0 & $-3.3828 \times 10^{-3}$ \\
Yes & $-3.6201 \times 10^{-3}$ & $-0.0132 \times 10^{-3}$ & $-3.6333 \times 10^{-3}$ \\
\hline
\end{tabular}

This amounts to assuming that all neutrinos receive the same share of the heating and ignoring the spectral distortions. We postpone a more detailed discussion on the effect of incomplete neutrino decoupling on the weak rates and thus the ${ }^{4} \mathrm{He}$ production in $\S$ V.C.5. Briefly, the most notable effect of incomplete neutrino decoupling is to affect the time-temperature relation, and this is also the case from QED corrections in the plasma but the effect is much smaller. Indeed, since for a given plasma temperature, neutrino heating induces an increase of the total energy density, the Hubble rate is increased and so is the rate of variation $\mathrm{d} T / \mathrm{d} t$. In practice this means that the Universe is younger when nucleosynthesis starts around $T_{\mathrm{Nuc}}=0.078 \mathrm{MeV}$ and the neutron beta decay results in a lower loss of neutrons, and thus a higher production of ${ }^{4} \mathrm{He}$ at the end of the BBN. This is the clock effect explained in Dodelson and Turner (1992); Fields et al. (1993).

\section{J. Total correction to the weak rates}

The total weak reaction rates are given by summing the various effects [Eqs. 101), 108, (107) and (115)], that is

$$
\Gamma_{n \rightarrow p}=\Gamma_{n \rightarrow p}^{\mathrm{RC} 0}+\Gamma_{n \rightarrow p}^{T}+\delta \Gamma_{n \rightarrow p}^{\mathrm{BS}}+\delta \Gamma_{n \rightarrow p}^{\mathrm{FM}},
$$

and a similar sum for $p \rightarrow n$ processes. The constant $K$ involved in all contributions is then calibrated on neutron beta decay from $\lambda_{0}$ given by Eq. 120 replaced in Eq. (91). We recall that when including weak-magnetism in finite nucleon mass effects, that is in Eqs. 1115), one must use the constants $(119)$ in (B24), so as to evaluate the $\chi_{ \pm}^{\mathrm{FM}}$ given by Eqs. $\mathrm{B} 23$.

The size of all corrections relative to the Born approximation is plotted in Fig. 21. Around $T \simeq 3.3 \times 10^{9} \mathrm{~K}$ for which the neutron are only subject to beta decay, we find $\delta \Gamma_{n \rightarrow p} / \bar{\Gamma}_{n \rightarrow p} \simeq-0.023$. From the rule 7 this should lead to $\Delta Y_{\mathrm{P}}^{(4)} \simeq 42$, quite in nice agreement with the total correction $\Delta Y_{\mathrm{P}}^{(4)} \simeq 44.7$ that we report in $\S \mathrm{V}$.C

\section{NUCLEOSYNTHESIS}

Once the weak interaction rates are determined and computed with great precision, it is possible to solve for the nucleosynthesis by adding the effect of nuclear reactions which form nuclei. Hence we need to build the 


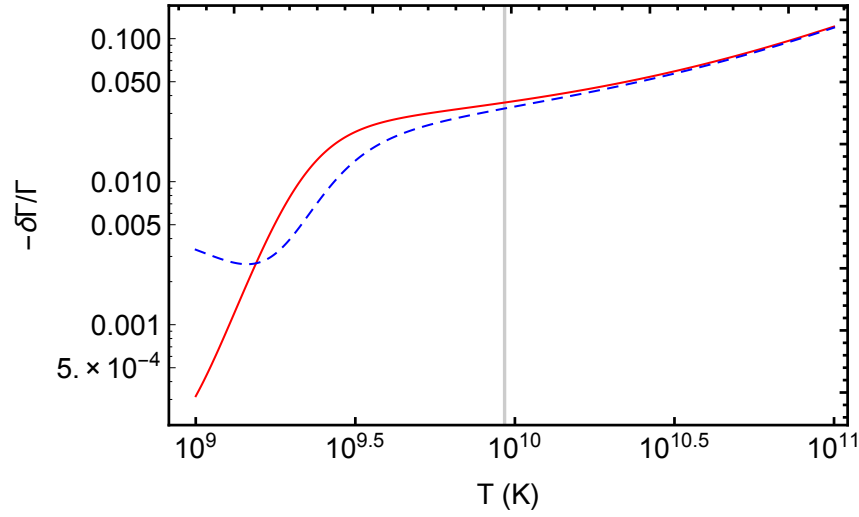

FIG. 21 Total relative rate corrections, including all the effects discussed, $-\delta \Gamma / \bar{\Gamma} . n \rightarrow p$ in red continuous line and $p \rightarrow n$ in blue dashed line. The vertical line corresponds to $T_{F}=0.8 \mathrm{MeV}$.

differential system which rules the evolution of all (neutrons, protons and main isotopes) abundances. We describe how this is performed in this section and turn to report and discuss the numerical results obtained for final abundances in $\S \mathrm{V}$.

We remind that, for a general reaction of the type

$$
A+b \rightarrow C+d
$$

the conventional notation in experimental nuclear physics is $A(b, d) C$ to keep in mind that $A$ is the target nucleus at rest, $b$ is the projectile from the beam, $d$ is the outgoing, detected, particle and $C$ is left generally undetected, possibly not escaping the target. Hence, usually, but not always, $A$ and $C$ are the heaviest nuclei. The same notation is used in theoretical nuclear physics, regardless of the experimental details.

\section{A. Thermonuclear reaction rates}

We summarize here a few results, to be used in this review, and refer to (Angulo et al., 1999, Clayton, 1983, Iliadis, 2007; Longland et al. 2010) for a detailed treatment. It is assumed that the medium is in local thermodynamical equilibrium so that the distribution of ion velocities/energies follows a Maxwell-Boltzmann distribution (see $\S$ A.3),

$$
\phi_{\mathrm{MB}}(v) v \mathrm{~d} v=\sqrt{\frac{8}{\pi m}} \frac{1}{\left(k_{B} T\right)^{3 / 2}} e^{-\frac{E}{k_{B} T}} E \mathrm{~d} E .
$$

It is understood that for the distribution of relative velocities between two reacting nuclei, $m$ is their reduced mass. In such conditions, one defines the thermonuclear reaction rate by

$$
N_{A}\langle\sigma v\rangle=N_{A} \int_{0}^{\infty} \sigma(v) \phi_{\mathrm{MB}}(v) v d v
$$

in $\mathrm{cm}^{3} \mathrm{~s}^{-1} \mathrm{~mole}^{-1}$ units where $N_{A}$ is Avogadro's number $\left(\right.$ mole $\left.^{-1}\right)$. For reactions involving charged particles, since the kinetic energies are below the Coulomb barrier, the energy dependence of the cross section is dominated by the tuneling effect through the barrier. The Coulomb plus centrifugal barrier penetration probability is given by

$$
P_{\ell}(E)=\frac{k R}{F_{\ell}^{2}(\eta, k R)+G_{\ell}^{2}(\eta, k R)}
$$

where $F_{\ell}$ and $G_{\ell}$ are the Coulomb functions (Fröberg, 1955), $k=\sqrt{2 m E} / \hbar$ is the wave number, $\ell$ the orbital angular momentum and

$$
\eta \equiv \frac{Z_{1} Z_{2} e^{2}}{\hbar v}
$$

the Sommerfeld parameter. To account for this strong energy dependency of the cross section, it is convenient to introduce the astrophysical $S$-factor:

$$
\sigma(E) \equiv \frac{S(E)}{E} \exp (-2 \pi \eta)
$$

which, in the absence of resonances removes most of the energy dependence. A resonance, associated to a nuclear level in the compound nucleus formed by the fusion of the projectile and target nuclei, induces a strong but localized variation of the $S$-factor. Hence, the presence of a resonance can increase by several orders of magnitude a reaction rate.

\section{B. General form}

The evolution of abundances, defined in Eq. (1), is deduced from the evolution of number densities found from Eq. (10). Since both nuclear and weak reactions preserve the number of baryons, we obtain

$$
\frac{\mathrm{d} n_{\mathrm{b}}}{\mathrm{d} t}+3 H n_{\mathrm{b}}=0,
$$

that is baryon volume density is only affected by dilution and $n_{\mathrm{b}} \propto 1 / a^{3}$.

For a given isotope $i$, the evolution of the volume density depends on the reaction rates in which it is involved. It is of the form

$$
\frac{\mathrm{d} n_{i}}{\mathrm{~d} t}+3 H n_{i}=\mathcal{J}_{i},
$$

where $\mathcal{J}_{i}$ is the net rate of evolution of number density due to all nuclear reactions. A decay of species $i(i \rightarrow \ldots)$ and a decay reaction in which species $i$ is the end product $(j \rightarrow i+\ldots)$ contribute as

$$
\mathcal{J}_{i} \supset-n_{i} \Gamma_{i} \rightarrow \ldots+n_{j} \Gamma_{j \rightarrow i+\ldots}
$$

where the $\Gamma$ are the decay rates (usually given in $\mathrm{s}^{-1}$ ). A two-body reaction of the type $i+j \leftrightarrow k+l$ contributes instead as

$$
\mathcal{J}_{i} \supset n_{k} n_{l} \gamma_{k l \rightarrow i j}-n_{i} n_{j} \gamma_{i j \rightarrow k l} .
$$


with

$$
\gamma_{i j \rightarrow k l} \equiv\langle\sigma v\rangle_{i j \rightarrow k l} .
$$

Since both the individual $n_{i}$ and the total $n_{\mathrm{b}}$ are affected similarly by expansion, it proves much simpler to study directly the evolution of abundances defined as ${ }^{16}$

$$
Y_{i} \equiv \frac{n_{i}}{n_{\mathrm{b}}}
$$

since one finds

$$
\dot{Y}_{i}=C\left[Y_{i}\right], \quad C\left[Y_{i}\right] \equiv \frac{\mathcal{J}_{i}}{n_{\mathrm{b}}} .
$$

Obviously the decay reactions considered in Eq. 130 contribute as

$$
C\left[Y_{i}\right] \supset-Y_{i} \Gamma_{i} \rightarrow \ldots+Y_{j} \Gamma_{j \rightarrow i+\ldots} .
$$

However two-body reactions contribute as

$$
C\left[Y_{i}\right] \supset Y_{k} Y_{l} \Gamma_{k l \rightarrow i j}-Y_{i} Y_{j} \Gamma_{i j \rightarrow k l},
$$

where we related the rates for abundances to those of number densities through

$$
\Gamma_{i j \rightarrow k l} \equiv n_{\mathrm{b}} \gamma_{i j \rightarrow k l}
$$

Eq. (131) is straightforwardly generalized to reactions with more bodies. In full generality, and without restricting to decay reactions or two-body reactions, the evolution of abundances takes the form (Fowler et al. 1967; Wagoner, 1969)

$$
\dot{Y}_{i_{1}}=\sum_{i_{2} \ldots i_{p}, j_{1} \ldots j_{q}} N_{i_{1}}\left(\Gamma_{j_{1} \ldots j_{q} \rightarrow i_{1} \ldots i_{p}} \frac{Y_{j_{1}}^{N_{j_{1}}} \ldots Y_{j_{q}}^{N_{j_{q}}}}{N_{j_{1}} ! \ldots N_{j_{q}} !}-\Gamma_{i_{1} \ldots i_{p} \rightarrow j_{1} \ldots j_{q}} \frac{Y_{i_{1}}^{N_{i_{1}}} \ldots Y_{i_{p}}^{N_{i_{p}}}}{N_{i_{1}} ! \ldots N_{i_{p}} !}\right)
$$

where $N_{i}$ is the stoichiometric coefficient of species $i$ in the reaction and with the relation between abundance rates and number density rates given by

$$
\Gamma_{i_{1} \ldots i_{p} \rightarrow j_{1} \ldots j_{q}}=n_{\mathrm{b}}^{\left(N_{i_{1}}+\cdots+N_{i_{p}}\right)-1} \gamma_{i_{1} \ldots i_{p} \rightarrow j_{1} \ldots j_{q}} .
$$

Note that for a decay reaction $\Gamma_{i \rightarrow \ldots}=\gamma_{i \rightarrow \ldots}$.

In practice the reaction rates for many-body reactions are given as tables for the quantities $N_{A}^{\left(N_{i_{1}}+\cdots+N_{i_{p}}\right)-1} \gamma_{i_{1} \ldots i_{p} \rightarrow j_{1} \ldots j_{q}}$, as detailed in App. C on the steps required to deduce them from the nuclear physics tables. Furthermore the rates are only given for forward reactions. Indeed, since when there is nuclear statistical equilibrium (NSE) reverse reactions should balance with forward reactions, we can always relate the reverse reactions to the forward reactions from the detailed balance relation

$$
\frac{\gamma_{i_{1} \ldots i_{p} \rightarrow j_{1} \ldots j_{q}}}{\gamma_{j_{1} \ldots j_{q} \rightarrow i_{1} \ldots i_{p}}}=\frac{N_{i_{1}} ! \ldots N_{i_{p}} !}{N_{j_{1}} ! \ldots N_{j_{q}} !}\left[\frac{n_{j_{1}}^{N_{j_{1}}} \ldots n_{j_{q}}^{N_{j_{q}}}}{n_{i_{1}}^{N_{i_{1}}} \ldots n_{i_{p}}^{N_{i_{p}}}}\right]^{\mathrm{NSE}}
$$

where the NSE densities are given in Eq. A22. Since nuclear reactions in many-body reactions do not change the nature of nucleons ${ }^{17}$, that is they conserve the number of protons and neutrons, then using the relation 139

17 This is not the case for decay reactions but for these we neglect the reverse reactions. the reverse reactions are related to the forward reactions by

$$
\begin{aligned}
\frac{\gamma_{j_{1} \ldots j_{q} \rightarrow i_{1} \ldots i_{p}}}{\gamma_{i_{1} \ldots i_{p} \rightarrow j_{1} \ldots j_{q}}} & =\frac{\Pi_{i=i_{1} \ldots i_{p}} \frac{1}{N_{i} !}\left[g_{i}\left(\frac{m_{i} T}{2 \pi}\right)^{3 / 2}\right]^{N_{i}}}{\Pi_{j=j_{1} \ldots j_{q} \frac{1}{N_{j} !}\left[g_{j}\left(\frac{m_{j} T}{2 \pi}\right)^{3 / 2}\right]^{N_{j}}}} \\
& \times \exp \left(\frac{\sum_{j=1}^{q} m_{j}-\sum_{i=1}^{p} m_{i}}{T}\right) .(1
\end{aligned}
$$

We check easily that we recover the particular cases of Fowler et al. (1967, Eqs. 15, 18, 25). Hence the relation between forward and reverse reactions is of the form

$$
\frac{\gamma_{j_{1} \ldots j_{q} \rightarrow i_{1} \ldots i_{p}}}{\gamma_{i_{1} \ldots i_{p} \rightarrow j_{1} \ldots j_{q}}}=\alpha\left(\frac{T}{10^{9} \mathrm{~K}}\right)^{\beta} \exp \left(\frac{\gamma \times 10^{9} \mathrm{~K}}{T}\right),
$$

which depends only on 3 constants. In practice, for a given reaction, the forward reaction rate is tabulated for various values of $T$ or is approximated by an analytic fit, and the constants $(\alpha, \beta, \gamma)$ needed to obtain the reverse reaction rate are also computed once for all from Eq. 141 using the tabulated masses and spins of isotopes [we use the table nubase2016. asc described in Audi et al. (2017)].

\section{Nuclear network and reaction rates uncertainties}

The nuclear reaction network used here has been fully described in Coc et al. (2012): it includes 59 nuclides 
from neutron to ${ }^{23} \mathrm{Na}$ (see table III), linked by 391 reactions involving neutrons, protons, deuterium, tritium $\left({ }^{3} \mathrm{H}\right),{ }^{3} \mathrm{He}$ and $\alpha$-particles induced reactions, and $33 \beta$ decay processes. The complete list of reactions can be found in Table 4 of Coc et al. (2012), together with the references for the values of the reaction rates. Each of these reactions is systematically supplemented $(i j \rightarrow k l)$ by its reverse $(k l \rightarrow i j)$ whose rate is obtained as described above, except for decay reactions whose rates are from Audi et al. (2017).

This network is adapted to the prediction of the primordial abundances of the light elements, but also to the calculation of the abundances of the ${ }^{6} \mathrm{Li},{ }^{9} \mathrm{Be},{ }^{10} \mathrm{~B},{ }^{11} \mathrm{~B}$ and CNO isotopes. As listed in Table 4 of Coc et al. (2012), reaction rates and their associated uncertainties were taken primarily from Angulo et al. (1999); Descouvemont et al. (2004); Iliadis et al. (2010); Xu et al. (2013) evaluations when available. For many reactions, in the absence of sufficient experimental data, the rates come from theory (TALYS code) (Goriely et al., 2008). An extensive sensitivity study, performed by Coc et al. (2012), identified ten reactions that needed further analyses, and it was followed by their re-evaluations (shown in boldface in Table 4 of Coc et al. (2012)). Finally, out of these hundreds of reactions, the most important ones are displayed in Fig. 22 They were identified in sensitivity studies, i.e. Coc and Vangioni, 2010, Cyburt, 2004 Nollett and Burles, 2000) for the light elements $\left({ }^{4} \mathrm{He}, \mathrm{D}\right.$, ${ }^{3} \mathrm{He}$ and ${ }^{7} \mathrm{Li}$ ) and Coc et al. (2012); Coc et al. (2014) for others and correspond to the main nuclear flow.

TABLE III Nuclides considered in the nuclear network.

\begin{tabular}{|c|c|c|c|c|c|c|c|c|c|c|c|c|c|c|}
\hline$Z^{N}$ & 0 & 1 & 2 & 3 & 4 & 5 & 6 & 7 & 8 & 9 & 10 & 11 & 12 & 13 \\
\hline 0 & & $\mathrm{n}$ & & & & & & & & & & & & \\
\hline 1 & $\mathrm{H}$ & ${ }^{2} \mathrm{H}$ & ${ }^{3} \mathrm{H}$ & & & & & & & & & & & \\
\hline 2 & & ${ }^{3} \mathrm{He}$ & ${ }^{4} \mathrm{He}$ & $5 \mathrm{He}$ & ${ }^{6} \mathrm{He}$ & & & & & & & & & \\
\hline 3 & & & & ${ }^{6} \mathrm{Li}$ & ${ }^{7} \mathrm{Li}$ & ${ }^{8} \mathrm{Li}$ & ${ }^{9} \mathrm{Li}$ & & & & & & & \\
\hline 4 & & & & ${ }^{7} \mathrm{Be}$ & ${ }^{8} \mathrm{Be}$ & ${ }^{9} \mathrm{Be}$ & ${ }^{10} \mathrm{Be}$ & ${ }^{11} \mathrm{Be}$ & ${ }^{12} \mathrm{Be}$ & & & & & \\
\hline 5 & & & & ${ }^{8} \mathrm{~B}$ & $\begin{array}{l}{ }^{9} \mathrm{~B} \\
\end{array}$ & ${ }^{10} \mathrm{~B}$ & ${ }^{11} \mathrm{~B}$ & ${ }^{12} \mathrm{~B}$ & ${ }^{13} \mathrm{~B}$ & ${ }^{14} \mathrm{~B}$ & ${ }^{15} \mathrm{~B}$ & & & \\
\hline 6 & & & & ${ }^{9} \mathrm{C}$ & ${ }^{10} \mathrm{C}$ & ${ }^{11} \mathrm{C}$ & ${ }^{12} \mathrm{C}$ & ${ }^{13} \mathrm{C}$ & ${ }^{14} \mathrm{C}$ & ${ }^{15} \mathrm{C}$ & ${ }^{16} \mathrm{C}$ & & & \\
\hline 7 & & & & & & ${ }^{12} \mathrm{~N}$ & ${ }^{13} \mathrm{~N}$ & ${ }^{14} \mathrm{~N}$ & ${ }^{15} \mathrm{~N}$ & ${ }^{16} \mathrm{~N}$ & ${ }^{17} \mathrm{~N}$ & & & \\
\hline 8 & & & & & & ${ }^{13} \mathrm{O}$ & ${ }^{14} \mathrm{O}$ & ${ }^{15} \mathrm{O}$ & ${ }^{16} \mathrm{O}$ & ${ }^{17} \mathrm{O}$ & ${ }^{18} \mathrm{O}$ & ${ }^{19} \mathrm{O}$ & ${ }^{20} \mathrm{O}$ & \\
\hline 9 & & & & & & & & & ${ }^{17} \mathrm{~F}$ & ${ }^{18} \mathrm{~F}$ & ${ }^{19} \mathrm{~F}$ & ${ }^{20} \mathrm{~F}$ & & \\
\hline 10 & & & & & & & & & ${ }^{18} \mathrm{Ne}$ & ${ }^{19} \mathrm{Ne}$ & ${ }^{20} \mathrm{Ne}$ & ${ }^{21} \mathrm{Ne}$ & ${ }^{22} \mathrm{Ne}$ & ${ }^{23} \mathrm{Ne}$ \\
\hline 11 & & & & & & & & & & ${ }^{20} \mathrm{Na}$ & ${ }^{21} \mathrm{Na}$ & ${ }^{22} \mathrm{Na}$ & ${ }^{23} \mathrm{Na}$ & \\
\hline
\end{tabular}

Experimental uncertainties on $Y_{\mathrm{P}}$ are due to the ${ }^{1} \mathrm{H}(\mathrm{n}, \gamma){ }^{2} \mathrm{H}, \mathrm{D}(\mathrm{d}, \mathrm{n})^{3} \mathrm{He}$ and $\mathrm{D}(\mathrm{d}, \mathrm{p})^{3} \mathrm{H}$ reaction rates and to the neutron lifetime. For these three reactions, one finds (e.g. Coc et al., 2015. Cyburt, 2004)

$$
\Delta Y_{\mathrm{P}} \approx\left(1.5 \times 10^{-3}\right) \frac{\Delta\langle\sigma v\rangle}{\langle\sigma v\rangle},
$$

and $\Delta Y_{\mathrm{P}} \approx 0.18 \Delta \tau_{n} / \tau_{n}$ from Eq. (7). Since the experimental uncertainties are of the order of $10^{-2}$ for the rates and $10^{-3}$ for the lifetime, they may all contribute to the error budget.

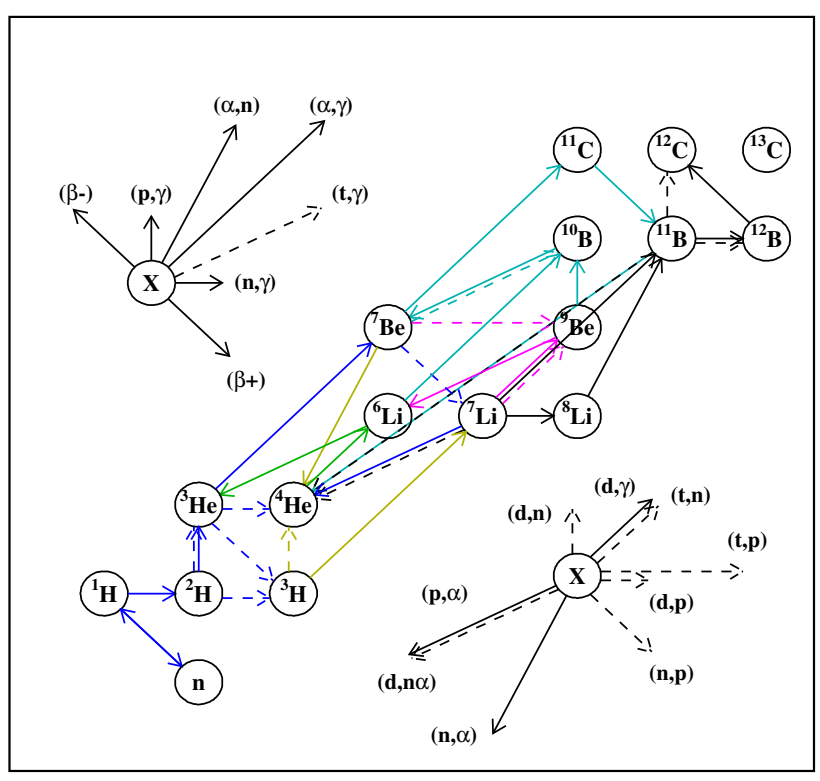

FIG. 22 Nuclear network of the most important reactions in BBN (out of the 424) up to ${ }^{7} \mathrm{Li}$ (blue), including ${ }^{6} \mathrm{Li}$ (green), ${ }^{10,11} \mathrm{~B}$ (light blue), ${ }^{9} \mathrm{Be}$ (pink) and up to $\mathrm{CNO}$ (black). The yellow arrows indicate the reactions that are now considered as unimportant.

There are basically two methods for the experimental determination of the neutron lifetime: detecting dying neutrons i.e. their decay rate ("beam experiments") or surviving neutrons after being left in a magnetic "bottle" for a certain time. Both methods produce slightly different results (see e.g. Fig. 8 in Young et al., 2014). This is most probably due to different systematic uncertainties but it might also be real and be explained by an undetected decay into a dark sector (see e.g. Fornal and Grinstein 2018). Note that in that case it would be the "bottle" results, corresponding to the surviving neutrons counting, that would be important for BBN. Hence, the value of the neutron lifetime has been revised several times by the Particle Data Group (PDG) from $885.7 \pm 0.8$ s (Amsler et al. 2008), used, e.g. in Coc and Vangioni (2010), to $879.4 \pm 0.6 \mathrm{~s}$ resulting from an average of "bottle" experiments (Czarnecki et al., 2018), slightly below the current PDG average (Patrignani and Particle Data Group, 2016 and 2017 update) which is $880.2 \pm 1.0 \mathrm{~s}$. We use the average over post-2000 experiments, $879.5 \pm 0.8 \mathrm{~s}$ (Serebrov et al. 2017$)$, which is extremely close to the average on bottle experiments, but with more conservative errors.

The cross section of the ${ }^{1} \mathrm{H}(\mathrm{n}, \gamma){ }^{2} \mathrm{H}$ reaction is obtained from calculations in the framework of Effective Field Theory whose results are estimated to be reliable to within $1 \%$ error (Ando et al. 2006). Indeed, the few experimental information available for this cross section at BBN energies are in very good agreement with theory (see Fig. 1 in $\mathrm{Coc}(2013)$ ). 
The $\mathrm{D}(\mathrm{p}, \gamma)^{3} \mathrm{He}, \mathrm{D}(\mathrm{d}, \mathrm{n})^{3} \mathrm{He}$ and $\mathrm{D}(\mathrm{d}, \mathrm{p})^{3} \mathrm{H}$ reactions are the main source of nuclear uncertainty for deuterium nucleosynthesis while the two last one may affect the error budget of $Y_{\mathrm{P}}$. The relative variations of $\mathrm{D} / \mathrm{H}$ are related to the variation of these rates (see e.g. Coc and Vangioni 2010) by

$$
\begin{aligned}
\frac{\Delta(\mathrm{D} / \mathrm{H})}{\mathrm{D} / \mathrm{H}} & =-0.32 \frac{\Delta\langle\sigma v\rangle_{\mathrm{d}(\mathrm{p}, \gamma)^{3} \mathrm{He}}}{\langle\sigma v\rangle_{\mathrm{d}(\mathrm{p}, \gamma)^{3} \mathrm{He}}} \\
\frac{\Delta(\mathrm{D} / \mathrm{H})}{\mathrm{D} / \mathrm{H}} & =-0.54 \frac{\Delta\langle\sigma v\rangle_{\mathrm{d}(\mathrm{d}, \mathrm{n})^{3} \mathrm{He}}}{\langle\sigma v\rangle_{\mathrm{d}(\mathrm{d}, \mathrm{n})^{3} \mathrm{He}}}-0.46 \frac{\Delta\langle\sigma v\rangle_{\mathrm{d}(\mathrm{d}, \mathrm{p})^{3} \mathrm{H}}}{\langle\sigma v\rangle_{\mathrm{d}(\mathrm{d}, \mathrm{p})^{3} \mathrm{H}}}
\end{aligned}
$$

so that to achieve the $\sim 1 \%$ precision required by observations, one needs a similar precision on reaction rates. None of them are affected by resonances, so that the only questions are to model the slowly varying energy dependence of the $S$-factors and precisely determine their absolute scale. There are basically two options: either empirically fit both the energy dependence and scale so as to follow closely the data, or use theoretical energy dependences from nuclear physics models and only determine the absolute normalization. We adopted the rates from the new evaluations of Iliadis et al. (2016) and Gómez Iñesta et al. (2017) that use both the second option, together with Bayesian methods. The theoretical, $a b$ initio energy dependences were taken from Marcucci et al. (2005) for $\mathrm{D}(\mathrm{p}, \gamma)^{3} \mathrm{He}$ and from Arai et al. (2011) for $\mathrm{D}(\mathrm{d}, \mathrm{n})^{3} \mathrm{He}$ and $\mathrm{D}(\mathrm{d}, \mathrm{p})^{3} \mathrm{H}$. The main difficulty to determine the absolute scale of the $S$-factors is that one needs to combine results from different experiments.

Coc et al. (2015), using traditional statistics found a normalization factor of $0.9900 \pm 0.0368$ for the $\mathrm{D}(\mathrm{p}, \gamma)^{3} \mathrm{He}$ theoretical $S$-factor of Marcucci et al. (2005), while the Bayesian analysis gives $1.000_{-0.036}^{+0.038}$ (Iliadis et al. 2016 ). This shows that, starting from the same experimental data and theoretical model, different statistical analyses can lead to a, significant, $1 \%$ difference. Figure 23 displays the $\mathrm{D}(\mathrm{p}, \gamma)^{3} \mathrm{He}$ experimental $S$-factor normalised to the theoretical model of Marcucci et al. (2005). The solid horizontal line corresponds to the scaling of the theoretical $S$-factor adopted by Coc et al. (2015). It is obvious that experimental data are scarce at BBN energies and slightly below the scaled $S$-factor (an overall $9 \%$ systematic uncertainty is not shown however), while the empirical fit by Adelberger et al. (2011) or (Descouvemont et al. 2004) follows closely, by construction, the experimental data. Note also that Marcucci et al. (2016) have included higher order terms in their ab initio model resulting in a $\approx 10 \%$ increase with respect with their previous result (Marcucci et al., 2005), this time well above the experimental data (see Fig. 23). Using this new theoretical $S$-factor, one would obtain an additional reduction of $\Delta(\mathrm{D} / \mathrm{H})=-0.072 \times 10^{-5}$ that nevertheless would vanish if we rescale it (by 0.915 ) to fit experimental data. This rate is thus a major source of uncertainty for $\mathrm{D} / \mathrm{H}$ prediction that should be resolved when the new experimental data (Gustavino, 2017) from LUNA at the Gran Sasso underground facility will be released, supplementing the low energy ones (Casella et al. 2002).

In a similar way, the $\mathrm{D}(\mathrm{d}, \mathrm{n})^{3} \mathrm{He}$ and $\mathrm{D}(\mathrm{d}, \mathrm{p})^{3} \mathrm{H}$ rates have been evaluated by (Coc et al. 2015) and later by (Gómez Iñesta et al., 2017), using the ab initio $S$-factor from Arai et al. (2011) scaled according to experimental data. They found negligible differences in scaling factors: $0.959 \pm 0.010$ and $0.955 \pm 0.010$ for the traditional analysis to be compared with $0.961 \pm 0.010$ and $0.956 \pm 0.010$ for the Bayesian one. However, the theoretical work of Arai et al. (2011) was focused on low energies and does not correctly reproduce the $\mathrm{D}(\mathrm{d}, \mathrm{n})^{3} \mathrm{He}$ and $\mathrm{D}(\mathrm{d}, \mathrm{p})^{3} \mathrm{H}$ experimental data above $\approx 600 \mathrm{keV}$. It is highly desirable that these calculations be extended up to $\approx 2 \mathrm{MeV}$, to cover the range of experimental data that encompass BBN energies.

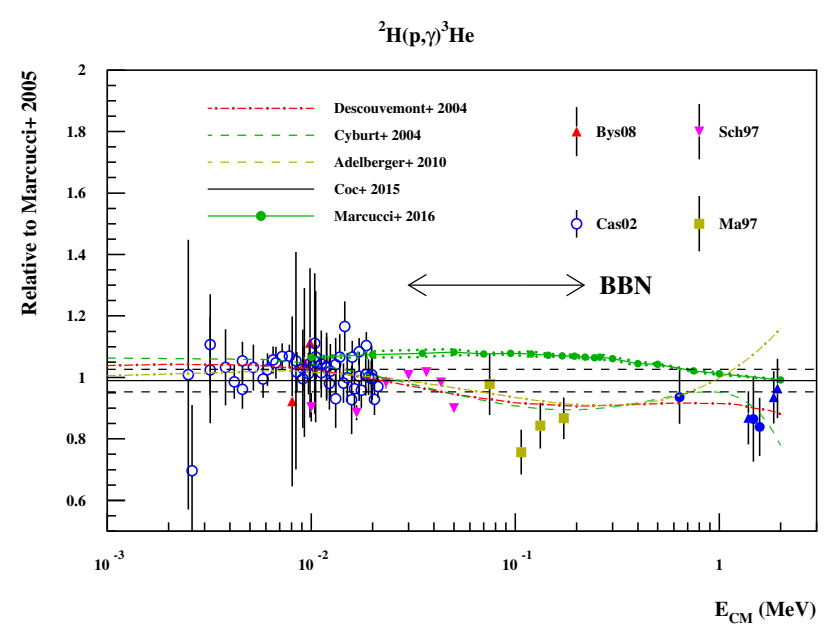

FIG. 23 Ratio of experimental (Bystritsky et al. 2008 Casella et al., 2002, Ma et al., 1997; Schmid et al., 1997), fitted (Adelberger et al. | 2011, Coc et al. | 2015 | Cyburt, 2004, Descouvemont et al. , 2004) and new theoretical (Marcucci et al. 2016) $S$-factors to the theoretical one (Marcucci et al. 2005); the horizontal lines correspond to the theoretical $S$ factor scaled according to Coc et al. (2015). (Systematic uncertainties, i.e. global normalization errors, in the range $4.5-9 \%$ are shown in keys).

At the CMB deduced density, ${ }^{7} \mathrm{Li}$ is produced through the formation of ${ }^{7} \mathrm{Be}$ via the ${ }^{3} \mathrm{He}(\alpha, \gamma){ }^{7} \mathrm{Be}$ reaction as ${ }^{7} \mathrm{Be}$ will decay much later to ${ }^{7} \mathrm{Li}$. The destruction of ${ }^{7} \mathrm{Be}$ occurs through the ${ }^{7} \mathrm{Be}(\mathrm{n}, \mathrm{p})^{7} \mathrm{Li}(\mathrm{p}, \alpha)^{4} \mathrm{He}$ channel which is limited by the scarcity of late time neutron abundance. The most influential reaction rates on ${ }^{7} \mathrm{Li}$ nucleosynthesis are (e.g. Table 1 in Coc and Vangioni, 2010) ${ }^{1} \mathrm{H}(\mathrm{n}, \gamma){ }^{2} \mathrm{H}$ (indirectly by affecting the neutron abundance) and ${ }^{3} \mathrm{He}(\alpha, \gamma){ }^{7} \mathrm{Be}$, but large deviations from their nominal cross sections are strongly constrained by experiments. Even though, there has not been new experimental data, since it is the major source of uncertainty on the ${ }^{7} \mathrm{Li}$ production, the ${ }^{3} \mathrm{He}(\alpha, \gamma){ }^{7} \mathrm{Be}$ reaction rate has also been recently re-evaluated using Bayesian methods (Gómez Iñesta et al. 2017) to scale the theoretical $S_{-}$ 
factor of Neff (2011). It was known that the ${ }^{7} \mathrm{Be}(\mathrm{n}, \alpha)^{4} \mathrm{He}$ reaction could not help solve the lithium problem, but its rate was highly uncertain and affected the ${ }^{7} \mathrm{Li}$ production at the few percent level. Until recently, the only published rate came from an evaluation by Wagoner (1969) based on very scarce data. We used either this rate or the one obtained by TALYS (Goriely et al. 2008) in previous publications (Coc et al., 2012, Coc et al. | 2014). A new re-evaluation (Hou et al., 2015$)$ and experiments (Barbagallo et al. 2016 Kawabata et al., 2017) confirmed that the ${ }^{7} \mathrm{Be}(\mathrm{n}, \alpha){ }^{4} \mathrm{He}$ rate is approximately, one order of magnitude below the Wagoner one, rendering negligible the effect this reaction. Hence, we now use the rate provided by the $\mathrm{n}$ TOF collaboration (Barbagallo et al. 2016$)$ that now has no impact on ${ }^{7} \mathrm{Be}$.

Lithium-6 nucleosynthesis is quite simple given that it is only produced by the $\mathrm{D}(\alpha, \gamma){ }^{6} \mathrm{Li}$ reaction and destroyed by ${ }^{6} \operatorname{Li}(p, \alpha){ }^{4} \mathrm{He}$. While the rate of the latter has been precisely known for a long time, the rate of the former suffered from large uncertainties Angulo et al. 1999). This has now been solved, thanks to experiments (Anders et al., 2014, Hammache et al., 2010), and theory (Mukhamedzhanov et al., 2016).

Elements with atomic number above 7 are not expected to be significantly produced in BBN, unless some of the reaction rates involved in their production differ strongly from their current estimates (Coc et al. 2014: Iocco et al. 2007). Indeed, some of them rely on theoretical models not well adapted to low mass nuclei. Figure 22 displays the main reactions producing or destructing the beryllium, boron and $\mathrm{C}, \mathrm{N}$ and $\mathrm{O}$ stable isotopes.

Table IV displays the few reaction rates that have been updated in Coc et al. (2015, 2014), and now in this work, with respect to the Table 4 of Coc et al. (2012).

TABLE IV Updated reaction rates with respect to Coc et al. (2012)

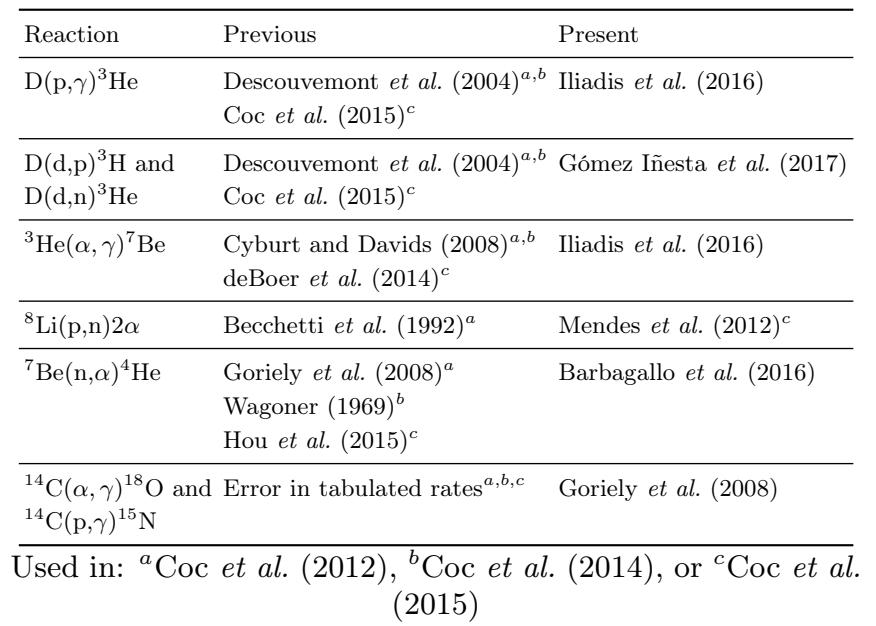

\section{NUMERICAL RESULTS}

\section{A. Overview of PRIMAT}

To our knowledge, apart from the Kawano code (Kawano, 1992) which is based on the historical code of Wagoner (Wagoner, 1969, 1973; Wagoner et al., 1967), there exist only two public $\mathrm{BBN}$ codes, which are PArthENoPE (Consiglio et al. 2017; Pisanti et al., 2008) and AlterBBN (Arbey, 2012). The method followed in PRIMAT differs slightly from these two recent implementations, in that we integrate directly differential equations in time, instead of integrating differential equations in the plasma temperature which are obtained by the replacement of $\mathrm{d} T / \mathrm{d} t$ derived from the Friedmann equation. The code is abundantly commented and refers to equations of the previous sections of this article. Let us summarize the main steps of the code.

- First we solve for the thermodynamics of the plasma following the details provided in $\S$ II . This allows to obtain $a(T)$ either using entropy conservation (31) if the effect of incomplete neutrino decoupling is neglected, or using the variation of entropy from the heat transfer between the plasma and the neutrinos 62. When QED plasma effects are included we use the corresponding (55). The relation $T(a)$ is obtained by a numerical inversion. The temperature of neutrinos is either deduced from (29), or (57) when QED plasma effects are included, if we assume they are fully decoupled. If we take into account the incomplete neutrino decoupling, then their effective temperature is obtained from Eqs. 63 and (64). The evolution of baryon energy density follows the simple scaling (34), and similarly for cold dark matter. Then the cosmological expansion is solved using the total energy density (43) [with QED effects included for the plasma energy density (Eq. 58) if the choice is made], inside the Friedmann equation (40), so to obtain $a(t)$ by numerical resolution of the differential equation. The relation $t(a)$ is obtained by numerical inversion. Eventually we obtain $T(t)$ as $T(a(t))$ that we can use later in the reaction rates since they depend on temperature.

- Once the thermodynamics and the cosmological expansion are known, we compute the weak rates for a grid of plasma temperatures so as to interpolate them. The total rates are obtained by considering all relevant corrections discussed in $\S$ III. which are added as in Eq. 121). The constant $K$ involved in all rates is obtained from Eq. (91) with $\lambda_{0}$ given by the value 120 . Since the weak rates computation can be very long, especially when including finite temperature radiative corrections which involve two-dimensional integrals, we store them on hard disk for later use. An option allows to recompute them when desired and various options allow 
to switch on or off the various corrections.

- Finally we build the system of equations for the nuclear network, that is the system of Eqs. 138 including the weak rates. The nuclear reaction rates are read from tabulated values in function of temperature, or from analytical fits and the reverse rates are obtained from (141). The evolution of the nuclides abundances is numerically solved in three periods.

1. For $10^{11} \mathrm{~K} \geq T \geq 10^{10} \mathrm{~K}$ we solve only for the abundance of neutrons and protons and totally ignore the nuclear reactions. Photodissociation reactions are too strong for this period and only the abundance of neutrons and protons is relevant at this stage. Nuclear reactions are anyway not tabulated above $10^{10} \mathrm{~K}$.

2. Then for $10^{10} \mathrm{~K} \geq T \geq 1.25 \times 10^{9} \mathrm{~K}$ we solve for a small nuclear network, made of light elements only (protons, neutron, ${ }^{2} \mathrm{H},{ }^{3} \mathrm{H},{ }^{3} \mathrm{He}$, ${ }^{4} \mathrm{He},{ }^{6} \mathrm{Li},{ }^{7} \mathrm{Li}$ and ${ }^{7} \mathrm{Be}$ ) and starting from the NSE abundance, apart for neutrons and protons which are taken from the previously solved period. For this period the system is very stiff and we use a first order BDF scheme (backward differentiation formula), which is equivalent to a backward Euler method.

3. Finally for $1.25 \times 10^{9} \mathrm{~K} \geq T \geq 6 \times 10^{7} \mathrm{~K}$ we solve numerically for all nuclides, using the full network of reactions. The system is less stiff and it is possible to fasten the numerical integration by using a second order BDF scheme.

\section{B. Temperature of nucleosynthesis}

One can estimate the temperature of nucleosynthesis as the temperature for which the NSE value of deuterium is equal to the abundance of neutrons found from the simple freeze-out plus beta decay model (98). At this temperature deuterium would have gobbled up all free neutrons and this definition corresponds essentially to the end of nucleosynthesis. Hence $T_{\mathrm{Nuc}}$ is defined by

$$
Y_{n}\left(T_{\mathrm{Nuc}}\right) \equiv Y_{d}^{\mathrm{NSE}}\left(T_{\mathrm{Nuc}}\right)
$$

These two abundances are plotted in Fig. 24 and we find numerically $T_{\mathrm{Nuc}} \simeq 7.7 \times 10^{8} \mathrm{~K} \simeq 0.066 \mathrm{MeV}$ and a corresponding $t_{\mathrm{Nuc}} \simeq 300 \mathrm{~s}$. On Fig. 3, we see that nearly all neutrons are hidden in ${ }^{4} \mathrm{He}$ which is energetically more favored than deuterium. Overall the deuterium is only a catalytic agent, necessary to convert free neutrons in ${ }^{4} \mathrm{He}$, and we find only traces of it at the end of BBN. The nucleosynthesis of all rare light elements is over when the temperature is around $6 \times 10^{7} \mathrm{~K}$, corresponding to $t_{\text {end }} \simeq 5 \times 10^{4} \mathrm{~s}$. In our code, the decay reactions of elements whose half-life is much longer than $t_{\text {end }}$ are not included. However when reporting the final abundances of elements, it is customary to consider that these elements have been fully converted into stable elements. For instance the half-life of ${ }^{7} \mathrm{Be}$ is 53.22 days, as it decays into ${ }^{7} \mathrm{Li}$. Hence when reporting the latter abundances, we add the former ones. Similarly, tritium $\left({ }^{3} \mathrm{H}\right)$ decays in ${ }^{3} \mathrm{He}$ in 12.32 years and the former is added to the latter in the final abundances reported. The evolution of the isotopes as a function of time is depicted in Fig. $25^{18}$.

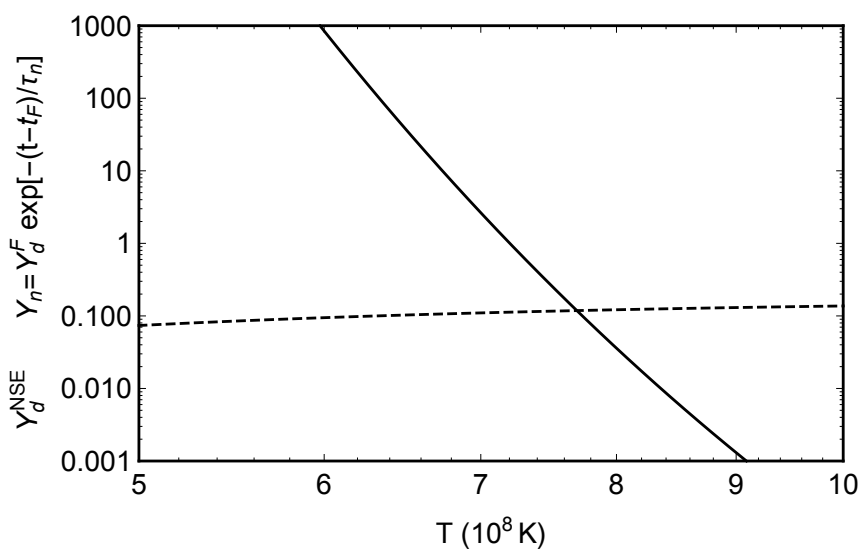

FIG. 24 Evolution of NSE deuterium abundance (continuous line) and $Y_{n}^{F}$ found from a simple freeze-out and beta decay model (dashed line).

\section{Effect of corrections on abundances}

The effects of the various corrections in the weak rates have been estimated numerically and are reported in table V] We have assessed the importance of individual corrections as well as the interplay of some corrections when they do not add simply linearly. Let us now comment in details the effect of these corrections.

\section{Radiative corrections}

Using the non-relativistic Fermi function and Sirlin's function B30 without resummation of higher order corrections, we find $\Delta Y_{\mathrm{P}}^{(4)}=31.0$, exactly as in Lopez and Turner (1999, Table V, line 2). With the resummed radiative corrections (B35) and using the relativistic Fermi function brings an extra $\Delta Y_{\mathrm{P}}^{(4)}=0.5$, worth being considered for precise predictions.

\footnotetext{
18 The time evolution of ${ }^{14} \mathrm{C}$ in Fig. 25 left panel, strongly differs from the one in Fig. 13 of Coc et al. (2012). This was due to an error in the ${ }^{14} \mathrm{C}(\alpha, \gamma){ }^{18} \mathrm{O}$ and ${ }^{14} \mathrm{C}(\mathrm{p}, \gamma)^{10} \mathrm{~N}$ reaction rates that are now fixed. It has, however no consequences on the total CNO production.
} 

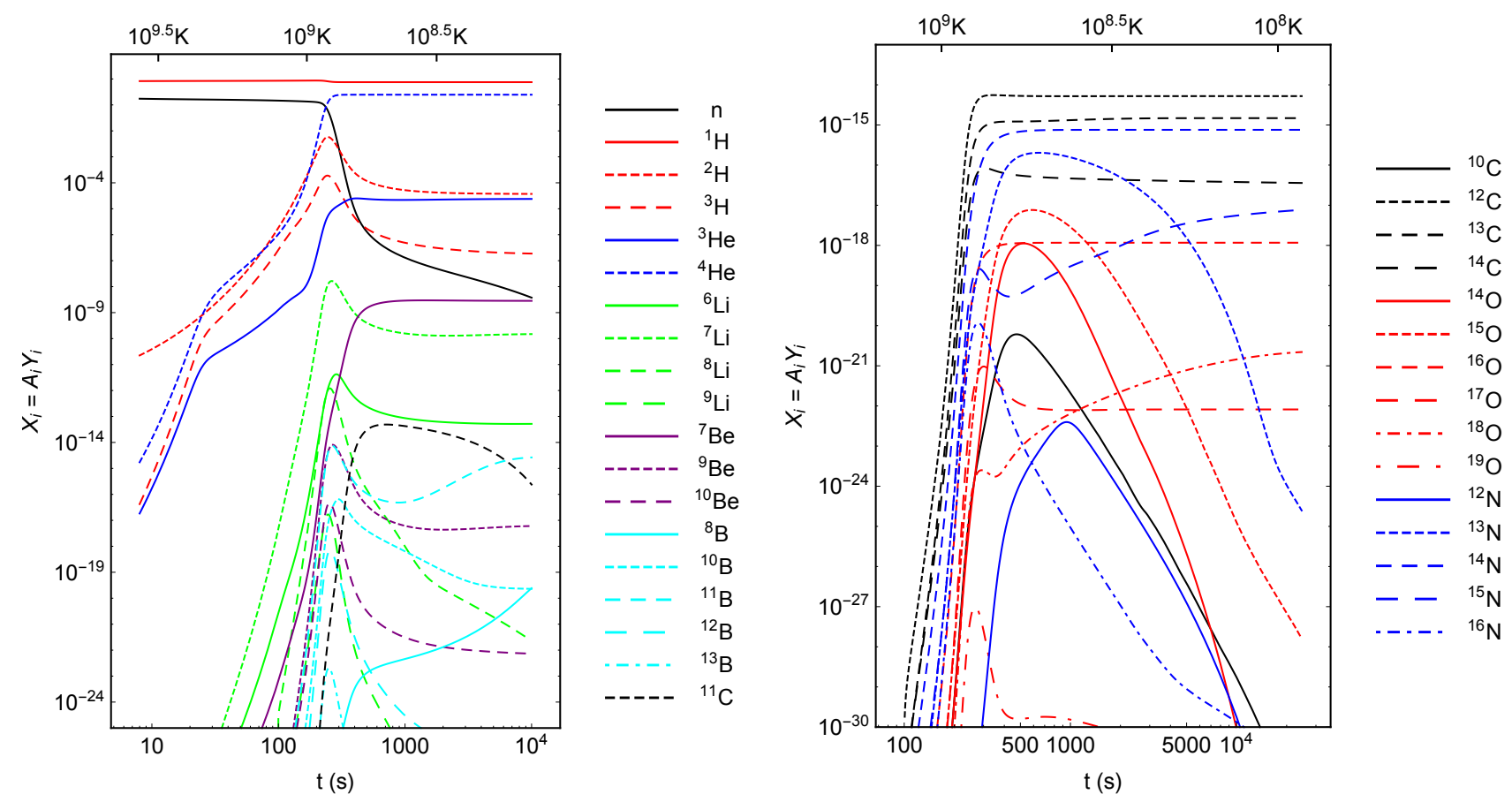

FIG. 25 Left: Evolution of light elements abundances. Right: Evolution of heavier elements abundances ${ }^{18}$.

\section{Finite nucleon mass corrections}

We find that the cumulated effect of finite nucleon mass corrections and weak-magnetism brings $\Delta Y_{\mathrm{P}}^{(4)}=12.8$ corresponding to $+0.53 \%$ This is slightly more than found by Lopez and Turner (1999, Table V, line 3) which is $\Delta Y_{\mathrm{P}}^{(4)}=12$ corresponding to $+0.50 \%$, also evoked in Lopez et al. (1997). Given the smallness of the difference we consider that our results are in agreement with these references. Note that weak-magnetism accounts for around $12 \%$ of the finite nucleon mass corrections.

It is worth commenting that when finite nucleon mass effects are considered together with incomplete neutrino decoupling, the total effect is less than the sum of the effects taken individually and there is a reduction $\Delta Y_{\mathrm{P}}^{(4)} \simeq$ -0.6 . We found that this is because finite nucleon mass corrections are very sensitive to the neutrino temperature (see Fig. 20). Hence we advocate that a correct treatment of neutrino decoupling needs not only to be performed with the full machinery of the Boltzmann equation, with the inclusion of neutrino oscillations, but it should also be performed jointly with the inclusion of finite nucleon mass corrections. So far this joint treatment is lacking in the literature.

\section{Finite temperature radiative corrections}

In table $\mathrm{V}$, we report that the finite radiative corrections bring $\Delta Y_{\mathrm{P}}^{(4)} \simeq 3.1$, reduced to $\Delta Y_{\mathrm{P}}^{(4)} \simeq 0$ when bremsstrahlung corrections are added to obtain a consistent detailed balance of weak rates. Indeed in Fig. (18) we check that around freeze-out, finite radiative corrections and bremsstrahlung corrections are almost opposite. In both cases this is very different from the modification computed in Esposito et al. (2000a) where $\Delta Y_{\mathrm{P}}^{(4)} \simeq-4$ is reported.

It is important to realize that the electron mass shift (44) is part of the finite-temperature corrections when it comes to considering the corrections to the weak rates. Hence we adopt the point of view of Brown and Sawyer (2001) which is different from Lopez and Turner (1999). In order to allow a comparison with that reference, we evaluated independently the effect of the electron mass shift following their method, that is replacing the electron mass shift directly in the distribution functions, and we found a modification $\Delta Y_{\mathrm{P}}^{(4)} \simeq+1.4$. Hence from the estimation of Lopez and Turner (1999) for finitetemperature radiative corrections $\Delta Y_{\mathrm{P}}^{(4)} \simeq 3$ one should instead use $\Delta Y_{\mathrm{P}}^{(4)} \simeq 4.4$ to compare with our results. This is slightly larger than our value $\Delta Y_{\mathrm{P}}^{(4)} \simeq 3.1$ without BS corrections but certainly larger than our value $\Delta Y_{\mathrm{P}}^{(4)} \simeq 0$ with BS corrections.

In general, an enhancement of the rates induces a decrease of $Y_{\mathrm{P}}$ thanks to Eq. (7). In Lopez and Turner (1999) the rates are increased by the finite temperature radiative corrections, and one would expect a decrease $Y_{\mathrm{P}}$. However since the corrected rates no longer satisfy the detailed balance relation (86) as they should, Eq. (7) 
TABLE V Final abundances depending on the corrections included. ID is incomplete decoupling of neutrinos. FM is finite nucleon mass effect without weak-magnetism, WM is weak-magnetism, and FM+WM are both effects. RC are radiative corrections. ThRC are finite temperature radiative corrections without bremsstrahlung corrections, and BS are bremsstrahlung corrections. QED-MS is the QED electron mass shift effect considered alone when replaced directly in distribution functions (see discussion in $\$$ V.C.3), and QED-Pl are the QED effects on the plasma thermodynamics (\$I.E).

\begin{tabular}{|c|c|c|c|c|c|c|c|}
\hline Corrections & $Y_{\mathrm{P}}$ & $\delta Y_{\mathrm{P}} \times 10^{4}$ & $\delta Y_{\mathrm{P}} / Y_{\mathrm{P}}(\%)$ & $\mathrm{D} / \mathrm{H} \times 10$ & $\Delta(\mathrm{D} / \mathrm{H})$ & $\mathrm{He} / \mathrm{H} \times$ & $\mathrm{i} / \mathrm{H} \times 10^{10}$ \\
\hline Born & 0.24262 & 0 & 0 & 2.423 & 0 & 1.069 & 5.635 \\
\hline Born+ID & 0.24274 & 1.2 & 0.05 & 2.432 & 0.37 & 1.070 & 5.613 \\
\hline Born+FM & 0.24374 & 11.2 & 0.46 & 2.430 & 0.25 & 1.070 & 5.651 \\
\hline Born $+\mathrm{FM}+\mathrm{WM}$ & 0.24390 & 12.8 & 0.53 & 2.430 & 0.29 & 1.070 & 5.654 \\
\hline RCa [Eq. B30, Non. Rel. Fermi] & 0.24572 & 31.0 & 1.27 & 2.440 & 0.70 & 1.071 & 5.681 \\
\hline RCb [Eq. $\sqrt{\text { B35 }}$, Non. Rel. Fermi] & 0.24575 & 31.3 & 1.29 & 2.440 & 0.70 & 1.071 & 5.682 \\
\hline RC [Eq. B35, Rel. Fermi] & 0.24577 & 31.5 & 1.30 & 2.440 & 0.70 & 1.071 & 5.682 \\
\hline $\mathrm{RC}+\mathrm{QED}-\mathrm{MS}$ & 0.24591 & 32.9 & 1.36 & 2.441 & 0.74 & 1.071 & 5.684 \\
\hline $\mathrm{RC}+\mathrm{QED}-\mathrm{Pl}$ & 0.24577 & 31.5 & 1.30 & 2.443 & 0.82 & 1.072 & 5.674 \\
\hline $\mathrm{RC}+\mathrm{ID}$ & 0.24588 & 32.6 & 1.34 & 2.449 & 1.07 & 1.073 & 5.660 \\
\hline $\mathrm{RC}+\mathrm{ID}+\mathrm{QED}-\mathrm{Pl}$ & 0.24588 & 32.6 & 1.34 & 2.452 & 1.19 & 1.073 & 5.652 \\
\hline $\mathrm{RC}+\mathrm{FM}+\mathrm{WM}$ & 0.24705 & 44.3 & 1.82 & 2.447 & 0.99 & 1.072 & 5.701 \\
\hline $\mathrm{RC}+\mathrm{FM}+\mathrm{WM}+\mathrm{QED}-\mathrm{MS}$ & 0.24718 & 45.6 & 1.87 & 2.448 & 1.03 & 1.073 & 5.701 \\
\hline $\mathrm{RC}+\mathrm{FM}+\mathrm{WM}+\mathrm{QED}-\mathrm{Pl}$ & 0.24704 & 44.2 & 1.81 & 2.450 & 1.11 & 1.073 & 5.693 \\
\hline $\mathrm{RC}+\mathrm{FM}+\mathrm{WM}+\mathrm{ID}$ & 0.24720 & 44.8 & 1.84 & 2.456 & 1.36 & 1.074 & 5.678 \\
\hline $\mathrm{RC}+\mathrm{FM}+\mathrm{WM}+\mathrm{ThRC}($ No BS$)$ & 0.24736 & 47.4 & 1.95 & 2.449 & 1.07 & 1.073 & 5.706 \\
\hline $\mathrm{RC}+\mathrm{FM}+\mathrm{WM}+\mathrm{ThRC}+\mathrm{BS}$ & 0.24705 & 44.3 & 1.82 & 2.447 & 0.99 & 1.072 & 5.701 \\
\hline $\mathrm{RC}+\mathrm{FM}+\mathrm{WM}+\mathrm{ThRC}+\mathrm{BS}+\mathrm{ID}+\mathrm{QED}-\mathrm{Pl}$ & 0.24709 & 44.7 & 1.84 & 2.459 & 1.49 & 1.074 & 5.670 \\
\hline
\end{tabular}

cannot be used and the effect is not opposite. In our case this can be seen when using the radiative corrections, without the BS corrections added. The weak rates are increased (see Fig. 18) and still it leads to $\Delta Y_{\mathrm{P}}^{(4)} \simeq 3.1$. This highlights the extreme importance of constructing corrections which respect the detailed balance, in order to obtain meaningful results. Any corrections added which does not satisfy the correct detailed balance relation Eq. (7) is somehow equivalent to a modification of the value of the mass gap $\Delta$, modifying artificially the thermal equilibrium value. Stated more directly, it is obvious that an overestimated enhancement of $\Gamma_{p \rightarrow n}$ as in Lopez and Turner (1999) leads to an artificial increase of $Y_{n}$ and thus of $Y_{\mathrm{P}}$. When a correction does not satisfy the detailed balance relation, the primary effect is no more a delayed or advanced freeze-out, that is a lower or larger freeze-out temperature, but an artificially different freeze-out abundance for the same freeze-out temperature since one rate is overestimated and the thermal equilibrium is artificially displaced.

\section{QED effects on plasma thermodynamics}

The effect of QED corrections on $Y_{\mathrm{P}}$ is negligible. In Lopez and Turner (1999), it is estimated to be around $\Delta Y_{\mathrm{P}}^{(4)} \simeq 1$ only because it is also cumulated with the electron mass shift effects which are part of finite temperature corrections of the weak rates in our terminology. We find $\Delta Y_{\mathrm{P}}^{(4)} \simeq 1.4$ when computing it with the same method.

\section{Incomplete neutrino decoupling}

The pure clock effect evoked at the end of $\S$ III.I is not the only effect, because one must also consider the effect of incomplete neutrino decoupling on the weak rates. In our approximate description, we have implicitly assumed in $\S$ II.G that all neutrino flavors share the same ratio of heating, that is we assume that $\delta \rho_{\nu_{e}} / \rho_{\nu_{e}}=\delta \rho_{\nu_{\mu}} / \rho_{\nu_{\mu}}=$ $\delta \rho_{\nu_{\tau}} / \rho_{\nu_{\tau}}$ so that it is meaningful to define a common effective temperature. It is certainly not correct since there is more energy gained by electronic neutrino than other types of neutrino. Indeed electronic neutrinos couple to electrons and positron with charged and neutral currents whereas the other flavors of neutrinos couple only through neutral currents. However, first this is less the case when considering neutrino flavor mixing (Mangano et al. , 2005), and second in the early stage of neutrino heating by electron-positron annihilations, the heating is more efficiently redistributed among the three species. Hence it is not a too bad approximation to assume that the heating is distributed in the same ratio among flavors when considering the effect on weak rates. This means that we assume that all neutrino flavors share always $1 / N_{\nu}$ of the total energy density. Even though we know that they have distorted spectra, we still defined an effective neutrino temperature from their energy density. That is we still use Eq. 42 to define the neutrino tem- 
perature $T_{\nu}$, and by construction it is the temperature for thermalized neutrino distributions that would have the same energy density.

Apart from the clock effect mentioned earlier, there are two competing effects in the weak rates which nearly fully cancel (Dodelson and Turner, 1992, Fields et al. 1993. Mangano et al. 2005). First the higher energy density in neutrinos results in an increase of the weak rates, inducing a freeze-out which happens later, with less neutrons and thus producing less ${ }^{4} \mathrm{He}$. However the energy gained by neutrinos is lost from the plasma, and the reduction in electrons-positrons energy density results in lower weak rates, inducing an earlier freeze-out which results in more neutrons and then more ${ }^{4} \mathrm{He}$ production. It has been shown in Fields et al. (1993) that both effects level off when the freeze out is complete, that is around $T=3.3 \times 10^{9} \mathrm{~K}$. Indeed we find that in our simple thermal approximation, the relative variation in the neutron fraction at that temperature is a modest relative increase of $2 \times 10^{-5}$. Without the clock effect this would result in a negligible $\Delta Y_{\mathrm{P}}^{(4)} \simeq 0.05$. It is only the clock effect (see $\S$ V.C., that is the fact that neutrons have slightly less time to beta decay, which results in $\Delta Y_{\mathrm{P}}^{(4)} \simeq 1.2$. When taking more carefully into account the fact that neutrinos do not get the same share of the heating, and that furthermore there are spectral distortions which affect the neutrino distribution functions entering the weak-rates, it is found a slightly larger increase in $Y_{\mathrm{P}}^{(4)}$ Mangano et al. 2005).

However note that our variations for $Y_{\mathrm{P}}, \mathrm{D} / \mathrm{H},{ }^{3} \mathrm{He} / \mathrm{H}$ and ${ }^{7} \mathrm{Li} / \mathrm{H}$ are in very close agreement with those found in Grohs et al. (2016) (Table IV, second line), where these spectral distortions effects (but not the flavor oscillations) have been fully taken into account. It is puzzling that with our thermal approximation based on a heating rate found from Pisanti et al. (2008), that is from the result of Mangano et al. $[(2005)$, we do not recover the results of table 3 in Mangano et al. (2005) but we recover with very good agreement the results of Grohs et al. (2016). We found that the argument presented in Grohs et al. (2016) for the variations of $\mathrm{D} / \mathrm{H},{ }^{3} \mathrm{He} / \mathrm{H}$ and ${ }^{7} \mathrm{Li} / \mathrm{H}$ are very convincing and we recover them in our numerics. Indeed the clock effect results in less time to destroy deuterium through $\mathrm{D}(\mathrm{d}, \mathrm{n})^{3} \mathrm{He}, \mathrm{D}(\mathrm{p}, \gamma)^{3} \mathrm{He}$ and $\mathrm{D}(\mathrm{d}, \mathrm{p})^{3} \mathrm{H}$, ending up in more deuterium being left out at the end of BBN. Since two of these deuterium destroying reactions are producing ${ }^{3} \mathrm{He}$ [namely $\mathrm{D}(\mathrm{d}, \mathrm{n})^{3} \mathrm{He}$ and $\mathrm{D}(\mathrm{p}, \gamma)^{3} \mathrm{He}$, this results in more ${ }^{3} \mathrm{He}$. As for ${ }^{7} \mathrm{Li}$ it is reduced only because the production of ${ }^{7} \mathrm{Be}$ has less time to proceed, but we found that the ${ }^{7} \mathrm{Li}$ (without ${ }^{7} \mathrm{Be}$ added) is increased since it has also less time to be destroyed down from its peak value during $\mathrm{BBN}$. Hence the signs of variations for $\mathrm{D} / \mathrm{H},{ }^{3} \mathrm{He} / \mathrm{H}$ and ${ }^{7} \mathrm{Li} / \mathrm{H}$ have a clear physical origin, when incomplete neutrino decoupling is taken into account, and these signs are opposite to those reported in table 3 of Mangano et al. (2005).
TABLE VI First coefficients of Eq. 145. These provide abundances with precision better than $0.01 \%$ for $Y_{\mathrm{P}}$ and $0.03 \%$ for other abundances, in the range of $10 \%$ variations in $\Omega_{\mathrm{b}} h^{2}, 2 \%$ variation in $\tau_{\nu}$ and $2 \leq N_{\nu} \leq 4$. However these are still subject to reaction rates uncertainties which are estimated below in table VII

\begin{tabular}{lrrrr}
\hline \multicolumn{1}{c}{$Y_{\mathrm{P}}$} & \multicolumn{1}{c}{$\mathrm{D} / \mathrm{H}$} & ${ }^{3} \mathrm{He} / \mathrm{H}$ & ${ }^{7} \mathrm{Li} / \mathrm{H}$ \\
\hline$C_{100}$ & 0.039039 & -1.64550 & -0.56699 & 2.07605 \\
$C_{010}$ & 0.163552 & 0.40901 & 0.13587 & -0.27675 \\
\hline$C_{110}$ & -0.000044 & -0.61229 & -0.12157 & -0.29277 \\
$C_{200}$ & -0.029351 & 2.04137 & 0.53303 & 0.58639 \\
$C_{020}$ & -0.036124 & -0.00599 & -0.01265 & 0.03888 \\
\hline$C_{300}$ & 0.017891 & -2.40817 & -0.51855 & -0.88243 \\
$C_{210}$ & -0.001037 & 0.80150 & 0.12083 & 0.51082 \\
$C_{120}$ & -0.000354 & -0.00477 & 0.00928 & -0.10335 \\
$C_{030}$ & 0.009938 & 0.00224 & 0.00270 & 0.00884 \\
\hline \hline$C_{001}$ & 0.731614 & 0.42220 & 0.14052 & 0.43829 \\
$C_{101}$ & -0.009741 & -0.66030 & -0.12390 & 1.22619 \\
$C_{011}$ & 0.018321 & 0.19366 & 0.04040 & -0.33069 \\
$C_{111}$ & -0.003423 & -0.33158 & -0.03879 & -0.70923 \\
$C_{201}$ & 0.004189 & 0.90617 & 0.12121 & 0.92463 \\
$C_{021}$ & -0.011981 & 0.00498 & -0.00328 & 0.13530 \\
\hline & & & &
\end{tabular}

\section{Dependence on main parameters}

The variations of the yields for small variations of the main parameters can be given with expansions of the type

$$
\frac{\Delta Y_{\mathrm{P}}}{\overline{Y_{\mathrm{P}}}}=\sum_{p q r} C_{p q r}\left(\frac{\Delta \Omega_{\mathrm{b}} h^{2}}{\overline{\Omega_{\mathrm{b}} h^{2}}}\right)^{p}\left(\frac{\Delta N_{\nu}}{\overline{N_{\nu}}}\right)^{q}\left(\frac{\Delta \tau_{n}}{\overline{\tau_{n}}}\right)^{r}
$$

with similar expansions for the other abundances $\left(\mathrm{D} / \mathrm{H},{ }^{3} \mathrm{He} / \mathrm{H},{ }^{7} \mathrm{Li} / \mathrm{H}\right)$. The reference abundances are given by the last line of table $\mathrm{V}$ and the reference parameters are $\overline{\Omega_{\mathrm{b}} h^{2}}=0.02225, \overline{\tau_{n}}=879.5 \mathrm{~s}$ and $\overline{N_{\nu}}=3$. The meaning of varying the number of neutrinos is further discussed in $\S$ VI.D. The first coefficients of these expansions are given in table $\mathrm{VI}$

Leaving aside the non-standard BBN physics which corresponds to a variation of the number of neutrinos, we can restrict this expansion to the linear behaviour to estimate rapidly the variations of the abundances in function of changes in the baryon abundance or the neutron lifetime. Hence if these parameters are slightly modified in the future, our results can be transposed easily. We 
find

$$
\begin{aligned}
\frac{\Delta Y_{\mathrm{P}}}{\overline{Y_{\mathrm{P}}}} & =0.0390 \frac{\Delta \Omega_{\mathrm{b}} h^{2}}{\overline{\Omega_{\mathrm{b}} h^{2}}}+0.732 \frac{\Delta \tau_{n}}{\overline{\tau_{n}}} \\
\frac{\Delta \mathrm{D} / \mathrm{H}}{\overline{\mathrm{D} / \mathrm{H}}} & =-1.65 \frac{\Delta \Omega_{\mathrm{b}} h^{2}}{\overline{\Omega_{\mathrm{b}} h^{2}}}+0.422 \frac{\Delta \tau_{n}}{\overline{\tau_{n}}} \\
\frac{\Delta^{3} \mathrm{He} / \mathrm{H}}{\overline{{ }^{3} \mathrm{He} / \mathrm{H}}} & =-0.567 \frac{\Delta \Omega_{\mathrm{b}} h^{2}}{\overline{\Omega_{\mathrm{b}} h^{2}}}+0.141 \frac{\Delta \tau_{n}}{\overline{\tau_{n}}} \\
\frac{\Delta^{7} \mathrm{Li} / \mathrm{H}}{{ }^{7} \mathrm{Li} / \mathrm{H}} & =2.08 \frac{\Delta \Omega_{\mathrm{b}} h^{2}}{\overline{\Omega_{\mathrm{b}} h^{2}}}+0.438 \frac{\Delta \tau_{n}}{\overline{\tau_{n}}}
\end{aligned}
$$

As expected, $Y_{\mathrm{P}}$ is very sensitive to the weak rates and thus to $\tau_{n}$ but not to the baryon abundance. Hence even though uncertainty is larger in baryon abundance, the theoretical uncertainty of $Y_{\mathrm{P}}$ is dominated by the uncertainty in the determination of the neutron lifetime. From the measured value of $\tau_{n}$ reported in appendix $\mathrm{D}$ this corresponds to a $0.068 \%$ uncertainty in $Y_{\mathrm{P}}$ or $\sigma\left(Y_{\mathrm{P}}\right) \simeq 0.00017$ which is lower than the value $\sigma\left(Y_{\mathrm{P}}\right) \simeq 0.0003$ used in Ade et al. (2016, p47), implying that we find errors approximately $40 \%$ smaller in $Y_{\mathrm{P}}$. The predicted and observed abundances as functions of $\eta$ are plotted in Fig. 26

\section{E. Distribution of abundance predictions}

We use the method described in Coc et al. (2014) to estimate the uncertainty in light elements productions during $\mathrm{BBN}$ due to uncertainty in nuclear rates and weakrates (that is the uncertainty on $\tau_{n}$ that we assume to follow a normal distribution). It has been found (Longland et al. 2010), that probability density functions of reaction rates can be well approximated by lognormal distributions

$$
f(x)=\frac{1}{\sigma \sqrt{2 \pi}} \frac{1}{x} e^{-(\ln x-\mu)^{2} /\left(2 \sigma^{2}\right)}
$$

(with $x \equiv N_{A}\langle\sigma v\rangle$ for short is the rate). This is equivalent to the assumption that $\ln (x)$ is Gaussian distributed with expectation value $\mu$ and variance $\sigma^{2}$ (both functions of temperature). The lognormal distribution allows to cope with large uncertainty factors $\left(\equiv e^{\sigma}\right)$ together with ensuring that the rates remain positive. If these parameters are tabulated as a function of the temperature, they can be used to perform subsequent Monte-Carlo nucleosynthesis calculations. The quantile of the distributions of the abundances obtained by such a method are reported in table VII for which we used 20000 Monte-Carlo points.

The variations of $Y_{\mathrm{P}}$ are nearly entirely due to the uncertainty on $\tau_{n}$ because it is almost completely controlled by weak rates which set the abundance of free neutrons before nucleosynthesis starts. This Monte-Carlo method allows to construct the probability $P\left(Y_{i} \mid \omega_{\mathrm{b}}\right)$ for each species, where $\omega_{\mathrm{b}} \equiv \Omega_{\mathrm{b}} h^{2}$. This probability reflects the uncertainty in all parameters affecting the reaction
TABLE VII Monte-Carlo estimation of light elements uncertainties due to nuclear rates, and $\tau_{n}$ (aka weak rates) uncertainties.

\begin{tabular}{llllllll}
\hline Quantile & $2.275 \%$ & $15.865 \%$ & $50 \%$ & $84.135 \%$ & $97.725 \%$ & mean & $\frac{\sigma}{\text { mean }}$ \\
\hline$Y_{\mathrm{P}}$ & 0.24676 & 0.24693 & 0.24709 & 0.24726 & 0.24742 & 0.24709 & $0.068 \%$ \\
$\mathrm{D} / \mathrm{H} \times 10^{5}$ & 2.386 & 2.423 & 2.460 & 2.496 & 2.532 & 2.459 & $1.49 \%$ \\
${ }^{3} \mathrm{He} / \mathrm{H} \times 10^{5}$ & 1.023 & 1.048 & 1.074 & 1.100 & 1.127 & 1.074 & $2.43 \%$ \\
${ }^{7} \mathrm{Li} / \mathrm{H} \times 10^{5}$ & 5.123 & 5.392 & 5.627 & 5.858 & 6.105 & 5.623 & $4.39 \%$ \\
${ }^{6} \mathrm{Li} / \mathrm{H} \times 10^{14}$ & 0.61 & 0.85 & 1.20 & 1.68 & 2.35 & 1.27 & $35 \%$ \\
$\mathrm{CNO} / \mathrm{H} \times 10^{15}$ & 0.14 & 0.52 & 1.02 & 3.07 & 65.6 & 15.3 & 13.4 \\
\hline
\end{tabular}

rates, independently of cosmology and it is used to plot the $\pm \sigma$ width of curved in Fig. 26 by computing the quantiles $\{0.15865,0.84135\}$.

For elements for which the uncertainty is small, it proves useful to approximate these probabilities by a normal distribution as

$$
P\left(Y_{i} \mid \omega_{\mathrm{b}}\right) \simeq \mathcal{N}\left[\bar{Y}_{i}\left(\omega_{\mathrm{b}}\right), \sigma_{i}^{\mathrm{th}}\left(\omega_{\mathrm{b}}\right)\right]\left(Y_{i}\right)
$$

where the normal distribution is noted

$$
\mathcal{N}[\mu, \sigma](x) \equiv \frac{1}{\sqrt{2 \pi} \sigma} \exp \left[-\frac{(x-\mu)^{2}}{2 \sigma^{2}}\right] .
$$

If we use the CMB to obtain a prior distribution on $\omega_{\mathrm{b}}$ that we assume to follow a normal distribution (the mean and standard deviation are reported in appendix $(\mathrm{D})$, then we can build the joint probability

$$
P\left(Y_{i}, \omega_{\mathrm{b}} \mid \mathrm{CMB}\right)=P\left(Y_{i} \mid \omega_{\mathrm{b}}\right) P\left(\omega_{\mathrm{b}} \mid \mathrm{CMB}\right),
$$

and marginalizing over $\omega_{\mathrm{b}}$ we get probabilities in predicted abundances

$$
P\left(Y_{i} \mid \mathrm{CMB}\right)=\int P\left(Y_{i}, \omega_{\mathrm{b}} \mid \mathrm{CMB}\right) \mathrm{d} \omega_{\mathrm{b}} .
$$

In practice we use also a Monte-Carlo method to obtain directly $P\left(Y_{i} \mid \mathrm{CMB}\right)$. We only need to vary $\omega_{\mathrm{b}}$, according to its normal distribution, in addition to varying the reaction rates. With this method we can predict the underlying abundances that are reported in table VIII. The predicted abundance for deuterium is noticeably lower than the one in Ade et al. (2016, Eq. 74) which uses PArthENoPE (Pisanti et al., 2008), and this has consequences when inferring the chemical potential of neutrinos (see $\S$ VI.C).

\section{F. Comparison with observations}

Except for ${ }^{4} \mathrm{He}$, and the weak rates, the predicted abundances, displayed in Table VIII, do not differ significantly from those of Coc et al. (2015). As can be seen in Table IV] very few reaction rates have been updated, and if so, either the rate changes are tiny or the reactions have, in any case, a negligible effect. On the contrary, there are significative differences with the results 
TABLE VIII Primordial abundances compared to observations.

\begin{tabular}{|c|c|c|c|c|c|}
\hline & Observations & $\begin{array}{c}(\mathrm{a}) \\
\tau_{n}=880.3(1.1) \mathrm{s}\end{array}$ & $\begin{array}{l}\text { This work (h) } \\
\tau_{n}=879.5(8) \mathrm{s}\end{array}$ & $\begin{array}{c}(\mathrm{f}) \\
\tau_{n}=880.3(1.1) \mathrm{s}\end{array}$ & $\begin{array}{l}\text { Planck } 2015(\mathrm{~g}) \\
\tau_{n}=880.3(1.1) \mathrm{s}\end{array}$ \\
\hline$Y_{\mathrm{P}}$ & $0.2449 \pm 0.0040(\mathrm{~b})$ & $0.2484 \pm 0.0002$ & $0.24709 \pm 0.00017$ & $0.24709 \pm 0.00025$ & $0.24667 \pm 0.00062$ \\
\hline $\mathrm{D} / \mathrm{H}\left(\times 10^{-5}\right)$ & $2.527 \pm 0.030(\mathrm{c})$ & $2.45 \pm 0.05$ & $2.459 \pm 0.036$ & $2.58 \pm 0.13$ & $2.614 \pm 0.13$ \\
\hline${ }^{3} \mathrm{He} / \mathrm{H}\left(\times 10^{-5}\right)$ & $<1.1 \pm 0.2(\mathrm{~d})$ & $1.07 \pm 0.03$ & $1.074 \pm 0.026$ & $1.0039 \pm 0.0090$ & \\
\hline${ }^{7} \mathrm{Li} / \mathrm{H}\left(\times 10^{-10}\right)$ & $1.58_{-0.28}^{+0.35}(\mathrm{e})$ & $5.61 \pm 0.26$ & $5.623 \pm 0.247$ & $4.68 \pm 0.67$ & \\
\hline
\end{tabular}

of Cyburt et al. (2016) which are mostly, and probably entirely, due to the different choice of reaction rates. Apparently, Cyburt et al. (2016) use for some of the most important reactions, $\mathrm{D}(\mathrm{p}, \gamma)^{3} \mathrm{He}, \mathrm{D}(\mathrm{d}, \mathrm{n})^{3} \mathrm{He}, \mathrm{D}(\mathrm{d}, \mathrm{p})^{3} \mathrm{H}$, and ${ }^{3} \mathrm{He}(\alpha, \gamma){ }^{7} \mathrm{Be}$, the rates from NACRE II $(\mathrm{Xu}$ et al. 2013), now superseded for these reactions by Coc et al. (2015); Gómez Iñesta et al. (2017); Iliadis et al. (2016). This is clearly the origin of the differences concerning D and ${ }^{7} \mathrm{Li}$. There is an even greater difference with the $\mathrm{D} / \mathrm{H}$ value reported by Planck (Ade et al., 2016; Di Valentino et al. 2014) using the PArthENoPE code (Pisanti et al., 2008). This is again, probably due to a different choice of reaction rates for deuterium destruction.

Since the results for deuterium and lithium are not significantly different from our earlier works (Coc et al. 2015, 2014) the comparison with observations is very similar. As explained in $\S$ I.A.3 we do not consider ${ }^{3} \mathrm{He}$ as a constraint.

\section{Helium}

Thanks to the re-evaluation of the corrections to the weak rates, we claim a precision of a few $10^{-4}$ on $Y_{\mathrm{P}}$ i.e. smaller than one unit on $Y_{\mathrm{P}}^{(4)}$ before taking into account the experimental uncertainty on the neutron lifetime and a few reaction rates ( $\S$ IV.C). We finally obtained $Y_{\mathrm{P}}=0.24705 \pm 0.00019$, fully consistent with the value $Y_{\mathrm{P}}=0.2449 \pm 0.0040$ deduced from Izotov et al. (2014) observations by Aver et al. (2015) (see Fig. 26), without the need for extra relativistic degrees of freedom as in (Izotov et al. 2014).

Since this work is focused on ${ }^{4} \mathrm{He}$ and is an improved continuation of previous works it is worthwhile tracking the evolution of our calculation of $Y_{\mathrm{P}}$. In our earliest works (e.g. Coc et al. (2004)), since the observational uncertainties on $Y_{\mathrm{P}}$ were large, we neglected some of the corrections discussed here because we were unable to calculate them (e.g. including six-fold integrals). The corrections that we took into account corresponds to line $\mathrm{RCa}$ line in Table $\mathrm{V}$ and our (Fortran) calculated corrections did amount to $\delta Y_{\mathrm{P}}^{(4)}=31.6 \sqrt{\mathrm{Coc}}$

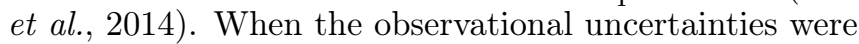
reduced, it become important to include the neglected corrections, by artificially increasing $Y_{\mathrm{P}}^{(4)}$ by 18 at the the very end of the calculation. It corresponded to the finite-nucleon mass correction $\left(\delta Y_{\mathrm{P}}^{(4)}=12\right.$ Lopez and Turner, 1999)), finite-temperature radiative correction $\left(\delta Y_{\mathrm{P}}^{(4)}=3\right)($ Lopez and Turner, 1999) $)$, QED plasma $\left(\delta Y_{\mathrm{P}}^{(4)}=1\right.$ (Lopez and Turner, 1999) $)$ and neutrino decoupling $\left(\delta Y_{\mathrm{P}}^{(4)}=2\right.$ (Mangano et al. $\left.\left.\mid 2005\right)\right)$, for a total of $\delta Y_{\mathrm{P}}^{(4)}=18$, that we could not easily directly re-calculate. Hence, before this final correction, the $Y_{\mathrm{P}}$ value from Coc et al. (2015) was 0.2466 (i.e. 0.2484 minus 0.0018 ) to be compared to 0.24572 (RCa in Table V). This difference of $\delta Y_{\mathrm{P}}^{(4)}=9$, which is in fact only $\delta Y_{\mathrm{P}}^{(4)}=7$ if we account for the different $\tau_{n}$ used, was reduced to $\delta Y_{\mathrm{P}}^{(4)} \simeq 0.5$ by improving the Fortran code (time steps, temperature grid,...) at the expense of execution time ${ }^{19}$.

\section{Deuterium}

Our present result differs by less than $3 \%$, and agrees within error bars with the latest value inferred from Cooke et al. (2018) observations. Since the theoretical value lies on the lower bound of the observational one, this leaves only little room for models of lithium (i.e. ${ }^{7} \mathrm{Be}$ ) destruction that byproduct extra deuterium (see below). Tiny differences with previous results (Coc et al., 2015) come in part from the corrections to the weak rates (see Table Vp, to the re-evaluation of the three nuclear reaction rates (Gómez Iñesta et al., 2017, Iliadis et al., 2016) that govern deuterium destruction and improvements in the numerical method. With the high precision reached by $\mathrm{D} / \mathrm{H}$ observations, these reaction rates $\left[\mathrm{D}(\mathrm{p}, \gamma)^{3} \mathrm{He}\right.$, $\mathrm{D}(\mathrm{d}, \mathrm{n})^{3} \mathrm{He}$ and $\left.\mathrm{D}(\mathrm{d}, \mathrm{p})^{3} \mathrm{H}\right]$ need to be known at the percent level! This demands accurate measurement at BBN energies where data are scarce and theoretical improvement to better constrain the energy dependence of the $S$-factors.

\footnotetext{
19 Details to appear elsewhere.
} 


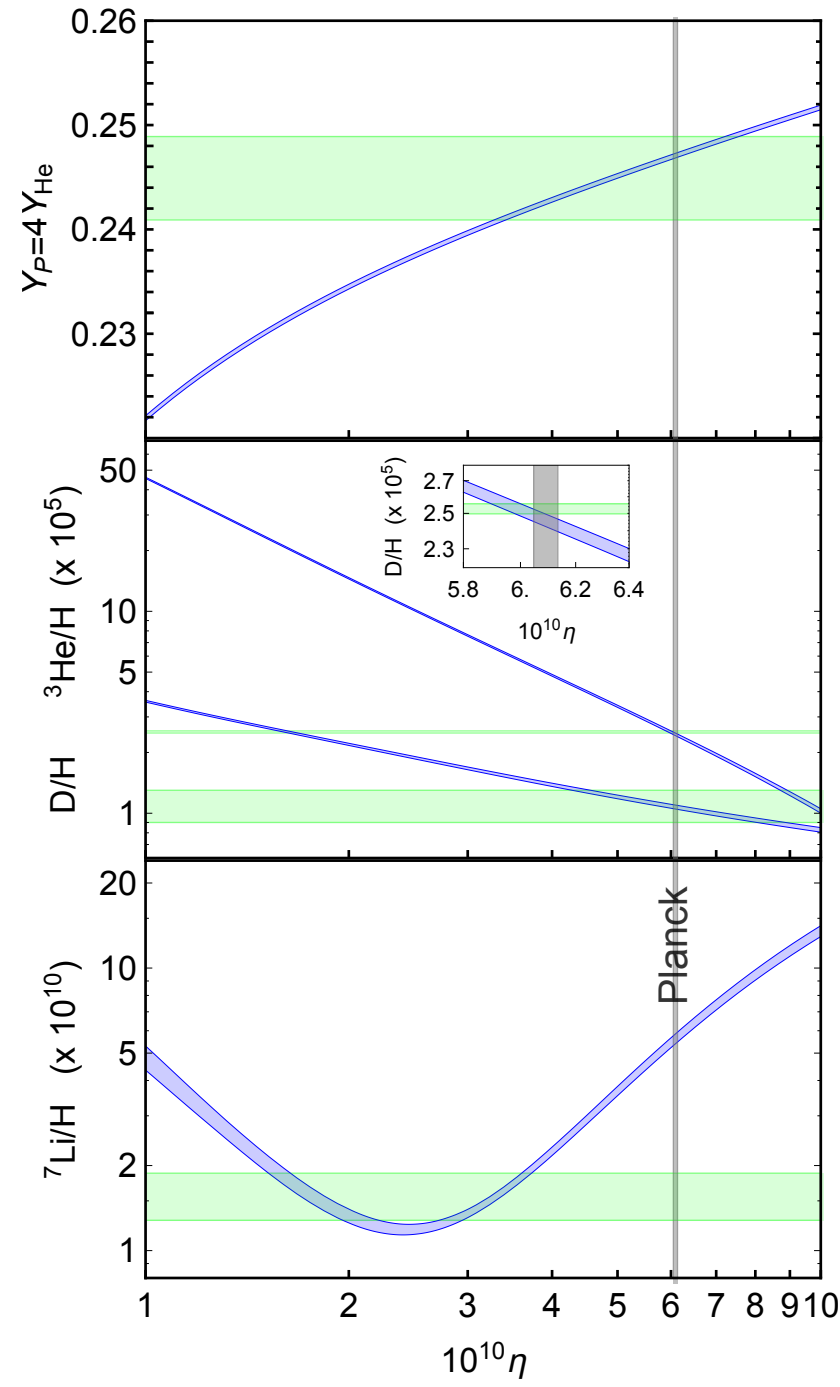

FIG. 26 Top : Dependence of $Y_{\mathrm{P}}=4 Y_{{ }^{4} \mathrm{He}}$ in $\eta$ and observational constraints. Middle: Dependence of deuterium (to curve) and ${ }^{3} \mathrm{He}$ (bottom curve) in $\eta$ with observational constraints. The ${ }^{3} \mathrm{H}$ has been added since it decays radioactively in ${ }^{3} \mathrm{He}$. Bottom : Dependence of ${ }^{7} \mathrm{Li}$ in $\eta$ with observational constraints. The ${ }^{7} \mathrm{Be}$ has been added since it decays radioactively in ${ }^{7} \mathrm{Li}$. In all these plots, the width of the curves represents the $\pm \sigma$ uncertainty from nuclear rates and neutron lifetime.

\section{Lithium}

There remains a factor of 3.6 between the predicted and observed $\mathrm{Li} / \mathrm{H}$ values. This discrepancy has not yet found a fully satisfactory solution. On the contrary, the problem had worsened because of an updated reaction rate (Cyburt and Davids, 2008; Cyburt et al. 2008) and because many solutions have been ruled out by the improved precision on $\mathrm{D} / \mathrm{H}$ observations Cooke et al. 2018). We present below the kinds of solutions that have been considered so far.
- There is no nuclear solution to the lithium problem. Extensive sensitivity studies (Coc et al., 2012, 2004) have not identified reactions, beyond those already known, that could have a strong impact on lithium nucleosynthesis. The most promising was ${ }^{7} \mathrm{Be}(\mathrm{d}, \mathrm{p}) 2 \alpha$ (Coc et al. 2004 , Cyburt and Pospelov, 2012): an increase of its rate by a factor of $\approx 100$ would have solved the problem. However, measurements of its average cross section (Angulo et al. 2005 ) or properties of candidate resonances (Kirsebom and Davids, 2011, O'Malley et al., 2011; Scholl et al. (2011) ruled out this possibility. More generally, other destruction channels have recently been proposed (Chakraborty et al. 2011$):{ }^{7} \mathrm{Be}+\mathrm{n}, \mathrm{p}, \mathrm{d}$, $\mathrm{t},{ }^{3} \mathrm{He}$ and ${ }^{4} \mathrm{He}$. In particular, the existence of a relatively narrow state around $15 \mathrm{MeV}$ in the compound nucleus ${ }^{10} \mathrm{C}$ formed by ${ }^{7} \mathrm{Be}+{ }^{3} \mathrm{He}$ or the existence of a state close to $8 \mathrm{MeV}$ in the compound nucleus ${ }^{11} \mathrm{C}$ formed by ${ }^{7} \mathrm{Be}+{ }^{4} \mathrm{He}$ could help reduce the ${ }^{7}$ Be production. However, a recent search (Hammache et al. , 2013) for missing levels in the relevant excitation energy regions of ${ }^{10} \mathrm{C}$ and ${ }^{11} \mathrm{C}$, via the reactions ${ }^{10} \mathrm{~B}\left({ }^{3} \mathrm{He}, \mathrm{t}\right){ }^{10} \mathrm{C}$ and ${ }^{11} \mathrm{~B}\left({ }^{3} \mathrm{He}, \mathrm{t}\right){ }^{11} \mathrm{C}$, respectively, did not find any new level, whose corresponding resonances, in any case, would have too low strengths (Broggini et al., 2012 ) because of the Coulomb barrier. It seems now that all extra ${ }^{7} \mathrm{Be}$ destructing reactions have been considered and found inefficient.

- The effect of electron screening or modification of decay lifetime is negligible. For reactions of interest to BBN, screening affects the laboratory cross sections at too low energies [e.g. $\lesssim 20 \mathrm{keV}$ for $\mathrm{D}(\mathrm{d}, \mathrm{p})^{3} \mathrm{H}$ (Greife et al., 1995) or ${ }^{3} \mathrm{He}(\mathrm{d}, \mathrm{p})^{4} \mathrm{He}$ (Aliotta et al., 2001)] to affect measurement at $\mathrm{BBN}$ energies $\approx 100 \mathrm{keV}]$, on the one hand. On the other hand, the effect of screening during BBN is completely negligible (Famiano et al., 2016, Wang et al., 2011). It is well known that the lifetime of ${ }^{7} \mathrm{Be}$ that decays by electron capture depends on the probability of presence of an electron (from an atomic $s$ orbital or from a plasma) inside the nucleus; for instance it is increased to $\sim 100$ days at the center of the Sun (Adelberger et al. 2011). To have an impact on lithium prediction, the ${ }^{7} \mathrm{Be}$ lifetime must be reduced to a value of $\sim 10^{3} \mathrm{~s}$. However, because of the Boltzmann suppression factor, at $T<0.5 \mathrm{GK}$, when ${ }^{7} \mathrm{Be}$ is present, the electron density becomes smaller than in the Sun so that one can expect an even longer lifetime. This can be confirmed if one extrapolates the results of $\mathrm{Si}-$ monucci et al. (2013, Fig. 1), to $T \lesssim 500 \times 10^{6} \mathrm{~K}$ and $\rho \lesssim 10^{-5} \mathrm{~g} / \mathrm{cm}^{3}$.

- Many exotic solutions to the lithium problem have been investigated (e.g. Yamazaki et al. (2014)), but most rely on extra neutron sources to boost ${ }^{7} \mathrm{Be}$ destruction through the ${ }^{7} \mathrm{Be}(\mathrm{n}, \mathrm{p}){ }^{7} \mathrm{Li}(\mathrm{p}, \alpha){ }^{4} \mathrm{He}$ 
channel. However, these extra neutrons, inevitably, also boost the $\mathrm{D}$ and ${ }^{3} \mathrm{H}$ production through the ${ }^{1} \mathrm{H}(\mathrm{n}, \gamma){ }^{2} \mathrm{H}$ and ${ }^{3} \mathrm{He}(\mathrm{n}, \mathrm{p}){ }^{3} \mathrm{H}$ channels, respectively (Coc et al., 2015; Kusakabe et al., 2014). This is shown in Figs. 1 in Olive et al. (2012), 4 in Kusakabe et al. (2014), and 14 in Coc et al. (2015) that display results of various types of models that succeed in solving the lithium problem, but at the expense of deuterium overproduction to levels now excluded by observations (Cooke et al. 2014, 2016. 2018). Very few solutions, even beyond the Standard Model, that do not suffer from this drawback are left, e.g. Goudelis et al. (2016).

- Stellar physics solutions require a uniform reduction of surface lithium over a wide range of effective temperature and metallicity. Some amount of surface lithium destruction is unavoidable, because of atomic diffusion that transport lithium down to deeper and hotter layers where it is destroyed by a factor of 1.5 to 2 (Michaud et al. 1984). The difficulty comes from the small thickness of the lithium plateau over a wide range of metallicity and temperature and the absence of stars between the plateau and the BBN prediction (Fig. 2). This could possibly be circumvented if an additional mixing process is included in the outer layers of these stars (Richard et al. , 2005). This is supported by the comparison between lithium observations in the metal-poor globular cluster NGC 6397 with stellar depletion models (Korn et al., 2006). Another recent proposition relies on full lithium destruction but followed by a self-regulated reenrichment of lithium by late time accretion from the interstellar gas ( $\mathrm{Fu}$ et al., 2015$)$. In addition, the Spite plateau does not exist anymore at the metallicity below $[\mathrm{Fe} / \mathrm{H}]=-3$, and is replaced by an increased spread of abundances, below the plateau value (Sbordone et al. 2010). This "meltdown" of the Spite plateau is not understood yet. All this suggests that lithium observations cannot be used anymore to constrain BBN models.

- There is no ${ }^{6} \mathrm{Li}$ problem anymore. A few years ago, observations (Asplund et al. 2006) of ${ }^{6} \mathrm{Li}$ in a few metal poor stars had suggested the presence of a plateau, at typically ${ }^{6} \mathrm{Li} / \mathrm{H} \approx 10^{-11}$, orders of magnitude higher than the BBN predictions of ${ }^{6} \mathrm{Li} / \mathrm{H} \approx 1.3 \times 10^{-14}$ (Hammache et al. 2010 ). However, later, the observational ${ }^{6} \mathrm{Li}$ plateau has been questioned due to line asymmetries which were neglected in previous abundance analyses. Hence, there is no remaining evidence for a plateau at very low metallicity (Lind et al., 2013 ) that can be used to derive a primordial ${ }^{6} \mathrm{Li}$ abundance.

\section{Other elements}

Leaving aside the ${ }^{9} \mathrm{Be},{ }^{10} \mathrm{~B}$ and ${ }^{11} \mathrm{~B}$ isotopes for which no primordial abundance can be inferred from observations (Coc et al. . 2014), it is worth mentioning the CNO abundance. Here, we call CNO all isotopes with masses larger than $12\left({ }^{11} \mathrm{C}\right.$ mostly decays to $\left.{ }^{11} \mathrm{~B}\right)$. Even though, there are no primordial $\mathrm{CNO}$ abundance either, it is of peculiar interest since it may affect the evolution of the first stars (Population III) within the first structures of the Universe. Hydrogen burning in the first generation of stars proceeds through the slow pp-chains until enough carbon is produced (through the triple-alpha reaction) to activate the CNO cycle. The minimum value of the initial CNO mass fraction that would affect Population III stellar evolution is estimated to be $10^{-11}$ (Cassisi and Castellani. 1993) or even as low as $10^{-13}$ (in number of atoms relative to hydrogen, $\mathrm{CNO} / \mathrm{H}$ ) for the less massive stars (Ekström et al. 2008). Table VII shows that the median abundance (0.5 quantile) is in agreement with previous works (Coc et al., 2012, Iocco et al., 2007), if taking into account that the distribution is not Gaussian (Coc et al. 2014, Coc and Vangioni, 2014). As a result, the 0.97725 quantile corresponds to $\mathrm{CNO} / \mathrm{H} \approx 0.7 \times 10^{-13}$, close to the limit to have an impact on some first stars. As this is explained in Coc et al. (2014), this value, much larger than the median comes from the simultaneous variations, during the Monte Carlo, of a few reaction rates around ${ }^{10} \mathrm{Be}$. Since very few or no experimental data are available for these reactions, the actual rates may differ by a few orders of magnitudes, from the TALYS Goriely et al. 2008) theoretical predictions. Experimental efforts are needed to confirm or infirm this possibility.

\section{COSMOLOGY WITH BBN}

\section{A. Cosmological perturbations}

Cosmological fluctuations are of order $10^{-5}$, and whatever their effect, we have neglected them given our precision goal. However it is easy to estimate the effect of adiabatic perturbations seeded by inflation. Indeed, since the Hubble radius is extremely small during $\mathrm{BBN}$, most modes can be considered as super-Hubble modes, that is longer than the Hubble radius. In that case their effect can be described by a simple coordinate transformation applied on a homogeneous cosmology (Creminelli et al. 2011: Mirbabayi and Zaldarriaga, 2015; Weinberg, 2003).

Let us consider a perturbed metric (expressed in conformal time defined by $\mathrm{d} t=a \mathrm{~d} \eta$ )

$$
\mathrm{d} s^{2}=a^{2}(\eta)\left(-(1+2 \Phi) \mathrm{d} \eta^{2}+(1-2 \Psi) \delta_{i j} \mathrm{~d} x^{i} \mathrm{~d} x^{j}\right) .
$$

It can be put in the homogeneous form using a coordinate transformation. Or conversely the dynamics of a spacetime perturbed by long modes can be deduced from the homogeneous dynamics thanks to a coordinate transformation. Not all such transformations lead to a physical 
long mode dynamics, and one must ensure some conditions. In the case where the Universe dynamics is dominated by radiation, as is the case for $\mathrm{BBN}$, the relevant coordinate transformation is (Creminelli et al., 2011)

$$
\tilde{\eta}=\eta\left(1-\frac{\zeta}{3}\right), \quad \tilde{x}^{i}=x^{i}(1+\zeta)
$$

where $\zeta$ is the comoving curvature perturbation.

It follows using Eq. 10 that the number density in the perturbed universe $\tilde{n}_{i}$ is related to the one of a homogeneous cosmology $n_{i}$ thanks to

$$
\tilde{n}_{i}=n_{i}-\frac{\eta \zeta}{3} \partial_{\eta} n_{i}=(1+\zeta) n_{i}-\frac{\eta \zeta}{3} a \mathcal{J}_{i},
$$

where we used that for radiation domination $a \propto \eta$ and $\partial_{\eta} a / a=1 / \eta$. At the end of $\mathrm{BBN}$, when all reactions are inefficient, all net creation rates $\mathcal{J}_{i}$ vanish and we find that all number densities are simply rescaled by $1+\zeta$. Note that this could have been anticipated because for super-Hubble modes, $\zeta$ is equal to density perturbations on isocurvature surfaces (Vernizzi, 2005). Since abundances are ratios of number densities, they remain unchanged by the long mode perturbation. Hence, provided we can neglect the effect of modes which are smaller than the Hubble radius during BBN, standard adiabatic cosmological perturbations have no effect on BBN predictions. Intuitively, an adiabatic perturbation enhances baryon and photons number densities in the same proportions, leaving $\eta$ constant. Since this analysis is based on long modes only, one cannot infer the consequences of fluctuations on scales smaller than the Hubble radius during $\mathrm{BBN}$ from the homogeneous cosmology results. For these small scales, only a complete treatment of all perturbed equations dictating the evolution of species can lead to a meaningful result.

Finally, it is worth noting that long modes of entropy perturbation, that is perturbations which do not modify the total energy density (and for which $\zeta=0$ ) but which modify the ratios $\rho_{\mathrm{b}} / \rho_{\gamma}$ have an effect on abundances. Since during BBN the total energy density is dominated by radiation, and long mode entropy perturbation can be rephrased as a perturbation in $\rho_{\mathrm{b}}$ alone, that is a perturbation of $\eta$. As a consequence, its effect is directly evaluated by the sensitivity of BBN final abundances on $\eta$.

\section{B. Measurement of baryon abundance from BBN}

The observed abundances are related to the underlying one by an assumed normal distribution, that is for each isotope observed, the likelihood is

$$
P\left(Y_{i}^{\mathrm{obs}} \mid Y_{i}\right)=\mathcal{N}\left[Y_{i}, \sigma_{i}^{\mathrm{obs}}\right]\left(Y_{i}^{\mathrm{obs}}\right) .
$$

The observed values and their standard deviations are reported in $\S$ I.A. Then we can consider

$$
P\left(Y_{i}^{\mathrm{obs}} \mid \omega_{\mathrm{b}}\right) \equiv \int \mathrm{d} Y_{i} P\left(Y_{i}^{\mathrm{obs}} \mid Y_{i}\right) P\left(Y_{i} \mid \omega_{\mathrm{b}}\right),
$$

and we deduce using Eqs. (148) and 155 that it follows approximately a normal distribution

$$
P\left(Y_{i}^{\mathrm{obs}} \mid \omega_{\mathrm{b}}\right) \simeq \mathcal{N}\left[\bar{Y}_{i}\left(\omega_{\mathrm{b}}\right), \Sigma_{i}\left(\omega_{\mathrm{b}}\right)\right]\left(Y_{i}^{\mathrm{obs}}\right)
$$

with $\Sigma_{i}^{2}\left(\omega_{\mathrm{b}}\right) \equiv\left(\sigma_{i}^{\mathrm{obs}}\right)^{2}+\left[\sigma_{i}^{\text {th }}\left(\omega_{\mathrm{b}}\right)\right]^{2}$. In practice we do not use the approximation (148) and use instead the full result of our Monte-Carlo method to estimate the distribution of abundances due to reaction rates uncertainties. However, the results obtained are extremely similar given that 148 is a very good approximation.

If we then use an uniform prior on $\omega_{\mathrm{b}}$, that is if we do not use our knowledge from CMB observations, the posterior distribution for $\omega_{\mathrm{b}}$ is immediately given from $P\left(\omega_{\mathrm{b}} \mid Y_{i}^{\text {obs }}\right) \propto P\left(Y_{i}^{\mathrm{obs}} \mid \omega_{\mathrm{b}}\right)$. Otherwise we use the CMB prior to build the posterior distribution for $\omega_{\mathrm{b}}$ as

$$
\begin{aligned}
P\left(\omega_{\mathrm{b}} \mid Y_{i}^{\mathrm{obs}}, \mathrm{CMB}\right) & \propto P\left(Y_{i}^{\mathrm{obs}}, \omega_{\mathrm{b}} \mid \mathrm{CMB}\right) \\
& =P\left(Y_{i}^{\mathrm{obs}} \mid \omega_{\mathrm{b}}\right) P\left(\omega_{\mathrm{b}} \mid \mathrm{CMB}\right)
\end{aligned}
$$

When including all observed abundances, we use that the total probability is the product of individual ones. In practice, only ${ }^{4} \mathrm{He}$ and ${ }^{2} \mathrm{H}$ are determined with enough precise experimental precision to be taken into account.

The CMB prior distribution and the posterior distribution from the observations of $\mathrm{BBN}$ are plotted in Fig. 27. The posterior bounds from $\mathrm{BBN}$ and $\mathrm{CMB}$ are $\Omega_{\mathrm{b}} h^{2}=0.02215 \pm 0.00014$, whereas from BBN alone on gets $\Omega_{\mathrm{b}} h^{2}=0.02190 \pm 0.00025$. Note that if we had used a code which predicts $\mathrm{D} / \mathrm{H} \simeq 2.61 \times 10^{-5}$ instead of $\mathrm{D} / \mathrm{H} \simeq 2.46 \times 10^{-5}$ for the Planck parameters, we would have obtained the constraint $\Omega_{\mathrm{b}} h^{2}=0.02271 \pm 0.00025$ from $\mathrm{BBN}$ alone, having exactly the same central value than found in Consiglio et al. (2017), using PArthENoPE.

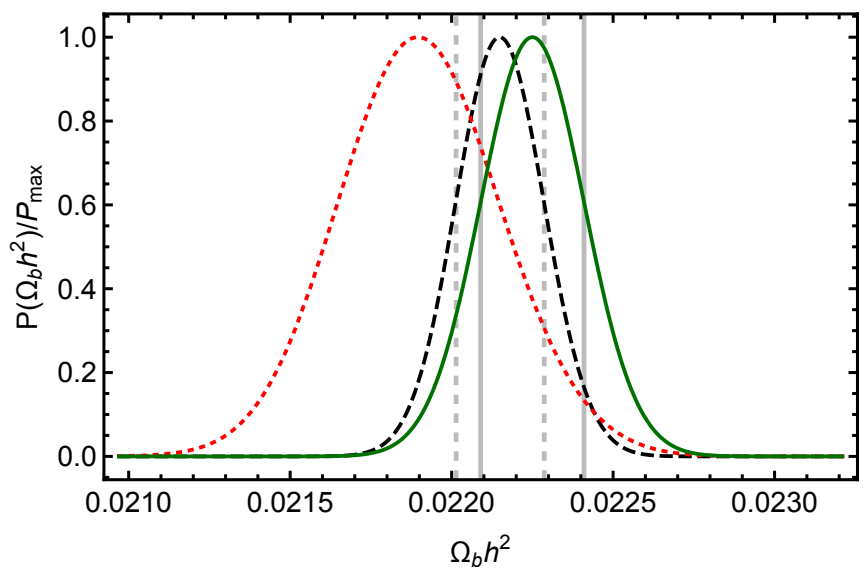

FIG. $27 P\left(\Omega_{\mathrm{b}} h^{2}\right)$ normalized to a unit maximum. Green continuous line: $\mathrm{CMB}$ prior distribution. Black dashed line $\mathrm{BBN}$ posterior distribution $(\mathrm{BBN}+\mathrm{CMB})$. Red dotted line : baryon abundance distribution determined only from BBN. The vertical gray lines are the $\pm \sigma \mathrm{CMB}$ (continuous) and $\mathrm{CMB}+\mathrm{BBN}$ (dashed) bounds. 


\section{Neutrino chemical potential from BBN}

If there is a neutrino asymmetry, that is a nonvanishing neutrino chemical potential, then for each flavor we have necessarily

$$
\mu_{\nu}+\mu_{\bar{\nu}}=0
$$

because of processes like $\nu+\bar{\nu} \leftrightarrow e^{-}+e^{+} \leftrightarrow 2 \gamma$ and photons have a vanishing chemical potential. Furthermore cosmological expansion affects only the particle momenta and $\xi_{\nu} \equiv \mu_{\nu} / T_{\nu}$ is frozen at its initial value, whose consequences on BBN can be investigated (Iocco et al., 2009 Serpico and Raffelt, 2005, Simha and Steigman, 2008).

The neutrino asymmetry (defined for each neutrino flavor) is defined by the excess of neutrinos over antineutrinos as

$$
\begin{aligned}
\eta_{\nu} \equiv \frac{n_{\nu}-n_{\bar{\nu}}}{n_{\gamma}} & =\frac{I_{+}^{(1,1)}\left(0, \xi_{\nu}\right)-I_{+}^{(1,1)}\left(0,-\xi_{\nu}\right)}{2 I_{-}^{(1,1)}} \\
& \simeq \frac{1}{12 \zeta(3)}\left(\frac{T_{\nu}}{T}\right)^{3}\left(\pi^{2} \xi_{\nu}+\xi_{\nu}^{3}\right)
\end{aligned}
$$

The neutrino oscillations imply that the various flavors must reach an equilibrium for which the chemical potentials are equal (Dolgov et al. 2002, Wong, 2002). Assuming accordingly that the asymmetry is the same for all flavors, the first modification of the neutrino asymmetry is an excess of energy densities stored in neutrinos which can be absorbed by a redefinition of the number of neutrino generations $N_{\nu}$ as

$$
\begin{aligned}
\tilde{N}_{\nu} & =N_{\nu}\left(1+\frac{I_{+}^{(2,1)}\left(0, \xi_{\nu}\right)+I_{+}^{(2,1)}\left(0,-\xi_{\nu}\right)}{2 I_{+}^{(2,1)}}\right) \\
& \simeq N_{\nu}\left(1+\frac{30 \xi_{\nu}^{2}}{7 \pi^{2}}+\frac{15 \xi_{\nu}^{4}}{7 \pi^{4}}\right) .
\end{aligned}
$$

This effect is very small for small values of $\xi_{\nu}$ since it is at least quadratic in $\xi_{\nu}$.

There is a second modification for the weak-interaction rates in which one must use instead of Eq. 75

$$
g_{\nu}(E) \equiv \begin{cases}g_{\nu}^{+}(E) \equiv \frac{1}{\mathrm{e}^{\left(E / T_{\nu}-\xi_{\nu}\right)}+1} & n \rightarrow p \text { rates } \\ g_{\nu}^{-}(E) \equiv \frac{1}{\mathrm{e}^{\left(E / T_{\nu}+\xi_{\nu}\right)}+1} & p \rightarrow n \text { rates }\end{cases}
$$

For instance in the definition 790 of $\chi_{ \pm}$, one must use $g_{\nu}^{ \pm}$instead of $g_{\nu}$. To show this we used that the Pauliblocking factor of the antineutrino (resp. neutrino) is still related to the distribution of the neutrino (resp. antineutrino) according to 72 thanks to the property (158). This modification is the most important one and modifies the thermodynamical equilibrium ratio between neutrons and protons (A15) because from Eq. A14 we get $\mu_{n}-\mu_{p}=-\mu_{\nu}$. We get that at thermodynamical equilibrium

$$
\frac{n_{n}}{n_{p}}=\left.\frac{n_{n}}{n_{p}}\right|_{\xi_{\nu}=0} \times \mathrm{e}^{-\xi_{\nu}}
$$

and one expects a modification of the freeze-out abundance (for small $\xi_{\nu}$ ) of order

$$
\frac{\Delta Y_{n}^{F}}{Y_{n}^{F}} \simeq \frac{\Delta Y_{\mathrm{P}}}{Y_{\mathrm{P}}} \simeq-\left(1-Y_{n}^{F}\right) \xi_{\nu} .
$$

Finally there is a third modification when one considers the effect of incomplete neutrino decoupling (Grohs et al., 2017) but this is negligible since the effect without the asymmetry is already very small. For small $\xi_{\nu}$ we find the abundance modifications

$$
\begin{aligned}
\frac{\Delta Y_{\mathrm{P}}}{Y_{\mathrm{P}}} & \simeq-0.96 \xi_{\nu} \\
\frac{\Delta \mathrm{D} / \mathrm{H}}{\mathrm{D} / \mathrm{H}} & \simeq-0.53 \xi_{\nu} \\
\frac{\Delta^{3} \mathrm{He} / \mathrm{H}}{{ }^{3} \mathrm{He} / \mathrm{H}} & \simeq-0.18 \xi_{\nu} \\
\frac{\Delta^{7} \mathrm{Li} / \mathrm{H}}{{ }^{7} \mathrm{Li} / \mathrm{H}} & \simeq-0.62 \xi_{\nu} .
\end{aligned}
$$

The modification of $Y_{\mathrm{P}}$ is in very good agreement with the estimation 163 since $\left(1-Y_{n}^{F}\right) \simeq 0.92$. Since BBN has no free parameter when assuming non-degenerate neutrinos $\left(\xi_{\nu}=0\right)$, the observational constraints on $Y_{\mathrm{P}}$ and $\mathrm{D} / \mathrm{H}$ can be used to obtain bounds on the degeneracy parameter $\xi_{\nu}$ if we consider instead that it is unknown.

We repeat the analysis of $\S$ VI.B, using the pair of cosmological parameters $\left(\omega_{\mathrm{b}}, \xi_{\nu}\right)$ instead of $\omega_{\mathrm{b}}$ alone. However, we still assume that the CMB prior determines only $\omega_{\mathrm{b}}$. Again, for each species the uncertainty from nuclear rates $\sigma_{i}^{\text {th }}\left(\omega_{\mathrm{b}}, \xi_{\nu}\right)$ is obtained from a Monte-Carlo method. The posterior distribution for $\left(\omega_{\mathrm{b}}, \xi_{\nu}\right)$ from BBN combined with $\mathrm{CMB}$ is plotted in Fig. 28. Once marginalized over baryon abundance we get

$$
\xi_{\nu}=0.001 \pm 0.016
$$

which is a much tighter constraint than the constraints from CMB alone (Oldengott and Schwarz, 2017, $\left.\xi_{\nu}=-0.002_{-0.060}^{+0.053}\right)$ or than earlier BBN constraints such as Simha and Steigman $\left(2008, \xi_{\nu}=0.037 \pm 0.026\right)$, thanks to the recent improvement on both deuterium and ${ }^{4} \mathrm{He}$ abundance measurements.

Even though the bounds 165 are tighter, they are also surprisingly still highly compatible with a vanishing neutrino chemical potential. Since we find $\left|\xi_{\nu}\right|<$ $0.016(68 \% \mathrm{CL})$, it corresponds to $\left(\tilde{N}_{\nu}-N_{\nu}\right) / N_{\nu}<$ $0.011 \%$ and $\eta_{\nu}<4.0 \times 10^{-3}$.

Note that if we had used Parthenope (Pisanti et al. 2008), as is the case in Ade et al. (2016), which predicts $\mathrm{D} / \mathrm{H} \simeq 2.61 \times 10^{-5}$ rather than $\mathrm{D} / \mathrm{H} \simeq 2.46 \times 10^{-5}$ for Planck parameters, we would have found rather $\xi_{\nu}=$ $0.021 \pm 0.016$ less compatible with a vanishing neutrino chemical potential. Indeed, in our case the observed value for $Y_{\mathrm{P}}($ resp. $\mathrm{D} / \mathrm{H})$ is lower (resp. higher) than the averaged value inferred from the $\mathrm{CMB}$ measured baryon abundance, and given the linearized dependences 164 
which have the same sign, this results in a compensation and a central value very close to zero. However if the prediction for deuterium from a BBN code is also above its observed value, then both $Y_{\mathrm{P}}$ and $\mathrm{D} / \mathrm{H}$ tend to favor a positive $\xi_{\nu}$.

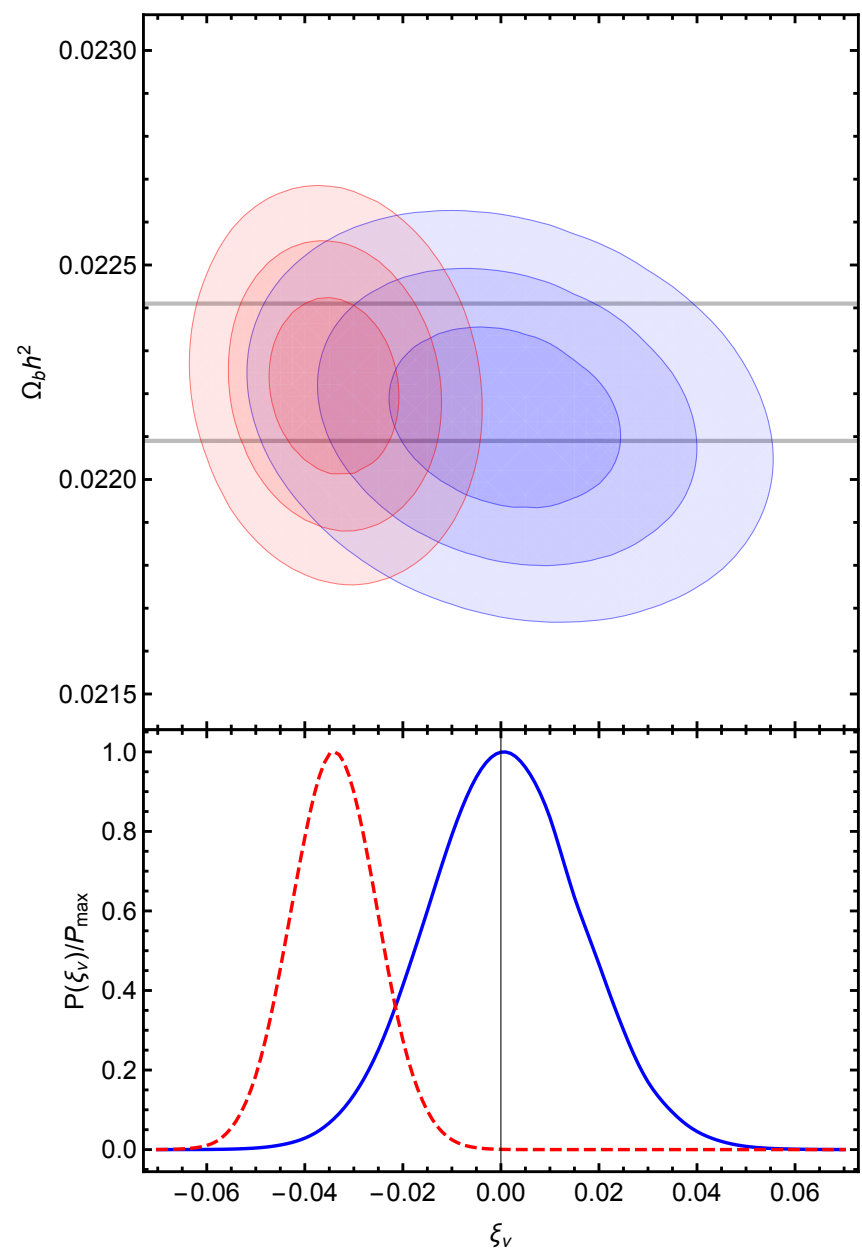

FIG. 28 Top : $P\left(\Omega_{\mathrm{b}} h^{2}, \xi_{\nu}\right)$ with $68.27 \%, 95.45 \%$ and $99.73 \%$ contours. Blue : using the ${ }^{4} \mathrm{He}$ bounds (4). Red : using the bounds $Y_{\mathrm{P}}=0.2551 \pm 0.0022$ of Izotov et al. (2014). The gray horizontal bars are the $\pm \sigma \mathrm{CMB}$ constraints on baryon abundance. Bottom : marginalized distribution for $\xi_{\nu}$. Continuous line uses (4) whereas the dashed line uses the bounds $Y_{\mathrm{P}}=0.2551 \pm 0.0022$ of Izotov et al. (2014).

\section{Number of neutrinos}

We repeat the analysis of $\S$ VI.B but for the pairs of cosmological parameters $\left(\Omega_{\mathrm{b}} h^{2}, N_{\nu}\right)$ instead of the baryon abundance alone. We use the CMB prior $P\left(\Omega_{\mathrm{b}} h^{2}, N_{\nu} \mid \mathrm{CMB}\right)$ obtained from the MonteCarlo Markov chains base_nnu_plikHM_TTTEEE_lowTEB of Ade et al. (2016) (TT+ $\mathrm{TE}+\mathrm{EE}+\mathrm{low} \mathrm{P}$ analysis). The constraints from CMB are given directly in terms of $N_{\text {eff }}$ (see $\S$ II.G) since they are obtained as the modelindependent gravitational contribution of relativistic de- grees of freedom. However, during BBN the effective number of neutrinos evolves as can be seen in Fig. 10, and this evolution is model-dependent. We assume that if there are extra relativistic degrees of freedom, they do not share any of the energy which is brought to neutrinos by incomplete decoupling. Hence for these extra neutrinos, or what is described phenomenologically as extra neutrinos, $z_{\nu}=1$ throughout BBN. Given these assumptions Eq. 66 which was derived for $N_{\nu}=3$ becomes

$$
N_{\text {eff }}=3\left(\frac{z_{\nu} z^{\text {stand }}}{z}\right)^{4}+\left(N_{\nu}-3\right)\left(\frac{z^{\text {stand }}}{z}\right)^{4}
$$

from which we deduce in particular that $\mathrm{d} N_{\text {eff }} / \mathrm{d} N_{\nu}=$ $\left(z^{\text {stand }} / z\right)^{4} \simeq 1.0090$. In fact, this relation is unchanged as long as the energy exchange between the plasma and the neutrino sector remains identical to Eq. (60), that is if the function $\mathcal{N}(T)$ is unchanged. More generally the effective number of neutrinos is insensitive to any type of neutrino spectral distortions which leaves the total neutrino energy density unchanged. Hence, as long as the energy transfer with the plasma is the same, we can also assume that the new neutrino degrees of freedom share a part of the energy transfer during the incomplete decoupling phase, and still use Eq. (166). This relation allows to convert the distribution $P\left(\Omega_{\mathrm{b}} h^{2}, N_{\text {eff }} \mid \mathrm{CMB}\right)$ into $P\left(\Omega_{\mathrm{b}} h^{2}, N_{\nu} \mid \mathrm{CMB}\right)$. This approach is different from the one chosen by Cyburt et al. (2016) where Eq. (66) is assumed to hold even for $N_{\nu} \neq 3$. However, given the rather large observational bounds on $N_{\nu}$ obtained, and the smallness of $z_{\nu}^{4}$, this difference in the conversion of neutrino numbers is not crucial.

The posteriors obtained are depicted in Fig. 29. They are to be compared with the plots of Cyburt et al. (2016, Fig. 10). Furthermore, it presents a significant improvement compared to Cooke et al. (2018, Fig. 7) thanks to the inclusion of the measured $Y_{\mathrm{P}}$ in the BBN constraint. We used the full results of the chains for the CMB prior, but it can be very well represented by a two-dimensional Gaussian distribution with $\Omega_{\mathrm{b}} h^{2}=0.022197 \pm 0.000245$, $N_{\nu}=2.945 \pm 0.203$ and a rather strong correlation coefficient $r=0.7699$, since this approximation affects only marginally the posteriors obtained. Once marginalized over baryon abundance, the constraints on the numbers of neutrinos are

$$
N_{\nu}= \begin{cases}2.95 \pm 0.20 & \mathrm{CMB} \\ 2.88 \pm 0.27 & \mathrm{BBN} \\ 3.01 \pm 0.15 & \mathrm{BBN}+\mathrm{CMB}\end{cases}
$$

As for the marginalized baryon abundances we get

$$
100 \times \Omega_{\mathrm{b}} h^{2}= \begin{cases}2.220 \pm 0.025 & \mathrm{CMB} \\ 2.168 \pm 0.055 & \mathrm{BBN} \\ 2.216 \pm 0.022 & \mathrm{BBN}+\mathrm{CMB}\end{cases}
$$

Note that if we had used a code which predicts $\mathrm{D} / \mathrm{H} \simeq$ $2.61 \times 10^{-5}$ instead of $\mathrm{D} / \mathrm{H} \simeq 2.46 \times 10^{-5}$ for the Planck 


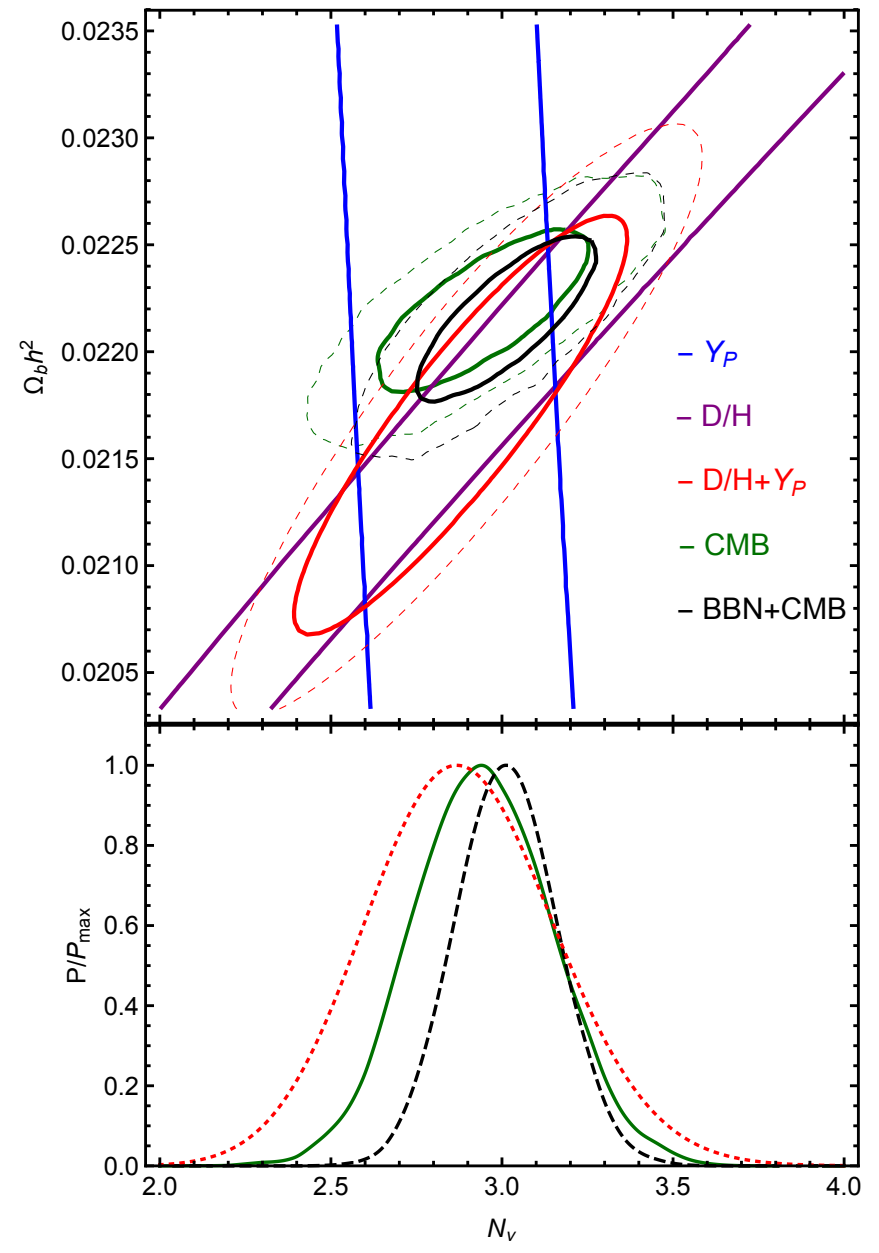

FIG. 29 Top : $P\left(\Omega_{\mathrm{b}} h^{2}, N_{\nu}\right)$. with $68.27 \%$ and $95.45 \%$ contours for different combinations of data. Bottom : $P\left(N_{\nu}\right)$ from marginalization. Continuous green is from CMB only, dotted red from BBN only, and dashed black is the combination of BBN and CMB. Note that the average value of $N_{\nu}$ for the combination of $\mathrm{BBN}$ and $\mathrm{CMB}$ is not between the corresponding averages obtained from $\mathrm{CMB}$ and $\mathrm{BBN}$ considered separately. There is no contradiction since the nearly elliptic preferred regions in the $\left(\Omega_{\mathrm{b}} h^{2}, N_{\nu}\right)$ space for BBN and CMB taken separately overlap away from the line defined by their respective average points.

parameters, we would have obtained $N_{\nu}=2.84 \pm 0.27$ from BBN alone, corresponding to $N_{\text {eff }}=2.88 \pm 0.27$, whose central value is similar to the one obtained in Consiglio et al. (2017, Eq. 26). Since we use consistently the CMB prior $P\left(\Omega_{\mathrm{b}} h^{2}, N_{\nu} \mid \mathrm{CMB}\right)$ when obtaining constraints from $\mathrm{BBN}$ and $\mathrm{CMB}$, we cannot compare our results with those of Consiglio et al. (2017) in that case, since they use only a CMB prior on $\Omega_{\mathrm{b}} h^{2}$.

\section{Conclusion}

It is widely acknowledged that Cosmology has entered in the "precision era"; this should also apply to big bang nucleosynthesis. For both $\mathrm{D}$ and ${ }^{4} \mathrm{He}$ isotopes the precision on the abundances deduced from observations have reached the percent level. The precision on primordial D abundance prediction by BBN codes is now limited to a few percents because of the uncertainties on the $\mathrm{D}(\mathrm{p}, \gamma)^{3} \mathrm{He}, \mathrm{D}(\mathrm{d}, \mathrm{n})^{3} \mathrm{He}$ and $\mathrm{D}(\mathrm{d}, \mathrm{p})^{3} \mathrm{H}$ thermonuclear reaction rates (Coc et al. 2015; Di Valentino et al. 2014). Ongoing experiments (Gustavino, 2017) from LUNA at the Gran Sasso underground facility, supplemented by theoretical works (Marcucci et al., 2016) are expected to improve the situation. Here, we concentrated on the prediction of ${ }^{4} \mathrm{He}$ primordial abundance. Uncertainties on $Y_{\mathrm{P}}$ related to experimental data come from the neutron lifetime $879.5( \pm 0.8) \mathrm{s}$ (but that may be affected by systematic uncertainties (Patrignani and Particle Data Group, 2016 and 2017 update) ), leading to a $\Delta Y_{\mathrm{P}}^{(4)}=1.7$ (Eq. 7) uncertainties and from the ${ }^{1} \mathrm{H}(\mathrm{n}, \gamma)^{2} \mathrm{H}, \mathrm{D}(\mathrm{d}, \mathrm{n})^{3} \mathrm{He}$ and $\mathrm{D}(\mathrm{d}, \mathrm{p})^{3} \mathrm{H}$ reaction rates $(\sim 1 \%$ factor uncertainty (Ando et al. 2006, Gómez Iñesta et al., 2017)) leading to $\Delta Y_{\mathrm{P}}^{(4)} \lesssim 0.5$ in total (Eq. 143 . These uncertainties are small, compared with the observational uncertainty of $\Delta Y_{\mathrm{P}}^{(4)}=40$ (Eq. 4). However, the predicted ${ }^{4} \mathrm{He}$ primordial abundance includes corrections to the "bare" weak rates: zero-temperature radiative corrections, finite nucleon mass corrections, finite temperature radiative corrections, weak-magnetism, QED plasma effects and incomplete neutrino decoupling that, in total, shift the abundance by $\delta Y_{\mathrm{P}}^{(4)}=44.7$ (Table $\mathrm{V}$ ), i.e. larger than the uncertainties.

It is thus of the utmost importance to precisely calculate all these corrections, in order to limit theoretical uncertainties. Here, they are for the first time all included and calculated in a self consistent way allowing to take into account the correlations between them. In addition, it was verified that all satisfy detailed balance, a crucial point since it directly affects the neutron/proton number ratio, and hence the ${ }^{4} \mathrm{He}$ abundance. Table $\mathrm{V}$ details the contributions of these corrections to the ${ }^{4} \mathrm{He}$, $\mathrm{D},{ }^{3} \mathrm{He}$ and ${ }^{7} \mathrm{Li}$ primordial abundance. We did not calculate the effect of incomplete neutrino decoupling, but use the results of Pisanti et al. (2008). This amounts to ignoring the spectral distortions, but this affects $Y_{\mathrm{P}}^{(4)}$ by approximately less than one unit. However we find results for incomplete neutrino decoupling effects which are very similar to Grohs et al. (2016), and most notably we find a coherence on the sign of light element abundances variations which is different from Mangano et al. (2005). Given the coupling we find with finite nucleon mass effect, this is the very last correction which requires careful evaluation to fully settle the weak rates corrections.

In this work, we have been using a network of $\approx 400$ nuclear reactions (and their reverse), from neutron to sodium to encompass the light element big bang nucleosynthesis up to the CNO isotopes (Coc et al. 2012). We used the most up to date reaction rates, and in particular those involved in deuterium production (Gómez Iñesta et al., 2017; Iliadis et al. 2016), but even more impor- 
tant, the weak rates with their carefully calculated corrections. Hence, we claim that our predicted abundances (Table VII) are the most accurate to date, not only for $\mathrm{D},{ }^{3} \mathrm{He},{ }^{7} \mathrm{Li}$ and $\mathrm{CNO}$ isotopes but also for ${ }^{4} \mathrm{He}$. Our predicted deuterium and ${ }^{4} \mathrm{He}$ primordial abundances are in agreement with observations, within error bars (Fig. 26 and Table VIII), and in particular, there is no need for extra relativistic degrees of freedom (i.e. $N_{\nu}>3$ ). Our predicted lithium abundance remains a factor of $\approx 3$ above the Spite plateau. The solution of this problem probably involves several mechanisms including stellar depletion and possibly some physics beyond the standard model. Finally, our prediction of CNO abundance (Table VII) does not completely rule out the possibility that they may influence the evolution of some of the first, Population III, stars (Ekström et al. 2008).

Last, but not least, we provide at http://www2.iap. fr/users/pitrou/primat.htm, a freely available Mathematica code that includes all the physics discussed in this work. We expect that it will be used and hopefully modified in order to include new physics (see e.g. Iocco et al. (2009); Mathews and Kusakabe (2017)), or just to update some reaction rates ${ }^{20}$.

\section{Acknowledgments}

We are indebted to our collaborators on these topics: Pierre Descouvemont, Brian Fields, Stéphane Goriely, Faïrouz Hammache, Christian Iliadis, Keith Olive, Patrick Petitjean. C.P. thanks A. Sirlin and W. J. Marciano for discussions on radiative corrections and neutron lifetime, D. Seckel for comments on (Seckel 1993), and G. Lavaux for discussions on Bayesian inference. We also thank warmly Monique and François Spite for their kind help regarding the Lithium data, and Maxim Pospelov for discussions on radiative corrections.

\section{Appendix A: Thermodynamics}

\section{Thermodynamical quantities}

From the distribution function of a given species, it is possible to define macroscopic quantities such as number density $n$, energy density $\rho$, and pressure $P$. Due to the isotropy of the FL spacetime, the distribution function depends only on the magnitude of spatial momenta, that is it is of the form $f(t, p)$. Furthermore isotropy implies that there is no anisotropic stress. Omitting the time

20 They can be provided in tabular form, in a format similar to the Starlib one (Sallaska et al. 2013) at https://starlib.github. io/Rate-Library/, or as (possibly parameter dependent) analytical formulae. dependence to alleviate the notation, we define

$$
\begin{aligned}
n & =g \int f(p) \frac{4 \pi p^{2} \mathrm{~d} p}{(2 \pi)^{3}} \\
\rho & =g \int f(p) E \frac{4 \pi p^{2} \mathrm{~d} p}{(2 \pi)^{3}} \\
P & =g \int f(p) \frac{p^{2}}{3 E} \frac{4 \pi p^{2} \mathrm{~d} p}{(2 \pi)^{3}}
\end{aligned}
$$

with $E=\sqrt{p^{2}+m^{2}}$ and $g$ the number of spin degrees of freedom of the species considered ( $g=1$ for neutrinos and $g=2$ for all other species). Using $E \mathrm{~d} E=p \mathrm{~d} p$, and given that both Fermi-Dirac and Bose-Einstein distributions are given as functions of $E$ and not $p$, it is often more convenient to rewrite these expressions as

$$
\begin{aligned}
n & =\frac{g}{2 \pi^{2}} \int f(E) p E \mathrm{~d} E \\
\rho & =\frac{g}{2 \pi^{2}} \int f(E) p E^{2} \mathrm{~d} E \\
P & =\frac{g}{6 \pi^{2}} \int f(E) p^{3} \mathrm{~d} E
\end{aligned}
$$

with $p=\sqrt{E^{2}-m^{2}}$.

Bose-Einstein and Fermi-Dirac with statistical physics convention for chemical potential are defined as

$$
g_{T, \mu}^{ \pm}(E) \equiv \frac{1}{\mathrm{e}^{\frac{E-\mu}{T}} \pm 1}
$$

with upper (lower) sign for fermions (bosons). For these distributions we find from Eqs. A2

$$
\begin{aligned}
n(t) & =\frac{g T^{3}}{2 \pi^{2}} I_{ \pm}^{(1,1)}(x, \xi) \\
\rho(t) & =\frac{g T^{4}}{2 \pi^{2}} I_{ \pm}^{(2,1)}(x, \xi) \\
P(t) & =\frac{g T^{4}}{6 \pi^{2}} I_{ \pm}^{(0,3)}(x, \xi)
\end{aligned}
$$

with $x \equiv m / T$ and $\xi \equiv \mu / T$ and the integrals

$$
\begin{aligned}
I_{ \pm}^{(m, n)}(x, y) & =\int_{x}^{\infty} \frac{u^{m}\left(u^{2}-x^{2}\right)^{n / 2}}{\mathrm{e}^{u-y} \pm 1} \mathrm{~d} u, \quad \text { (A5a) } \\
& =\int_{0}^{\infty} \frac{\left(v^{2}+x^{2}\right)^{(m-1) / 2} v^{n+1}}{\mathrm{e}^{\sqrt{v^{2}+x^{2}}-y} \pm 1} \mathrm{~d} v \cdot(\mathrm{A} 5 \mathrm{~b})
\end{aligned}
$$

When the chemical potential can be neglected $(\xi \ll 1)$ we also use the notation

$$
I_{ \pm}^{(m, n)}(x) \equiv I_{ \pm}^{(m, n)}(x, 0) .
$$

Finally when the mass can also be neglected $(x \ll 1)$ we define

$$
I_{ \pm}^{(m, n)} \equiv I_{ \pm}^{(m, n)}(0,0),
$$

whose most useful values are reported in table IX. When 
TABLE IX Integrals involved in the expressions of thermodynamical quantities when $x \ll 1$ and $\xi \ll 1$.

\begin{tabular}{ccc}
\hline & Bosons $(-)$ & Fermions $(+)$ \\
\hline$I_{ \pm}^{(0,1)}$ & $\pi^{2} / 6$ & $\pi^{2} / 12$ \\
$I_{ \pm}^{(1,1)}$ & $2 \zeta(3)$ & $3 \zeta(3) / 2$ \\
$I_{ \pm}^{(2,1)}$ & $\pi^{4} / 15$ & $7 \pi^{4} / 120$ \\
$I_{ \pm}^{(0,3)}$ & $\pi^{4} / 15$ & $7 \pi^{4} / 120$ \\
\hline
\end{tabular}

the temperature is much smaller than the mass of the particles, that is for $x=m / T \gg 1$, we find the approximate relations

$$
\begin{aligned}
n & \simeq g T^{3}\left(\frac{x}{2 \pi}\right)^{3 / 2} \mathrm{e}^{\xi-x} \\
\rho & \simeq\left(m+\frac{3}{2} T\right) n .
\end{aligned}
$$

\section{Chemical potential of electrons}

In chemical equilibrium $\mu_{e^{-}}=-\mu_{e^{+}} \equiv \mu_{e}$ because of reactions $2 \gamma \leftrightarrow e^{+}+e^{-}$. Hence the net negative charge density in electrons and positrons is

$$
n_{e^{-}}-n_{e^{+}}=\frac{2 T^{3}}{2 \pi^{2}}\left[I_{+}^{(1,1)}(x, \xi)-I_{+}^{(1,1)}(x,-\xi)\right]
$$

with $x \equiv m_{e} / T$ and $\xi \equiv \mu_{e} / T$. The chemical potential of electrons and positrons must adapt to ensure the electric neutrality of the Universe, hence it is constrained by

$$
n_{e^{-}}-n_{e^{+}}=n_{p}
$$

Let us estimate the chemical potential at the end of BBN for simplicity. Indeed, since $\xi$ increases with time, it is enough to check that its final value at the end of BBN is small. We use therefore the final value of baryon-tophoton ratio $\eta$ [defined in Eq. (36)] and use that the baryonic matter is essentially in protons and ${ }^{4} \mathrm{He}$ nuclei. We find

$$
n_{e^{-}}-n_{e^{+}}=\left(1-Y_{\mathrm{P}} / 2\right) \eta_{0} \bar{n}_{\gamma} T^{3} .
$$

In that limit, the electric charge density of electrons and positrons is given by

$$
n_{e^{-}}-n_{e^{+}} \simeq 4 T^{3}\left(\frac{x}{2 \pi}\right)^{3 / 2} \mathrm{e}^{-x} \sinh \xi .
$$

Combining these two equations we obtain

$$
\xi \simeq \operatorname{Arcinsh}\left(\frac{\eta\left(1-Y_{\mathrm{P}} / 2\right) \mathrm{e}^{x} \sqrt{2} \zeta(3)}{\sqrt{\pi} x^{3 / 2}}\right) .
$$

The evolution of the electrons chemical potential is depicted in Fig. 30 $\xi=\mu_{e} / T$ stays much below $10^{-5}$ whenever $T>5 \times 10^{8} \mathrm{~K}$. For lower temperatures, $\xi$ rises but it is only because electron-positron annihilations are nearly complete and the chemical potential adapts so that the relic density of electrons is equal to the number of protons. That is, whenever the chemical potential of electrons rises at low temperature, the amount of electrons is insignificant due to the low value of the baryon to photon ratio. Hence for all practical purposes, from Eq. 20 we deduce that whenever there is no heat exchange with the neutrinos, the total entropy of the plasma is conserved, that is $\dot{S}_{\mathrm{pl}}=\mathrm{d}\left(s_{\mathrm{pl}} a^{3}\right) / \mathrm{d} t=0$.

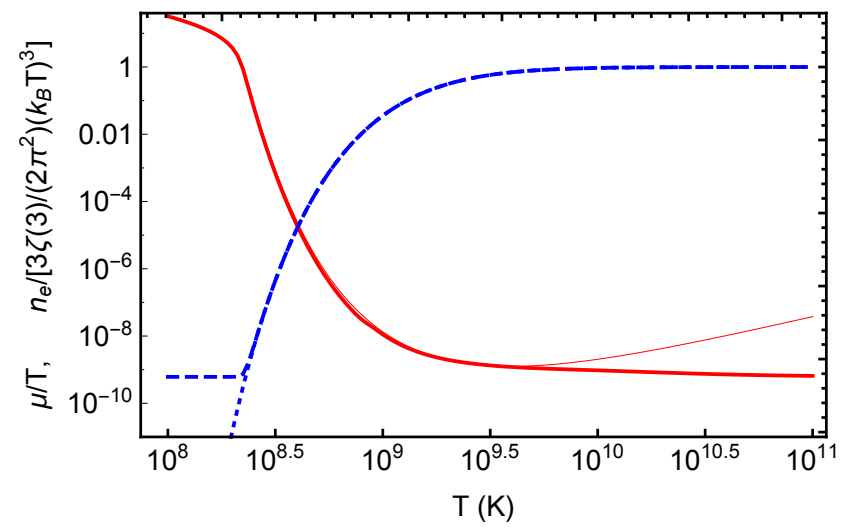

FIG. 30 Continuous red lines : Thick line is $\mu_{e} / T$ computed exactly from Eq. A10, thin line is the approximation A13. Dashed blue line : $2 \pi^{2} /[3 \zeta(3)] n_{e} /\left(k_{B} T\right)^{3}$ with the chemical potential taken into account. Dotted blue line : $2 \pi^{2} /[3 \zeta(3)] n_{e} /\left(k_{B} T\right)^{3}$ with vanishing chemical potential.

\section{Nucleons at thermodynamical equilibrium}

The chemical potential of neutrons and protons are not negligible individually, and they are constrained to give the number density in the low temperature limit A8a. However the difference $\mu_{n}-\mu_{p}$ is negligible with respect to $T$. Indeed we can neglect $\xi_{e} \equiv \mu_{e} / T$ (see $\S \mathrm{A} .2$ ) and assuming it is also the case for neutrinos (see \$VI.C for degenerate neutrinos), and given that at equilibrium we have necessarily

$$
\mu_{n}+\mu_{\nu}=\mu_{p}+\mu_{e} \Rightarrow \mu_{n}-\mu_{p}=\mu_{e}-\mu_{\nu},
$$

then we deduce $\mu_{n}-\mu_{p} \ll 1$.

Hence from $\mathrm{A} 8 \mathrm{a}$ we obtain that the neutron to proton ratio is

$$
\frac{n_{n}}{n_{p}}=\left(\frac{m_{n}}{m_{p}}\right)^{3 / 2} \mathrm{e}^{-\frac{\Delta}{T}} \simeq \mathrm{e}^{-\frac{\Delta}{T}}\left(1+\frac{3 \Delta}{2 m_{N}}\right) .
$$

It is possible to determine the chemical potential of neutrons and protons from the expression A8a and the baryon-to-photon number ratio $\eta$. For instance for protons, we must have

$$
\bar{n}_{p} \equiv \frac{n_{p}}{T^{3}}=2\left(\frac{x_{p}}{2 \pi}\right)^{3 / 2} \mathrm{e}^{\xi_{p}-x_{p}}=\eta \frac{n_{p}}{n_{\mathrm{b}}} \bar{n}_{\gamma}=Y_{p} \eta \bar{n}_{\gamma}
$$


with $x_{p} \equiv m_{p} / T$ and $\xi_{p}=\mu_{p} / T$, which can be used to solve for $\xi_{p}$. In particular the distribution function of protons is approximately given by

$$
f_{p}(p) \simeq \mathrm{e}^{\frac{\mu_{p}-E}{T}} \simeq \exp \left(\xi_{p}-x_{p}-\frac{p^{2}}{2 m_{p} T}\right)
$$

and thus it is approximated by the Maxwellian distribution

$$
\begin{aligned}
f_{p}(p) & \simeq \frac{n_{p}}{2}\left(\frac{2 \pi}{m_{p} T}\right)^{3 / 2} \exp \left(-\frac{p^{2}}{2 m_{p} T}\right) \\
& \simeq Y_{p} \frac{\eta \zeta(3) \sqrt{8}}{\sqrt{\pi}}\left(\frac{T}{m_{p}}\right)^{3 / 2} \exp \left(-\frac{p^{2}}{2 m_{p} T}\right) .
\end{aligned}
$$

From this last equation we deduce that $f_{p}(p) \ll 1$, justifying that we neglect Pauli-blocking effects in reactions rates for protons (and similarly for neutrons).

The distribution function for an isotope $i$ is exactly similar with the obvious replacement $Y_{p} \rightarrow Y_{i}, n_{p} \rightarrow n_{i}$ and $m_{p} \rightarrow m_{i}$. The distribution of velocities is defined as (omitting the $i$ index of the isotope considered)

$$
\phi_{\mathrm{MB}}(v) \mathrm{d} v \equiv \frac{1}{n} \frac{\mathrm{d} n}{\mathrm{~d} p} \mathrm{~d} p=\frac{2}{n} f(p) \frac{4 \pi p^{2} \mathrm{~d} p}{(2 \pi)^{3}},
$$

where $m v=p$, and using the kinetic energy $E \equiv$ $\frac{1}{2} m v^{2} \Rightarrow \mathrm{d} E=m v \mathrm{~d} v$, it takes the form given in Eq. (123).

\section{Abundances at nuclear statistical equilibrium}

At nuclear statistical equilibrium (NSE), a given isotope $i$ is at chemical equilibrium with its nucleons, hence the relation between chemical potentials

$$
\mu_{i}=Z_{i} \mu_{p}+\left(A_{i}-Z_{i}\right) \mu_{n} .
$$

From Eq. A8a

$$
n_{i}=g_{i}\left(\frac{m_{i} T}{2 \pi}\right)^{3 / 2} \mathrm{e}^{\xi_{i}-x_{i}}, \quad g_{i} \equiv 2 s_{i}+1,
$$

where $s_{i}$ is the spin of species $i$. Using Eq. A20 and A21, we get

$$
\begin{aligned}
n_{i}^{\mathrm{NSE}}= & \frac{g_{i} m_{i}^{3 / 2}}{2^{A_{i}}}\left(\frac{n_{p}}{m_{p}^{3 / 2}}\right)^{Z_{i}}\left(\frac{n_{n}}{m_{n}^{3 / 2}}\right)^{A_{i}-Z_{i}} \\
& \times\left(\frac{2 \pi}{T}\right)^{\frac{3\left(A_{i}-1\right)}{2}} \mathrm{e}^{B_{i} / T}
\end{aligned}
$$

where we defined the binding energy

$$
B_{i} \equiv Z_{i} m_{p}+\left(A_{i}-Z_{i}\right) m_{n}-m_{i} .
$$

Using (36), the abundances are then given at NSE by

$$
\begin{aligned}
Y_{i}^{\mathrm{NSE}}= & g_{i} \zeta(3)^{A_{i}-1} 2^{\frac{3 A_{i}-5}{2}} \pi^{\frac{1-A_{i}}{2}}\left(\frac{m_{i} T^{A_{i}-1}}{m_{p}^{Z_{i}} m_{n}^{A_{i}-Z_{i}}}\right)^{3 / 2} \\
& \times \eta^{A_{i}-1} Y_{p}^{Z_{i}} Y_{n}^{A_{i}-Z_{i}} \mathrm{e}^{B_{i} / T} .
\end{aligned}
$$

We check in particular that for neutrons and protons $B_{n}=B_{p}=0$ and $A_{n}=A_{p}=1$, so we get the tautological relation $Y_{n}^{\mathrm{NSE}}=Y_{n}$ and $Y_{p}^{\mathrm{NSE}}=Y_{p}$. In order to obtain the NSE values from A24, we use Audi et al. (2017) for the masses and spins of nuclear elements. In Fig. 31. we plot jointly the abundances of the main isotopes together with their NSE values to check that they are equal at high temperatures.

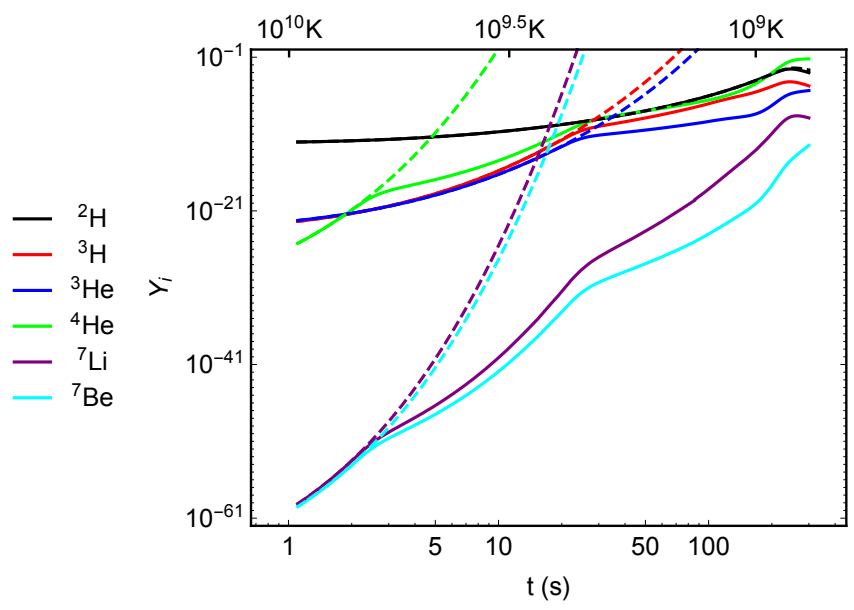

FIG. 31 Evolution of the first elements abundances in solid lines, together with the nuclear statistical equilibrium values in dashed lines. The deuterium abundance stays very close to its NSE value until the time it is more efficiently destroyed than formed around $t \simeq 200 \mathrm{~s}$.

\section{Appendix B: Weak reactions rates}

In this section, we gather all the technicalities required to obtain the theoretical forms of the weak-rates including the most relevant corrections.

\section{General expressions}

Let us consider first the reactions with neutrons in the initial state. Throughout this section we use the mostly minus metric signature since it is the most common in particle physics. The general expression for each of the three reactions involved is of the form (Fidler and Pitrou. 2017; Lopez et al. 1997

$$
\begin{aligned}
& n_{n} \Gamma=\int \Pi_{i}\left[\mathrm{~d}^{3} \mathbf{p}_{i}\right](2 \pi)^{4} \delta^{4}\left(\underline{p}_{n}-\underline{p}_{p}+\alpha_{\nu} \underline{p}_{\nu}+\alpha_{e} \underline{p}_{e}\right) \\
& \quad \times|M|^{2} f_{n}\left(E_{n}\right)\left[1-f_{p}\left(E_{p}\right)\right] f_{\nu}\left(\alpha_{\nu} E_{\nu}\right) f_{e}\left(\alpha_{e} E_{e}\right)
\end{aligned}
$$

where the $p_{i}$ with $i=n, p, e, \nu$ are the four-momenta of particles, and we used the compact notation for the relativistic volume element

$$
\left[\mathrm{d}^{3} \mathbf{p}\right] \equiv \frac{\mathrm{d}^{3} \mathbf{p}}{2 E(2 \pi)^{3}}=\frac{4 \pi p^{2} \mathrm{~d} p}{2 E\left(2 \pi^{3}\right)} .
$$


The factor $\alpha_{\nu}$ is +1 if the neutrino is in the initial state and -1 otherwise with a similar definition for $\alpha_{e}$ and the electron or positron. In (B1), it is apparent that the Dirac function ensures energy and momentum conservation. For a Fermi-Dirac distribution

$$
g(-E)=1-g(E)
$$

implying that the coefficients $\alpha_{e}, \alpha_{\nu}$ also ensure that either it is the distribution function which appears if the lepton is in the initial state, or the Pauli-blocking factor if it is in the final state.

A first simplification consists in neglecting the Pauliblocking factor of the final state proton due to the very low value of the baryon-to-photon ratio [see Eq. A18]. Having no dependence on the distribution function of the final protons, we can simplify Eq. (B1) further by performing the integral on spatial momenta of protons, using the spatial part of the Dirac function. The reaction rates reduces to

$$
\begin{aligned}
n_{n} \Gamma= & \int \frac{\mathrm{d}^{3} \mathbf{p}_{n} \mathrm{~d}^{3} \mathbf{p}_{e} \mathrm{~d}^{3} \mathbf{p}_{\nu}}{2^{4}(2 \pi)^{8}} \delta\left(E_{n}-E_{p}+\alpha_{e} E_{e}+\alpha_{\nu} E_{\nu}\right) \\
& \times \frac{|M|^{2}}{E_{n} E_{p} E_{e} E_{\nu}} f_{n}\left(E_{n}\right) f_{\nu}\left(\alpha_{\nu} E_{\nu}\right) f_{e}\left(\alpha_{e} E_{e}\right)
\end{aligned}
$$

where the proton momentum and energies are related by

$$
\mathbf{p}_{p}=\mathbf{p}_{n}+\alpha_{\nu} \mathbf{p}_{\nu}+\alpha_{e} \mathbf{p}_{e}, \quad E_{p}=\sqrt{\mathbf{p}_{p} \cdot \mathbf{p}_{p}+m_{p}^{2}} .
$$

For a given reaction, $|M|^{2}$ is the corresponding matrixelement of the weak interaction summed over all initial and final states, computed from the interaction Hamiltonian. For the reactions (68), the relevant part of the interaction Hamiltonian is a coupling of weak currents in the form

$$
\mathcal{H}_{I}=\frac{G_{F}}{\sqrt{2}} J_{e \nu}^{\mu} J_{p n, \mu}
$$

where $G_{F}$ is the Fermi constant. The electron-neutron weak current is purely left chiral, and of the form

$$
J_{e \nu}^{\mu}=\overline{\boldsymbol{\nu}} \gamma^{\mu}\left(1-\gamma^{5}\right) \mathbf{e} .
$$

e and $\boldsymbol{\nu}$ are the fermionic quantum fields of electrons and neutrinos, and the $\gamma^{\mu}$ are the matrices of the Clifford algebra [we use the conventions of (Fidler and Pitrou, 2017)]. The proton-neutron current is of the form (Ivanov et al. 2013, Eqs. 1 and A2)

$$
J_{p n}^{\mu}=\cos \theta_{\mathrm{C}} \overline{\mathbf{p}}\left(\gamma^{\mu}\left(1-g_{A} \gamma^{5}\right)+\mathrm{i} \frac{f_{\mathrm{wm}}}{m_{N}} 2 \Sigma^{\mu \nu} q_{\nu}\right) \mathbf{n}
$$

where $\mathbf{p}$ and $\boldsymbol{n}$ are the Fermionic quantum fields of protons and neutrons and $2 \Sigma^{\mu \nu} \equiv \mathrm{i} / 2\left(\gamma^{\mu} \gamma^{\nu}-\gamma^{\nu} \gamma^{\mu}\right) . g_{A}$ is the axial current constant for nucleons (also sometimes written $C_{A} / C_{V}$ or $\lambda$ in the literature), and $f_{\text {wm }}$ is the weak magnetism constant whose numerical value is given by (Horowitz, 2002, Horowitz and Li, 2000, Ivanov et al. 2013)

$$
f_{\mathrm{wm}}=\frac{\mu_{p}-\mu_{n}}{2} \simeq \frac{1.793-(-1.913)}{2} \simeq 1.853 .
$$

$\theta_{\mathrm{C}}$ is the Cabbibo-Kobayashi-Maskawa (CKM) angle $\left(\cos \theta_{\mathrm{C}}\right.$ also noted $V_{u d}$ in the literature), $q^{\mu}$ is the nucleon four-momentum transfer, that is the difference between the final nucleon four-momentum and the initial one. The most recent numerical values for the constants in weak interactions are gathered in appendix D,

It is possible to show that from Eqs. (B6)-(B8) the matrix element is of the form [see e.g. (Fidler and Pitrou, 2017)]

$$
\frac{|M|^{2}}{2^{7} G_{F}^{2}}=c_{L L} \mathcal{M}_{L L}+c_{R R} \mathcal{M}_{R R}+c_{L R} \mathcal{M}_{L R}
$$

with the coupling factors

$$
\begin{aligned}
c_{L L} & \equiv \frac{\left(1+g_{A}\right)^{2}}{4} \\
c_{R R} & \equiv \frac{\left(1-g_{A}\right)^{2}}{4} \\
c_{L R} & \equiv \frac{g_{A}^{2}-1}{4} .
\end{aligned}
$$

If weak interactions for nucleons were purely left-chiral, that is with $g_{A}=1$, only the $\mathcal{M}_{L L}$ would contribute. $\mathcal{M}_{R R}$ contains the contribution of the right-chiral part of the interaction, and $\mathcal{M}_{L R}$ is an interference between left and right chiral contributions.

Let us ignore first the contribution from weakmagnetism that are investigated further in $\S$ B.4. We then find

$$
\begin{aligned}
\mathcal{M}_{L L} & =\left(\underline{p}_{n} \cdot \underline{p}_{\nu}\right)\left(\underline{p}_{p} \cdot \underline{p}_{e}\right) \\
\mathcal{M}_{R R} & =\left(\underline{p}_{n} \cdot \underline{p}_{e}\right)\left(\underline{p}_{p} \cdot \underline{p}_{\nu}\right) \\
\mathcal{M}_{L R} & =m_{p} m_{n}\left(\underline{p}_{\nu} \cdot \underline{p}_{e}\right) .
\end{aligned}
$$

It is worth noting that all weak reactions 68 have in fact the same matrix element. First the three reactions with a neutron in the initial state are obtained by crossing symmetry. Crossing symmetry for the electron amounts for instance to the formal replacement $p_{e} \rightarrow-p_{e}$ with an overall minus sign (Fidler and Pitrou, 2017) and it is straightforward to check that this leaves the matrix element invariant. The same property arises obviously for neutrinos. Second, the three reverse reactions (with an initial proton and a final neutron) are obtained by time reversal, and since there is no $\mathrm{CP}$ violation at this level, there is also a time-reversal symmetry so as to ensure the CPT symmetry. Hence all reverse rates have exactly the same matrix element.

\section{Fokker-Planck expansion}

The integral in (B4) is 8-dimensional when one removes the Dirac function. Due to the isotropy of all distribu- 
tions, this can be reduced to a 5-dimensional integral. This is the method followed by Lopez et al. (1997). Here we follow a much simpler route by performing a Fokkerplanck expansion in the energy transferred in reactions. Even though our method is different from Seckel (1993), it follows a method which is similar in spirit. As we shall see, this results in one-dimensional integrals which are much faster to evaluate.

At low temperature, it is enough to assume that nucleons follow an isotropic Maxwellian distribution of velocities at the plasma temperature $T$ given by Eq. (A18). Hence the following integrals are obtained

$$
\begin{aligned}
2 \int f_{N}(\mathbf{p}) \frac{\mathrm{d}^{3} \mathbf{p}}{(2 \pi)^{3}} & =n_{N}, \\
2 \int f_{N}(\mathbf{p}) \frac{p^{i}}{m_{N}} \frac{\mathrm{d}^{3} \mathbf{p}}{(2 \pi)^{3}} & =0, \\
2 \int f_{N}(\mathbf{p}) \frac{p^{i} p^{j}}{m_{N}^{2}} \frac{\mathrm{d}^{3} \mathbf{p}}{(2 \pi)^{3}} & =\frac{T}{m_{N}} \delta^{i j} n_{N} .
\end{aligned}
$$

In particular contracting with $\delta_{i j}$ we recover the expression for the pressure of nucleons in the low temperature limit

$$
P_{N}=2 \int f_{N}(\mathbf{p}) \frac{p^{2}}{3 m_{N}} \frac{\mathrm{d}^{3} \mathbf{p}}{(2 \pi)^{3}}=T n_{N} .
$$

For electron or neutrino distributions, since we have assumed isotropy, we deduce the property

$$
\int g(E) p^{\alpha} E^{\beta} p^{i} p^{j} \frac{\mathrm{d}^{3} \mathbf{p}}{(2 \pi)^{3}}=\frac{\delta^{i j}}{3} \int g(E) p^{\alpha+2} E^{\beta} \frac{\mathrm{d}^{3} \mathbf{p}}{(2 \pi)^{3}}
$$

where $\alpha$ and $\beta$ are some numbers. From isotropy we also find that

$$
\int g(E) p^{\alpha} E^{\beta} p^{i} \frac{\mathrm{d}^{3} \mathbf{p}}{(2 \pi)^{3}}=0 .
$$

Hence for all practical purposes, we can perform the replacements

$$
p^{i} p^{j} \rightarrow p^{2} \delta^{i j} / 3, \quad p^{i} \rightarrow 0
$$

on all species, resulting in great simplifications.

We assess the importance of corrections to the Born approximations as explained in $\S$ III.G. that is in powers of $\epsilon \equiv T / m_{N}$. To evaluate the order of each term, we consider that the momentum or energies of neutrinos are of order $T \sim \Delta$, that is factors of the type $E_{e} / m_{N}$ or $E_{\nu} / m_{N}$ are of order $\epsilon$. Furthermore, from (B13) a factor $\mathbf{p}_{n} / m_{n}$ is of order $\sqrt{T / M} \sim \sqrt{\Delta / M}$ and thus $\sqrt{\epsilon}$. However since only even powers of the spatial momentum of nucleons must appear [see Eqs. (B13)], we shall encounter terms of the type $\left|\mathbf{p}_{p} / m_{n}^{2}\right|$ which are of order $\epsilon$.

The Fokker-Planck expansion consists in expanding the energy difference between the nucleons, $E_{n}-E_{p}$ around the lowest order value $\Delta=m_{n}-m_{p}$. Keeping only the lowest corrections this expansion reads

$$
\begin{aligned}
E_{n}-E_{p}=\Delta+\delta Q_{1}+\delta Q_{2}+\delta Q_{3} \\
\delta Q_{1} \equiv-\frac{\mathbf{p}_{n} \cdot \mathbf{q}}{m_{N}} \\
\delta Q_{2} \equiv-\frac{|\mathbf{q}|^{2}}{2 m_{N}} \\
\delta Q_{3} \equiv \frac{\left|\mathbf{p}_{n}\right|^{2}}{2}\left(\frac{1}{m_{n}}-\frac{1}{m_{p}}\right) \simeq-\frac{\left|\mathbf{p}_{n}\right|^{2} \Delta}{2 m_{N}^{2}}
\end{aligned}
$$

where $\mathbf{q} \equiv \mathbf{p}_{p}-\mathbf{p}_{n}=\alpha_{\nu} \mathbf{p}_{\nu}+\alpha_{e} \mathbf{p}_{e}$ is the spatial momentum transferred. The first term in (B18) is the lowest order, or Born approximation, that is the only appearing when considering the infinite nucleon mass approximation. The second term is an order $\sqrt{\epsilon}$ correction, and the third term is an order $\epsilon$ correction. Finally the last term is of order $T \Delta / m_{N}$ so it is an order $\epsilon$ correction as well. It is the only corrective term for which it is crucial to take into account the difference of mass between neutrons and protons. Using Eq. (B18), we expand the Dirac delta function on energies as

$$
\begin{aligned}
\delta\left(E_{n}-E_{p}+\alpha_{e} E_{e}+\alpha_{\nu} E_{\nu}\right) & \simeq \\
\delta(\Sigma)+\delta^{\prime}(\Sigma)\left(\sum_{i=1}^{3} \delta Q_{i}\right) & +\frac{1}{2} \delta^{\prime \prime}(\Sigma)\left(\delta Q_{1}\right)^{2}
\end{aligned}
$$

where $\Sigma \equiv \Delta+\alpha_{e} E_{e}+\alpha_{\nu} E_{\nu}$.

We must then expand the matrix element and the energies appearing in Eq. (B4). It proves much easier to expand all these contributions together. Furthermore, whenever a term is already of order $\epsilon$, we know that it should multiply only the Born term of the expansion (B20), so we can apply the simplification rule (B17). With this method we find

$$
\begin{aligned}
& \frac{\mathcal{M}_{L L}}{\Pi_{i} E_{i}} \rightarrow 1-\frac{\mathbf{p}_{n}}{m_{N}} \cdot\left(\frac{\mathbf{p}_{e}}{E_{e}}+\frac{\mathbf{p}_{\nu}}{E_{\nu}}\right)-\frac{\alpha_{\nu}\left|\mathbf{p}_{\nu}\right|^{2}}{m_{N} E_{\nu}}(\mathrm{B} 21 \mathrm{a}) \\
& \frac{\mathcal{M}_{R R}}{\Pi_{i} E_{i}} \rightarrow 1-\frac{\mathbf{p}_{n}}{m_{N}} \cdot\left(\frac{\mathbf{p}_{e}}{E_{e}}+\frac{\mathbf{p}_{\nu}}{E_{\nu}}\right)-\frac{\alpha_{e}\left|\mathbf{p}_{e}\right|^{2}}{m_{N} E_{e}}(\mathrm{~B} 21 \mathrm{~b}) \\
& \frac{\mathcal{M}_{L R}}{\Pi_{i} E_{i}} \rightarrow\left(1-\frac{\left|\mathbf{p}_{n}\right|^{2}}{m_{N}^{2}}\right)\left(1-\frac{\mathbf{p}_{e} \cdot \mathbf{p}_{\nu}}{E_{e} E_{\nu}}\right) \cdot \quad(\mathrm{B} 21 \mathrm{c})
\end{aligned}
$$

The second term in Eqs. (B21a) and $\mathrm{B} 21 \mathrm{~b}$ is of order $\sqrt{\epsilon}$ and the last term in these equations is of order $\epsilon$. Hence the second term needs to be coupled with the order $\sqrt{\epsilon}$ term in the Dirac delta expansion (B20) which is $\delta^{\prime}(\Sigma) \delta Q_{1}$, and simplified with the rules (B17).

There are four steps to complete this Fokker-Planck expansion.

1. First, using Eqs. B21 and $\mathrm{B} 20$ in the reaction rates (B4) we perform the integral on the initial neutron momentum with the rules $\mathrm{B} 13$. 
2. Second, we can replace the differential elements for the integral on electron and neutrino momenta with $\mathrm{d}^{3} p \rightarrow 4 \pi p^{2} \mathrm{~d} p$ because we have already performed all angular averages.

3. We are left with a two dimensional integral on the electron and neutrino momentum magnitudes $p_{e}=\left|\mathbf{p}_{e}\right|$ and $p_{\nu}=\left|\mathbf{p}_{\nu}\right|$. Let us note $E_{\nu}=p_{\nu}$ in order to write the result in a easily readable form. Third, we perform the integral on $E_{\nu}$ using the Dirac delta and their derivatives. Whenever a Dirac delta derivative appears, it means that we have to perform integration by parts to convert it into a normal Dirac delta. This will introduce derivatives with respect to the $E_{\nu}$ applied on the neutrino distribution function or Pauli-blocking factor. Also for a given reaction it might appear that the value of $E_{\nu}$ constrained by the Dirac delta is not physical for that reaction if $\alpha_{\nu}=1$ and physical if $\alpha_{\nu}=-1$, or vice-versa. This is the reason why we consider the total reaction rate of the reactions 68a and (68b). Once their rates are added, the Dirac delta automatically selects either the neutrino in the initial state, with the corresponding distribution function, or the neutrino in the final state, with the associated Pauli-blocking factor. Eventually once the rates (68a) and (68b) are added, we might forget about $\alpha_{\nu}$, that is about the position of the neutrino. We need only to compute two rates, one where the electrons is in the initial state [reaction [68c)], and one where it is a positron which is in the final state [the sum of reactions 68a and [68b] ].

4. Finally, we need to determine the procedure to convert the rate with a neutron in the initial state into the reverse rate with a proton in the initial state. Even if the matrix element is the same for all reactions, as explained in $\S$ B.1 the method to perform a finite mass expansion is not symmetric under the interchange $p \leftrightarrow n$. Indeed we chose to expand the momentum of the final nucleon around the initial one, and we remove the integral on the final nucleon momenta. It is apparent on Eqs. (B12) that the electron (resp. neutrino) momentum is contracted with the neutron (resp. proton) in the $L L$ term but this is the opposite in the $R R$ term. Since the coupling factors of these terms are interchanged by the replacement $g_{A} \rightarrow-g_{A}$, we can deduce the rates with an initial proton from those with an initial neutron using the rule $g_{A} \rightarrow-g_{A}$. Obviously the argument of the Dirac delta contains now $E_{p}-E_{n}=-\Delta+\ldots$ instead of $E_{n}-E_{p}=\Delta+\ldots$ so we must also apply the rule $\Delta \rightarrow-\Delta$. Finally when considering a reverse reaction, the electron in the initial state turns into a positron in the final state so we must also apply the rule $E_{e} \rightarrow-E_{e}$, that is change the electron distribution function to a Pauli-blocking factor or vice-versa. These are the rules used extensively in $\S$ III to deduce the reverse rates.

Having sketched the details of the procedure, we are in position to give the results. The Born approximation results are obvious under this expansion. From Eqs. B21 we obtain in the limit

$$
\begin{aligned}
& \frac{\mathcal{M}_{L L}}{\Pi_{i} E_{i}} \simeq \frac{\mathcal{M}_{R R}}{\Pi_{i} E_{i}} \simeq 1, \\
& \frac{\mathcal{M}_{L R}}{\Pi_{i} E_{i}} \simeq\left(1-\frac{\mathbf{p}_{e} \cdot \mathbf{p}_{\nu}}{E_{e} E_{\nu}}\right) .
\end{aligned}
$$

Furthermore in the Dirac expansion $(\mathrm{B} 20)$ we keep only $\delta(\Sigma)$, and the average over electrons and neutrino spatial momenta removes the momentum dependent part of Eq. (B22b). The Born rates are gathered and commented in $\S$ III.B

The first corrections in this Fokker-Planck expansion, and which are due to finite nucleon mass, are reported in the next section.

\section{Finite nucleon mass corrections}

The finite nucleon mass corrections take the form 114 [or 115 if radiative corrections are added]. The function $\chi_{ \pm}^{\mathrm{FN}}$ is 


$$
\begin{aligned}
\chi_{ \pm}^{\mathrm{FM}}\left(E, g_{A}\right)= & \tilde{c}_{L L} \frac{p^{2}}{m_{N} E} g_{\nu}\left(E_{\nu}^{\mp}\right) g(-E)-\tilde{c}_{R R} \frac{E_{\nu}^{\mp}}{m_{N}} g_{\nu}^{(2,0)}\left(E_{\nu}^{\mp}\right) g(-E) \\
& +\left(\tilde{c}_{L L}+\tilde{c}_{R R}\right) \frac{T}{m_{N}}\left(g_{\nu}^{(2,1)}\left(E_{\nu}^{\mp}\right) g(-E) \frac{p^{2}}{E}-g_{\nu}^{(3,1)}\left(E_{\nu}^{\mp}\right) g(-E)\right) \\
+ & \left(\tilde{c}_{L L}+\tilde{c}_{R R}+\tilde{c}_{L R}\right)\left[\frac{T}{2 m_{N}}\left(g_{\nu}^{(4,2)}\left(E_{\nu}^{\mp}\right) g(-E)+g_{\nu}^{(2,2)}\left(E_{\nu}^{\mp}\right) g(-E) p^{2}\right)\right. \\
& \left.+\frac{1}{2 m_{N}}\left(g_{\nu}^{(4,1)}\left(E_{\nu}^{\mp}\right) g(-E)+g_{\nu}^{(2,1)}\left(E_{\nu}^{\mp}\right) g(-E) p^{2}\right)\right] \\
& -\left(\tilde{c}_{L L}+\tilde{c}_{R R}+\tilde{c}_{L R}\right) \frac{3 T}{2}\left(1-\frac{m_{n}}{m_{p}}\right) g_{\nu}^{(2,1)}\left(E_{\nu}^{\mp}\right) g(-E) \\
& +\tilde{c}_{L R}\left[-\frac{3 T}{m_{N}} g_{\nu}^{(2,0)}\left(E_{\nu}^{\mp}\right) g(-E)+\frac{p^{2}}{3 m_{N} E} g_{\nu}^{(3,1)}\left(E_{\nu}^{\mp}\right) g(-E)+\frac{p^{2} T}{3 m_{N} E} g_{\nu}^{(3,2)}\left(E_{\nu}^{\mp}\right) g(-E)\right]
\end{aligned}
$$

where $p=\sqrt{E^{2}-m_{e}^{2}}, E_{\nu}^{\mp}=E \mp \Delta$. We defined the reduced couplings

$$
\begin{aligned}
\tilde{c}_{L L} & \equiv \frac{4}{1+3 g_{A}^{2}} c_{L L} \\
\tilde{c}_{R R} & \equiv \frac{4}{1+3 g_{A}^{2}} c_{R R} \\
\tilde{c}_{L R} & \equiv \frac{4}{1+3 g_{A}^{2}} c_{L R},
\end{aligned}
$$

and the functions [with the notation 275p]

$$
g_{\nu}^{(n, p)}\left(E_{\nu}\right) \equiv \frac{\partial^{p}\left[\left(E_{\nu}\right)^{n} g_{\nu}\left(E_{\nu}\right)\right]}{\partial E_{\nu}^{p}} .
$$

For the neutron beta decay, the expression $(\bar{B} 23)$ for $T=$ 0 gives (with $E_{\nu} \equiv \Delta-E$ here)

$$
\begin{aligned}
& \left.\chi_{+}^{\mathrm{FM}}\left(E, g_{A}\right)\right|_{T=0}=\tilde{c}_{L L} \frac{p^{2} E_{\nu}^{2}}{m_{N} E}+\tilde{c}_{R R} \frac{E_{\nu}^{3}}{m_{N}} \\
& -\left(\tilde{c}_{L L}+\tilde{c}_{R R}+\tilde{c}_{L R}\right) \frac{\left(2 E_{\nu}^{3}+E_{\nu} p^{2}\right)}{m_{N}}+\tilde{c}_{L R} \frac{p^{2} E_{\nu}^{2}}{m_{N} E} .
\end{aligned}
$$
B8 with a factor $q_{\nu} / m_{N}$, with the mom to nucleon being of the order of $T$, it is an order $\epsilon$ correction and thus vanishes at the Born approximation level. The matrix element is corrected by the addition of

$$
\begin{aligned}
\frac{|M|^{2}}{2^{7} G_{F}^{2}} & \supset f_{\mathrm{wm}} g_{A} \frac{m_{n}+m_{p}}{2}\left(\mathcal{M}_{L L}-\mathcal{M}_{R R}\right) \\
& +\frac{f_{\mathrm{wm}}}{2}\left[\frac{m_{n}+m_{p}}{2}\left(\mathcal{M}_{L R}-\mathcal{M}_{L L}-\mathcal{M}_{R R}-\left(\underline{p}_{n} \cdot \underline{p}_{p}\right)\left(\underline{p}_{\nu} \cdot \underline{p}_{e}\right)\right)+m_{p}\left(\underline{p}_{n} \cdot \underline{p}_{e}\right)\left(\underline{p}_{n} \cdot \underline{p}_{\nu}\right)+m_{n}\left(\underline{p}_{p} \cdot \underline{p}_{e}\right)\left(\underline{p}_{p} \cdot \underline{p}_{\nu}\right)\right]
\end{aligned}
$$

where we used the expressions $\mathrm{B} 12$. It is possible to show, although rather tedious, that the contributions of the second line vanish at first order in $\epsilon$. This remarkable simplification has been noticed earlier by Seckel (1993). Hence the weak-magnetism contributes exactly as the axial vector current coupled to the vector current. It is taken into account by changing the coupling constants (B11) to the values (119), modifying accordingly the constants (B24) appearing in the finite nucleon mass corrections.

Only the first two terms of Eq. (B23) can in principle contribute to $\lambda_{0}$ since weak-magnetism does no affect the 
sum $c_{L L}+c_{R R}$ nor $c_{L R}$. However, it is straightforward to show that they result in a vanishing contribution since

$$
\begin{aligned}
\lambda_{0}^{\mathrm{wm}} & \propto \int_{0}^{\sqrt{\Delta^{2}-1}} \mathrm{~d} p p^{2}(E-\Delta)^{2}\left(\frac{p^{2}}{E}+(E-\Delta)\right) \\
& =\frac{1}{3} \int_{m}^{\Delta} \mathrm{d} E \partial_{E}\left[(E-\Delta)^{3}\left(E^{2}-m^{2}\right)^{3 / 2}\right] \\
& =0
\end{aligned}
$$

Note that when the weak-magnetism contribution is coupled with radiative corrections, this result is no longer valid and there is a small change in $\lambda_{0}$ that we give in $\S$ III.H

\section{Mandelstam variables}

Let us define the Mandelstam variables for $\nu+n \rightarrow$ $e+p$.

$$
\begin{aligned}
s & \equiv\left(\underline{p}_{\nu}+\underline{p}_{n}\right)^{2}=\left(\underline{p}_{e}+\underline{p}_{p}\right)^{2} \\
t & \equiv\left(\underline{p}_{\nu}-\underline{p}_{e}\right)^{2}=\left(\underline{p}_{n}-\underline{p}_{p}\right)^{2} \\
u & \equiv\left(\underline{p}_{\nu}-\underline{p}_{p}\right)^{2}=\left(\underline{p}_{n}-\underline{p}_{e}\right)^{2} .
\end{aligned}
$$

The total matrix element $|M|^{2}$ deduced from Eqs. (B6)$(\mathrm{B} 8)$, is the sum of Eqs. (B10) and (B27), where we use the definitions B12. Once expressed with the Mandelstam variables, we checked that it takes the form given in Lopez et al. (1997, App. A) (published version and with $m_{1}=m_{\nu}, m_{2}=m_{n}, m_{3}=m_{e}$ and $\left.m_{4}=m_{p}\right)$, except for a few differences. First, we do not include their term $t_{3}$ because this is second order in finite nucleon mass effects. Hence, we kept only contributions which are linear in $f_{\mathrm{wm}}$. Second, we find that there is a typo in $t_{5}$ of Lopez et al. (1997), since there should be a factor $g_{A}$ instead of $g_{A}^{2}$.

We also performed a comparison with Seckel (1993) and found that it matches our result and the one of Lopez et al. (1997) if we replace its $f_{2}$ by $2 f_{\text {wm }}$ (again ignoring terms which are quadratic in $f_{2}$ and the parameter $f_{p s}$ in Seckel (1993)).

\section{Radiative corrections and Sirlin's universal function}

When considering the effect of radiative corrections, the function $\mathcal{C}$ defined in Eq. 103 is given by

$$
\mathcal{C}\left(E, k_{\max }\right)=4 \ln \frac{m_{Z}}{m_{p}}+\ln \frac{m_{p}}{m_{A}}+2 C+A_{g}+g\left(E, k_{\max }\right)
$$

with

$$
C \simeq 0.891, \quad A_{g} \simeq-0.34, \quad m_{A} \simeq 1.2 \mathrm{GeV} .
$$

$g\left(E, k_{\max }\right)$ is Sirlin's universal function

$$
\begin{aligned}
g\left(E, k_{\max }\right) & =3 \ln \frac{m_{p}}{m_{e}}-\frac{3}{4}+\frac{4}{\beta} L\left(\frac{2 \beta}{1+\beta}\right) \\
& +4[R(\beta)-1]\left[\frac{k_{\max }}{3 E}-\frac{3}{2}+\ln \left(2 \frac{k_{\max }}{m_{e}}\right)\right] \\
& +R(\beta)\left[2\left(1+\beta^{2}\right)+\frac{k_{\max }^{2}}{6 E^{2}}-4 \beta R(\beta)\right]
\end{aligned}
$$

with $\beta=p / E=\sqrt{E^{2}-m_{e}^{2}} / E$ and

$$
R(\beta) \equiv \frac{E}{p} \ln \left(\frac{p+E}{m}\right)=\frac{1}{2 \beta} \ln \left(\frac{1+\beta}{1-\beta}\right)=\frac{\arctan (\beta)}{\beta}
$$

and where the Spence function is defined as

$$
L[x]=\int_{0}^{x} \frac{\ln (1-t)}{t} \mathrm{~d} t .
$$

It is possible to use an expansion of the Spence function. We report it here to correct typos in Dicus et al. (1982) and subsequently in Lopez and Turner (1999); Smith and Fuller (2010)

$$
\begin{aligned}
& \frac{1}{\beta} L\left(\frac{2 \beta}{1+\beta}\right) \simeq-\frac{1}{(1+\beta)^{6}} \times \\
& \left(2+11 \beta+\frac{224}{9} \beta^{2}+\frac{89}{3} \beta^{3}+\frac{1496}{75} \beta^{4}+\frac{596}{75} \beta^{5}+\frac{128}{49} \beta^{6}\right) .
\end{aligned}
$$

Finally in order to obtain even more accurate results, it is also possible to refine the expression for the radiative corrections by using Czarnecki et al. (2004, Eq. 15) which is a resummation of higher order corrections. Unless specified, this is the radiative correction that we use so we report it here.

$$
\begin{aligned}
R\left(E, k_{\max }\right)= & {\left[1+\frac{\alpha_{\mathrm{FS}}}{2 \pi}\left(g\left(E, k_{\max }\right)-3 \ln \frac{m_{p}}{2 \Delta}\right)\right] \quad(\mathrm{B} 35) } \\
& \times\left(L+\frac{\alpha_{\mathrm{FS}}}{\pi} C+\frac{\alpha_{\mathrm{FS}}}{2 \pi} \delta\right) \\
& \times\left[S+\frac{\alpha_{\mathrm{FS}}\left(m_{p}\right)}{2 \pi}\left(\ln \frac{m_{p}}{m_{A}}+A_{g}\right)+\mathrm{NLL}\right]
\end{aligned}
$$

$\alpha_{\mathrm{FS}}\left(m_{p}\right) \simeq \frac{1}{134}, \quad \frac{\alpha_{\mathrm{FS}}}{2 \pi} \delta \simeq-0.00043, \quad \mathrm{NLL} \simeq-10^{-4}$

$$
L \simeq 1.02094, \quad S \simeq 1.02248
$$

\section{Bremsstrahlung}

We review in the next section the bremsstrahlung correction to the neutron decay, also called radiative neutron decay when the photon is detected (Cooper et al. 2010). We then extend it to the other weak processes during BBN. This allows to find the difference between the correct treatment of bremsstrahlung and how it was partially included in the radiative correction factor detailed above in $\S$ B.6. We report subsequently the detailed expression of this bremsstrahlung correction. 


\section{a. Neutron decay}

Let us consider first the bremsstrahlung for the decay of neutron. We note the emitted photon momentum $\underline{k}$. Its spatial part is $\mathbf{k}$, that we decompose into energy $k$ and direction $\hat{\mathbf{k}}$ as $\mathbf{k}=k \hat{\mathbf{k}}$. We note the electron energy $E$ and its spatial momentum $p$ for simplicity. In the infinite nucleon mass approximation, the bremsstrahlung differential decay rate is of the form [see e.g. Ivanov et al. (2013, Eq. B12), Cooper et al. (2010, Eq. 4), Abers et al. (1968, Eq. 6.6) or Ivanov et al. (2017, Eq. A40)]

$$
\begin{aligned}
\frac{\mathrm{d} \Gamma_{n \rightarrow}^{\mathrm{BS}}}{\mathrm{d} E \mathrm{~d} k \mathrm{~d} \mu} & =\frac{\alpha_{\mathrm{FS}} K}{2 \pi} \frac{\beta E_{\nu}^{2}}{k} \\
& \times\left[\frac{\beta^{2}\left(1-\mu^{2}\right)}{(1-\beta \mu)^{2}}\left(1+\frac{k}{E}\right)+\frac{k^{2}}{E^{2}} \frac{1}{1-\beta \mu}\right]
\end{aligned}
$$

where

$$
\mu \equiv \mathbf{p}_{e} \cdot \hat{\mathbf{k}}
$$

is the (cosinus) of the angle between the photon momentum and the electron momentum. The neutrino energy is constrained from energy conservation to be

$$
E_{\nu}=\Delta-E-k
$$

Performing the integral on $\mu$ leads to

$$
\frac{\mathrm{d} \Gamma_{n \rightarrow}^{\mathrm{BS}}}{\mathrm{d} k \mathrm{~d} E}=\frac{\alpha_{\mathrm{FS}} K}{2 \pi k} E_{\nu}^{2} F_{+}(E, k) .
$$

with

$$
\begin{aligned}
F_{ \pm}(E, k) & \equiv A(E, k) \pm k B(E, k) \\
A(E, k) & \equiv\left(2 E^{2}+k^{2}\right) \ln \left(\frac{E+p}{E-p}\right)-4 p E \\
B(E, k) & \equiv 2 E \ln \left(\frac{E+p}{E-p}\right)-4 p .
\end{aligned}
$$

The total decay rate cannot be obtained from a simple integration of the form

$$
\Gamma_{n \rightarrow}^{\mathrm{BS}}=\int_{m_{e}}^{E_{\max }} \mathrm{d} E \int_{0}^{\Delta-E} \mathrm{~d} k \frac{\mathrm{d} \Gamma^{\mathrm{BS}}}{\mathrm{d} k \mathrm{~d} E} .
$$

since there is an infrared divergence. However this divergence cancels a corresponding divergence in the pure radiative corrections. The usual procedure consists in letting the photon having a mass and taking the limit $m_{\gamma} \rightarrow 0$ [see e.g. Ivanov et al. (2013) or Abers et al. (1968)].

\section{b. Bremsstrahlung for other reactions}

The other rates of bremsstrahlung can be deduced from crossing symmetry. Indeed, if one considers the process $n+e^{+} \rightarrow p+\bar{\nu}$ it is obtained from crossing symmetry of beta decay. A crossing symmetry is performed by inverting the four-momentum of the corresponding particle. Hence changing the position of the final electron to an initial positron amounts to $E \rightarrow-E$ and $p \rightarrow-p$. This leads to $F_{+} \rightarrow F_{-}$in B40. In fact it is simple to check that if the electron (or positron) and the photon are on the same side, one should use $F_{+}$and if they are on opposite sides, we should use $F_{-}$. The contributions of bremsstrahlung to the reaction rates is then straightforward, provided it is clear that we should take the infrared regularized contribution, and they read

$$
\begin{aligned}
\Gamma_{n \rightarrow p \gamma} & =\frac{\alpha_{\mathrm{FS}} K}{2 \pi} \int_{m_{e}}^{\infty} \mathrm{d} E \\
& \times\left[g(-E) \widetilde{\chi}_{+}^{+}(E)+g(E) \widetilde{\chi}_{+}^{-}(-E)\right] \\
\Gamma_{p \rightarrow n \gamma} & =\left.\Gamma_{n \rightarrow p \gamma}\right|_{\tilde{\chi}_{+}^{s} \rightarrow \tilde{\chi}_{-}^{s}}
\end{aligned}
$$

with the definitions

$$
\begin{aligned}
& \tilde{\chi}_{ \pm}^{s}(E) \equiv \int_{0}^{\infty} \frac{\mathrm{d} k}{k} F_{s}(E, k) \tilde{\chi}_{ \pm}(k+E) \\
& \widetilde{\chi}_{ \pm}(E) \equiv\left(E_{\nu}^{\mp}\right)^{2} g_{\nu}^{\mp}\left(E_{\nu}\right), \quad E_{\nu}^{\mp}=E \mp \Delta .
\end{aligned}
$$

\section{c. Correction for bremsstrahlung}

However, the bremsstrahlung radiative corrections included in $\S$ III.E that is in the factor 103, are those of neutron beta decay. Indeed the radiative correction function $R$ in Eqs. (101) and (104) is the sum of a pure radiative correction and a bremsstrahlung correction. It is decomposed as

$$
R\left(E, k_{\max }\right)=R^{\text {pure }}(E)+R^{\mathrm{BS}}\left(E, k_{\max }\right)
$$

with the BS part being

$$
R^{\mathrm{BS}}\left(E, k_{\max }\right) \equiv \frac{\alpha_{\mathrm{FS}}}{2 \pi} \int_{0}^{k_{\max }} \frac{\mathrm{d} k}{k} \frac{\left(k_{\max }-k\right)^{2}}{k_{\max }^{2}} \frac{F_{+}(E, k)}{E p} .
$$

In Eqs. 101 and 104 the choice was made to take $k_{\max }$ as being the energy of the neutrino because this is the case for neutron beta decay. However the maximum energy of the photon emitted is not the neutrino energy in the other reactions. Furthermore, the distribution function for the neutrino appearing in Eqs. (101) and (104) is incorrectly taken in the limit in which the photon energy is so soft that it does not affect the neutrino energy. These are the two shortcomings that we need to correct for by adding the difference between the correct correction [Eqs. (B43)] and the approximate contribution [it is formally Eqs. (101) and 104 with $R \rightarrow R^{\mathrm{BS}}$. We write this bremsstrahlung correction in a form which contains explicitly no infrared divergence and this is given by Eqs. 107) with the definitions 


$$
\begin{aligned}
\delta \Gamma_{n \rightarrow p+e}^{\mathrm{BS}} & =\int_{0}^{|\Delta-E|} \frac{\mathrm{d} k}{k} F_{+}(E, k)\left[\widetilde{\chi}_{+}(E+k)-(|\Delta-E|-k)^{2} g_{\nu}(E-\Delta)\right]+\int_{|\Delta-E|}^{\infty} \frac{\mathrm{d} k}{k} F_{+}(E, k) \widetilde{\chi}_{+}(E+k) \\
\delta \Gamma_{n+e \rightarrow p}^{\mathrm{BS}} & =\int_{0}^{\Delta+E} \frac{\mathrm{d} k}{k}\left[F_{-}(E, k) \widetilde{\chi}_{+}(k-E)-F_{+}(E, k)(|\Delta+E|-k)^{2} g_{\nu}(-E-\Delta)\right] \\
& +\int_{\Delta+E}^{\infty} \frac{\mathrm{d} k}{k} F_{-}(E, k) \widetilde{\chi}_{+}(k-E) \\
\delta \Gamma_{p \rightarrow n+e}^{\mathrm{BS}} & =\int_{0}^{\Delta+E} \frac{\mathrm{d} k}{k} F_{+}(E, k)\left[\widetilde{\chi}_{-}(E+k)-(|E+\Delta|-k)^{2} g_{\nu}(E+\Delta)\right]+\int_{\Delta+E}^{\infty} \frac{\mathrm{d} k}{k} F_{+}(E, k) \widetilde{\chi}_{-}(E+k) \\
\delta \Gamma_{p+e \rightarrow n}^{\mathrm{BS}} & =\int_{0}^{|\Delta-E|} \frac{\mathrm{d} k}{k}\left[F_{-}(E, k) \widetilde{\chi}_{-}(k-E)-F_{+}(E, k)(|E-\Delta|-k)^{2} g_{\nu}(-E+\Delta)\right] \\
& +\int_{|\Delta-E|}^{\infty} \frac{\mathrm{d} k}{k} F_{-}(E, k) \widetilde{\chi}_{-}(k-E) .
\end{aligned}
$$

With these corrections added to Eqs. (101) and 104, the total effect of bremsstrahlung is taken into account. However, with this new contribution the rates no longer satisfy the detailed balance relation (86). Indeed the emission of a final photon needs to be compensated by the absorption of photons from the thermal bath to lead to a thermodynamical equilibrium. It is only when the finite temperature radiative corrections are taken into account, with stimulated emission and absorption, that the total rates can satisfy detailed balance. This is shown explicitly in Fig. 18 .

Note that by construction the bremsstrahlung corrections do not modify the radiative corrections for $\lambda_{0}^{\mathrm{RC} 0}$ because the neutron beta decay in vacuum, is the only reaction for which bremsstrahlung is fully taken into account already.

Finally note that there was some intuition in Brown and Sawyer (2001) about the incorrect treatment of realphoton processes. Indeed in this reference, the authors advocate that one should add the process $n+\bar{\nu}+e^{+} \rightarrow$ $p+\gamma$, that they call the five-body process, since it cannot be a correction to $n+\bar{\nu}+e^{+} \rightarrow p$ which is forbidden energetically. This is indeed correct and it corresponds to the last term in $\delta \Gamma_{n+e \rightarrow p}^{\mathrm{BS}}$, where the photon emitted must have an energy larger than $\Delta+E$. What we find is that not only this process needs to be added, but all other processes need to be corrected except for the neutron beta decay.

\section{Finite temperature radiative corrections}

The finite temperature radiative corrections are made of Eqs. (109), (112) and (113). These last two contributions involve implicitly principal parts in the apparently divergent part of the integrals. After rearrangement, they can be put in the form (Brown and Sawyer, 2001, Eq. $(5.15))$

$$
\begin{aligned}
\Gamma_{n \rightarrow p}^{\Delta E, T}+\Gamma_{n \rightarrow p}^{e p+e e, T}= & \frac{\alpha_{\mathrm{FS}} K}{2 \pi} \int_{m_{e}}^{\infty} \mathrm{d} E\left[\chi_{+}(E)+\chi_{+}(-E)\right]\left[-\frac{2 \pi^{2} T^{2} E}{3 p}+\int_{m_{e}}^{\infty} \mathrm{d} E^{\prime} F^{T}\left(E, E^{\prime}\right)\right] \\
\Gamma_{p \rightarrow n}^{\Delta E, T}+\Gamma_{p \rightarrow n}^{e p+e e, T}= & \left.\left(\Gamma_{n \rightarrow p}^{\Delta E, T}+\Gamma_{n \rightarrow p}^{e p+e e, T}\right)\right|_{\chi+\rightarrow \chi-} \\
F^{T}\left(E, E^{\prime}\right) \equiv & -\frac{1}{4} \ln ^{2}\left(\frac{p+p^{\prime}}{p-p^{\prime}}\right)^{2}\left\{g^{\prime}\left(E^{\prime}\right) \frac{p^{\prime} E^{2}}{p E^{\prime}}\left(E+E^{\prime}\right)+g\left(E^{\prime}\right) \frac{E^{2}}{p p^{\prime}}\left[E^{\prime}+\frac{m_{e}^{2} E}{E^{\prime 2}}\right]\right\}-g\left(E^{\prime}\right)\left[4 E \frac{p^{\prime}}{p}+2 E^{\prime} L\left(E, E^{\prime}\right)\right] \\
& +\ln \left(\frac{p+p^{\prime}}{p-p^{\prime}}\right)^{2}\left\{g^{\prime}\left(E^{\prime}\right)\left[p^{\prime 2} \frac{E}{E^{\prime}}\left(\frac{m_{e}^{2}}{p^{2}}+2\right)-E^{2} \frac{p^{\prime}}{p} L\left[E, E^{\prime}\right]\right]\right. \\
& \left.+g\left(E^{\prime}\right)\left[\frac{E m_{e}^{2}}{p^{2} E^{\prime 2}}\left(E^{\prime 2}+2 p^{2}+m_{e}^{2}\right)-\frac{E^{2}+E^{\prime 2}}{E+E^{\prime}}-\frac{E^{2} E^{\prime}}{p p^{\prime}} L\left(E, E^{\prime}\right)\right]\right\} \\
L\left(E, E^{\prime}\right) \equiv & \ln \left(\frac{E E^{\prime}+p p^{\prime}+m_{e}^{2}}{E E^{\prime}-p p^{\prime}+m_{e}^{2}}\right),
\end{aligned}
$$


where $g^{\prime}(E) \equiv \partial_{E} g(E)$.

This form is simpler numerically if the double integration on $E$ and $E^{\prime}$ is performed on the variables $E_{\Sigma} \equiv E+E^{\prime}$ and $E_{\Delta} \equiv E-E^{\prime}$ using $2 \iint \mathrm{d} E \mathrm{~d} E^{\prime}=$ $\iint \mathrm{d} E_{\Sigma} \mathrm{d} E_{\Delta}$.

The first contribution (109) is made of real photon processes, such as absorption and stimulated emission, see Fig. 14. but not simple emission which has been already accounted for as bremsstrahlung (see appendix B.7). To obtain it we start from the real photons processes (Brown and Sawyer, 2001, Eq. B28)

$$
\begin{aligned}
\Gamma_{n \rightarrow p}^{\gamma} & =\frac{\alpha_{\mathrm{FS}} K}{2 \pi} \int_{m_{e}}^{\infty} \mathrm{d} E \\
& \times\left[g(-E) \widetilde{\chi}_{+}^{\gamma,+}(E)+g(E) \widetilde{\chi}_{+}^{\gamma,-}(-E)\right] \\
\Gamma_{p \rightarrow n}^{\gamma} & =\left.\Gamma_{n \rightarrow p}^{\gamma}\right|_{\widetilde{\chi}_{+}^{\gamma, s} \rightarrow \widetilde{\chi}_{-}^{\gamma, s}}
\end{aligned}
$$

where we defined

$$
\begin{array}{r}
\widetilde{\chi}_{ \pm}^{\gamma, s}(E) \equiv \int_{0}^{\infty} \frac{\mathrm{d} k}{k}\left[(1+f(k)) F_{s}(E, k) \widetilde{\chi}_{ \pm}(E+k)\right. \\
\left.+f(k) F_{-s}(E, k) \widetilde{\chi}_{ \pm}(E-k)\right],(\mathrm{B}
\end{array}
$$

and $f(k) \equiv g^{-}(k / T)=1 /[\exp (k / T)-1]$. Note that from the property

$$
1+f(k)=-f(-k)=\mathrm{e}^{\frac{k}{T}} f(k)
$$

combined with the property (84), the detailed balance relation (86) for the real photon processes $(\bar{B} 52)$ is automatically satisfied ${ }^{21}$.

Then we replace the factor $[1+f(k)]$ by $f(k)$ so as to keep only stimulated emission, since bremsstrahlung processes (B43) which are taken into account separately are obtained by $f(k) \rightarrow 0$. A consequence is that the real photon processes without bremsstrahlung do no satisfy formally the detailed balance relation. It is only when all real photon processes are added that it is recovered, as illustrated in Fig. (18).

Finally, in order to obtain the form 109 for which it is apparent that there is no infrared divergence, it is necessary to add an infrared diverging contribution (Brown and Sawyer, 2001, Eq. (B53)) that can be considered as part of the wave-function radiative correction (it is partially coming from the diagram $17 \mathrm{a}$ and which is

$$
\begin{aligned}
\Gamma_{n \rightarrow p}^{\gamma, Z} & =\frac{\alpha_{\mathrm{FS}} K}{2 \pi} \int_{m_{e}}^{\infty} \mathrm{d} E \\
& \times\left[g(-E) \widetilde{\chi}_{+}^{\gamma, Z}(E)+g(E) \widetilde{\chi}_{+}^{\gamma, Z}(-E)\right] \\
\Gamma_{p \rightarrow n}^{\gamma, Z} & =\left.\Gamma_{n \rightarrow p}^{\gamma, Z}\right|_{\widetilde{\chi}_{+}^{\gamma, Z} \rightarrow \widetilde{\chi}_{-}^{\gamma, Z}}
\end{aligned}
$$

${ }^{21}$ At this stage this is only formal since this presents infrared divergences.

$$
\widetilde{\chi}_{ \pm}^{\gamma, Z}(E) \equiv-2 \int_{0}^{\infty} \frac{\mathrm{d} k}{k} f(k) A(E, k) \widetilde{\chi}_{ \pm}(E) .
$$

Again on Eqs. (B55) it is apparent using the property (84) that these extra terms formally satisfy the detailed balance relation 86 .

\section{Appendix C: Nuclear reactions}

\section{Conventions for nuclear reaction rates}

Let us consider for simplicity a two-body reaction of the type $k+l \rightarrow i+j$ and its reverse reaction $i+j \rightarrow$ $k+l$. From Eqs. (136) and (137) their contributions to the evolution of the abundance $Y_{i}$ take the form

$$
\dot{Y}_{i} \supset Y_{k} Y_{l} \rho_{n} N_{A}\langle\sigma v\rangle_{k l \rightarrow i j}-Y_{i} Y_{j} \rho_{n} N_{A}\langle\sigma v\rangle_{i j \rightarrow k l},
$$

with

$$
\rho_{\mathrm{n}} \equiv \frac{n_{\mathrm{b}}}{N_{A}} \equiv n_{\mathrm{b}} \cdot u\left(\mathrm{~g} / \mathrm{cm}^{3}\right) .
$$

We see that $\rho_{\mathrm{n}}$ has the dimension of a mass density since $u$ is the atomic mass unit, that, by definition is related to Avogadro's number by $N_{A} \cdot u=1 \mathrm{~g}$. By convention, $N_{A}$ has been introduced in the definition of the reaction rate, hence what is tabulated for a given reaction is $N_{A}\langle\sigma v\rangle$ and one must multiply by $\rho_{\mathrm{n}}$ in order to build the rates (e.g. $\Gamma_{k l \rightarrow i j}$ ) which appear in the general form Eq. (138). More generally for a reaction of the type $i_{1}+\cdots+i_{p} \rightarrow$ $j_{1}+\ldots j_{q}$, the contribution to the evolution of the species $i_{1}$ is

$$
\dot{Y}_{i_{1}} \supset-Y_{i_{1}} \ldots Y_{i_{p}} \rho_{\mathrm{n}}^{p-1} N_{A}^{p-1} \gamma_{i_{1} \ldots i_{p} \rightarrow j_{1} \ldots j_{q}} .
$$

Conventionally what is tabulated is $N_{A}^{p-1} \gamma_{i_{1} \ldots i_{p} \rightarrow j_{1} \ldots j_{q}}{ }^{22}$ and one must multiply by $\rho_{\mathrm{n}}^{p-1}$ to build the reaction rate $\Gamma_{i_{1} \ldots i_{p} \rightarrow j_{1} \ldots j_{q}}$ appearing in Eq. 138 . The method is similar for a reaction of the type $j_{1}+\ldots j_{q} \rightarrow i_{1}+\cdots+i_{p}$ which creates species $i_{1}$. See also appendix E of Serpico et al. (2004) on this topic.

To summarize, in nucleosynthesis calculations (as in chemistry) we are concerned with number densities. However, it is convenient to normalize them to Avogadro's number whose dimension can be considered ${ }^{23}$ to be the inverse of a mass. The form (C1) is purely conventional and $\rho_{\mathrm{n}}$ is just another manner to define a number density even though it has the dimension of a mass density.

22 More precisely, from 139 the factor is $N_{A}^{\left(N_{i_{1}}+\cdots+N_{i_{p}}\right)-1}$ if the stoichiometric coefficients are not unity.

${ }^{23}$ A subject of controversy, but irrelevant as soon as it is consistent with the definition of the reaction rate units. 


\section{Baryonic density and nucleonic density}

In practice, except for BBN, the nucleonic density of Eq. C2 is usually identified with the atomic matter density, and the nuclear energy source is calculated independently as nuclear flow $\times \mathrm{Q}$ This corresponds to the approximation

$$
A \cdot u \approx Z m_{p}+(A-Z) m_{n}-B(A, Z)+Z m_{e},
$$

i.e. an error of $\approx 1 \%$, completely negligible in stellar modeling. For $\mathrm{BBN}$ where $\mathrm{D} / \mathrm{H}$ observations reach the percent level of uncertainty, it is worth considering the difference between nucleonic density and baryonic density.

The baryonic density $\Omega_{\mathrm{b}} \cdot h^{2}$ deduced from CMB observations is the atomic density i.e. taking into account the ${ }^{4} \mathrm{He}$ binding energy and the mass of the electrons. Given that the fraction of baryons in the form of helium is $Y_{\mathrm{P}} \equiv 4 Y^{4} \mathrm{He}$ and the rest is in the form of hydrogen, the average mass of baryons is (Steigman, 2006)

$$
\begin{aligned}
m_{\mathrm{b}} & \equiv \xi u \\
\xi & \equiv \frac{Y_{\mathrm{P}}}{4} \frac{m^{4} \mathrm{He}}{u}+\left(1-Y_{\mathrm{P}}\right) \frac{m^{{ }^{1} \mathrm{H}}}{u} \\
& =\frac{m_{{ }^{1} \mathrm{H}}}{u}\left(1-1.75891 \times 10^{-3} \frac{Y_{\mathrm{P}}}{0.24709}\right)
\end{aligned}
$$

where $m_{4} \mathrm{He}$ and $m_{1} \mathrm{H}$ are the atomic masses (see appendix D). Using $Y_{\mathrm{P}} \simeq 0.24709$ for the final BBN Helium abundance (it is the most relevant abundance for $\mathrm{CMB}$ since stellar formation has not started during CMB formation) we get

$$
\xi \simeq 1.006052, \quad \xi^{-1} \simeq 0.993984 .
$$

Hence, from Eqs. (35) and (C2), one should use in Eq. (C1)

$$
\rho_{\mathrm{n}}=\xi^{-1} \rho_{\mathrm{b}},
$$

where $\rho_{\mathrm{b}}$ is obtained from Eq. (34). One can estimate the error introduced if we ignore this subtlety and use $\xi=1$ in nuclear reaction. Numerically we found

$$
\begin{aligned}
\Delta Y_{\mathrm{P}} & =5.6 \times 10^{-4} \\
\Delta\left(\frac{\mathrm{D}}{\mathrm{H}}\right) & =-2.4 \times 10^{-7} \\
\Delta\left(\frac{{ }^{3} \mathrm{He}}{\mathrm{H}}\right) & =3.7 \times 10^{-8} \\
\Delta\left(\frac{{ }^{7} \mathrm{Li}}{\mathrm{H}}\right) & =7.1 \times 10^{-12} .
\end{aligned}
$$

Finally, since the abundances deduced from observations, other than ${ }^{4} \mathrm{He}$, are expressed as number ratios, hence, e.g. the observed $\mathrm{D} / \mathrm{H}$ can be directly compared to the BBN $Y_{\mathrm{D}} / Y^{{ } \mathrm{H}}$ calculated ratio. The ${ }^{4} \mathrm{He}$ (pseudo)mass fraction, $Y_{\mathrm{P}}$ deduced from spectroscopic observations is defined as (Izotov et al., 1994, Pagel et al., 1992)

$$
Y_{\mathrm{P}} \equiv \frac{4 y}{4 y+1}
$$

with $y \equiv n_{4} \mathrm{He} / n_{{ }_{1} \mathrm{H}} \equiv Y^{4} \mathrm{He} / Y_{{ }^{1} \mathrm{H}}$ being the number ratio, resulting in a definition of $Y_{\mathrm{P}}$ identical to the BBN one.

\section{Appendix D: Numerical values}

The values of $\cos \theta_{C}=V_{\mathrm{ud}}$ and $g_{A}=C_{A} / C_{V}$ are taken from the Particle Data Group (PDG) (Patrignani and Particle Data Group 2016 and 2017 update). The neutron decay rate $\tau_{n}=879.5( \pm 0.8) \mathrm{s}$ is from Serebrov et al. (2017, Fig. 22) and includes only experiments after 2000. This value is slightly lower and with less experimental error than the previously admitted value $\tau_{n}=880.2(1.1) \mathrm{s}$ of the PDG. We report errors on numerical parameters only if they are meaningful for BBN. Cosmological parameters are taken from Ade et al. (2016).

TABLE X Numerical values used for the BBN code.

\begin{tabular}{ll}
\hline$u$ & $931.494061 \mathrm{MeV}$ \\
$m_{n}$ & $939.565360 \mathrm{MeV}$ \\
$m_{p}$ & $938.272029 \mathrm{MeV}$ \\
$m_{Z}$ & $91.1876 \mathrm{GeV}$ \\
$m_{W}$ & $80.385 \mathrm{GeV}$ \\
$m_{4} \mathrm{He}$ (atomic) & $4.00260325413 u$ \\
$m_{1_{\mathrm{H}}}($ atomic $)$ & $1.00782503223 u$ \\
\hline$g_{A}$ & $1.2723(23)$ \\
$\cos \theta_{C}$ & $0.97420(20)$ \\
$f_{\mathrm{WM}}$ & 1.853 \\
$G_{F}$ & $1.1663787 \times 10^{-5} \mathrm{GeV}^{-2}$ \\
$\tau_{n}$ & $879.5(8) \mathrm{s}$ \\
$r_{p}$ & $0.841 \times 10^{-15} \mathrm{~m}$ \\
$\alpha_{\mathrm{FS}}$ & $1 / 137.03599911$ \\
\hline$T_{0}$ & $2.7255( \pm 0.0006) \mathrm{K}$ \\
$h=H / H_{100}$ & $0.6727( \pm 0.0066)$ \\
$h^{2} \Omega_{b}$ & $0.022250( \pm 0.00016)$ \\
$h^{2} \Omega_{c}$ & $0.1198( \pm 0.0015)$ \\
$\rho_{100}^{\text {crit }} \equiv 3 H_{100}^{2} /(8 \pi G)$ & $1.87847 \times 10^{-29} \mathrm{~g} / \mathrm{cm}^{3}$ \\
\hline &
\end{tabular}

\section{References}

Abers, E. S., D. A. Dicus, R. E. Norton, and H. R. Quinn, 1968, Phys. Rev. 167, 1461.

Ade, P. A. R., et al. (Planck), 2016, Astron. Astrophys. 594, A13.

Adelberger, E. G., A. García, R. G. H. Robertson, K. A. Snover, A. B. Balantekin, K. Heeger, M. J. Ramsey-Musolf, D. Bemmerer, A. Junghans, C. A. Bertulani, J.-W. Chen, H. Costantini, et al., 2011, Rev. Mod. Phys. 83, 195.

Aliotta, M., F. Raiola, G. Gyürky, A. Formicola, R. Bonetti, C. Broggini, L. Campajola, P. Corvisiero, H. Costantini, A. D'Onofrio, Z. Fülöp, G. Gervino, et al., 2001, Nuclear Physics A 690, 790.

Amsler, C., M. Doser, M. Antonelli, D. M. Asner, K. S. Babu, H. Baer, H. R. Band, R. M. Barnett, E. Bergren, 
J. Beringer, G. Bernardi, W. Bertl, et al., 2008, Physics Letters B 667, 1.

Anders, M., D. Trezzi, R. Menegazzo, M. Aliotta, A. Bellini, D. Bemmerer, C. Broggini, A. Caciolli, P. Corvisiero, H. Costantini, T. Davinson, Z. Elekes, et al., 2014, Phys, Rev. Lett. 113, 042501.

Ando, S., R. H. Cyburt, S. W. Hong, and C. H. Hyun, 2006, Phys. Rev. C74, 025809.

Angulo, C., M. Arnould, M. Rayet, P. Descouvemont, D. Baye, C. Leclercq-Willain, A. Coc, S. Barhoumi, P. Aguer, C. Rolfs, R. Kunz, J. W. Hammer, et al., 1999, Nuclear Physics A 656, 3.

Angulo, C., E. Casarejos, M. Couder, P. Demaret, P. Leleux, F. Vanderbist, A. Coc, J. Kiener, V. Tatischeff, T. Davinson, A. S. Murphy, N. L. Achouri, et al., 2005, Astrophys. J. Lett. 630, L105.

Aoki, W., P. S. Barklem, T. C. Beers, N. Christlieb, S. Inoue, A. E. García Pérez, J. E. Norris, and D. Carollo, 2009, Astrophys. J. 698, 1803.

Arai, K., S. Aoyama, Y. Suzuki, P. Descouvemont, and D. Baye, 2011, Phys. Rev. Lett. 107, 132502.

Arbey, A., 2012, Comput. Phys. Commun. 183, 1822.

Asplund, M., D. L. Lambert, P. E. Nissen, F. Primas, and V. V. Smith, 2006, Astrophys. J. 644, 229.

Audi, G., F. G. Kondev, M. Wang, W. J. Huang, and S. Naimi, 2017, Chinese Physics C 41, 030001.

Aver, E., K. A. Olive, and E. D. Skillman, 2015, "JCAP" 7, 011.

Balashev, S. A., E. O. Zavarygin, A. V. Ivanchik, K. N. Telikova, and D. A. Varshalovich, 2016, MNRAS 458, 2188.

Bania, T. M., R. T. Rood, and D. S. Balser, 2002, Nature 415, 54.

Barbagallo, M., A. Musumarra, L. Cosentino, E. Maugeri, S. Heinitz, A. Mengoni, R. Dressler, D. Schumann, F. Käppeler, N. Colonna, P. Finocchiaro, M. Ayranov, et al., 2016, Phys. Rev. Lett. 117, 152701.

Becchetti, F. D., J. A. Brown, W. Z. Liu, J. W. Jänecke, D. A. Roberts, J. J. Kolata, R. J. Smith, K. Lamkin, A. Morsad, R. E. Warner, R. N. Boyd, and J. D. Kalen, 1992, Nucl. Phys. A 550, 507.

Bernstein, J., L. S. Brown, and G. Feinberg, 1989, Rev. Mod. Phys. 61, 25.

Birrell, J., C.-T. Yang, and J. Rafelski, 2014, Nucl. Phys. B890, 481.

Blas, D., J. Lesgourgues, and T. Tram, 2011, JCAP 7, 034.

Bonifacio, P., P. Molaro, T. Sivarani, R. Cayrel, M. Spite, F. Spite, B. Plez, J. Andersen, B. Barbuy, T. C. Beers, E. Depagne, V. Hill, et al., 2007, Astron. Astrophys. 462, 851.

Broggini, C., L. Canton, G. Fiorentini, and F. L. Villante, 2012, JCAP 6, 030.

Brown, L. S., and R. F. Sawyer, 2001, Phys. Rev. D63, 083503.

Bystritsky, V. M., V. V. Gerasimov, A. R. Krylov, S. S. Parzhitskii, G. N. Dudkin, V. L. Kaminskii, B. A. Nechaev, V. N. Padalko, A. V. Petrov, G. A. Mesyats, M. Filipowicz, J. Wozniak, et al., 2008, Nuclear Instruments and Methods in Physics Research A 595, 543.

Cambier, J.-L., J. R. Primack, and M. Sher, 1982, Nucl. Phys. B209, 372 .

Casella, C., H. Costantini, A. Lemut, B. Limata, R. Bonetti, C. Broggini, L. Campajola, P. Corvisiero, J. Cruz, A. D'Onofrio, A. Formicola, Z. Fülöp, et al., 2002, Nucl. Phys. A 706, 203.
Cassisi, S., and V. Castellani, 1993, Astrophys. J. Supp. 88, 509.

Chakraborty, N., B. D. Fields, and K. A. Olive, 2011, Phys. Rev. D83, 063006.

Chapman, I. A., 1997, Phys. Rev. D55, 6287.

Charbonnel, C., and F. Primas, 2005, Astron. Astrophys. 442, 961.

Clayton, D. D., 1983, Principles of stellar evolution and nucleosynthesis (Chicago: University of Chicago Press).

Coc, A., 2013, Acta Physica Polonica B 44, 521.

Coc, A., S. Goriely, Y. Xu, M. Saimpert, and E. Vangioni, 2012, Astrophys. J. 744, 158.

Coc, A., F. Hammache, and J. Kiener, 2015, European Physical Journal A 51, 34.

Coc, A., K. A. Olive, J.-P. Uzan, and E. Vangioni, 2009, Phys. Rev. D79, 103512.

Coc, A., P. Petitjean, J.-P. Uzan, E. Vangioni, P. Descouvemont, C. Iliadis, and R. Longland, 2015, Phys. Rev. D92, 123526 .

Coc, A., J.-P. Uzan, and E. Vangioni, 2014, JCAP 1410, 050.

Coc, A., and E. Vangioni, 2010, in Big-Bang nucleosynthesis with updated nuclear data, volume 202 of Journal of Physics Conference Series, p. 012001.

Coc, A., and E. Vangioni, 2014, in Proceedings, XIII Nuclei in the Cosmos, Debrecen, Hungary, July 7-11, 2014, PoS (NIC XIII), p. 22.

Coc, A., and E. Vangioni, 2017, International Journal of Modern Physics E 26, 1741002.

Coc, A., E. Vangioni-Flam, M. Cassé, and M. Rabiet, 2002, Phys. Rev. D65, 043510.

Coc, A., E. Vangioni-Flam, P. Descouvemont, A. Adahchour, and C. Angulo, 2004, Astrophys. J. 600, 544.

Consiglio, R., P. F. de Salas, G. Mangano, G. Miele, S. Pastor, and O. Pisanti, 2017, eprint 1712.04378.

Cooke, R. J., 2015, Astrophys. J. Lett. 812, L12.

Cooke, R. J., M. Pettini, R. A. Jorgenson, M. T. Murphy, and C. C. Steidel, 2014, Astrophys. J. 781, 31.

Cooke, R. J., M. Pettini, K. M. Nollett, and R. Jorgenson, 2016, Astrophys. J. 830, 148.

Cooke, R. J., M. Pettini, and C. C. Steidel, 2018, Astrophys. J. 855, 102.

Cooper, R. L., T. E. Chupp, M. S. Dewey, T. R. Gentile, H. P. Mumm, J. S. Nico, A. K. Thompson, B. M. Fisher, I. Kremsky, F. E. Wietfeldt, E. J. Beise, K. G. Kiriluk, et al., 2010, Phys. Rev. C81, 035503.

Creminelli, P., C. Pitrou, and F. Vernizzi, 2011, JCAP 1111, 025.

Cyburt, R. H., 2004, Phys. Rev. D70, 023505.

Cyburt, R. H., and B. Davids, 2008, Phys. Rev. C78, 064614.

Cyburt, R. H., B. D. Fields, and K. A. Olive, 2008, JCAP 11, 012.

Cyburt, R. H., B. D. Fields, K. A. Olive, and T.-H. Yeh, 2016, Rev. Mod. Phys. 88, 015004.

Cyburt, R. H., and M. Pospelov, 2012, International Journal of Modern Physics E 21, 1250004-1-1250004-13.

Czarnecki, A., W. J. Marciano, and A. Sirlin, 2004, Phys. Rev. D70, 093006.

Czarnecki, A., W. J. Marciano, and A. Sirlin, 2018, Phys. Rev. Lett. 120, 202002.

deBoer, R. J., J. Görres, K. Smith, E. Uberseder, M. Wiescher, A. Kontos, G. Imbriani, A. Di Leva, and F. Strieder, 2014, Phys. Rev. C90, 035804.

Descouvemont, P., A. Adahchour, C. Angulo, A. Coc, and E. Vangioni-Flam, 2004, Atomic Data and Nuclear Data 
Tables 88, 203.

Di Valentino, E., C. Gustavino, J. Lesgourgues, G. Mangano, A. Melchiorri, G. Miele, and O. Pisanti, 2014, Phys. Rev. D90(2), 023543.

Dicus, D. A., E. W. Kolb, A. M. Gleeson, E. C. G. Sudarshan, V. L. Teplitz, and M. S. Turner, 1982, Phys. Rev. D26, 2694.

Dodelson, S., and M. S. Turner, 1992, PHys. Rev. D46, 3372.

Dolgov, A. D., S. H. Hansen, S. Pastor, S. T. Petcov, G. G. Raffelt, and D. V. Semikoz, 2002, Nucl. Phys. B632, 363.

Dolgov, A. D., S. H. Hansen, and D. V. Semikoz, 1997, Nucl. Phys. B503, 426.

Dolgov, A. D., S. H. Hansen, and D. V. Semikoz, 1999, Nucl. Phys. B543, 269.

Dvorkin, I., E. Vangioni, J. Silk, P. Petitjean, and K. A. Olive, 2016, MNRAS 458, L104.

Ekström, S., G. Meynet, C. Chiappini, R. Hirschi, and A. Maeder, 2008, Astron. Astrophys. 489, 685.

Esposito, S., G. Mangano, G. Miele, and O. Pisanti, 1999, Nucl. Phys. B540, 3.

Esposito, S., G. Mangano, G. Miele, and O. Pisanti, 2000a, Nucl. Phys. B568, 421.

Esposito, S., G. Miele, S. Pastor, M. Peloso, and O. Pisanti, 2000b, Nucl. Phys. B590, 539.

Famiano, M. A., A. B. Balantekin, and T. Kajino, 2016, Phys. Rev. C93, 045804.

Fidler, C., and C. Pitrou, 2017, JCAP 1706(06), 013.

Fields, B. D., 2011, Annual Review of Nuclear and Particle Science 61, 47 .

Fields, B. D., S. Dodelson, and M. S. Turner, 1993, Phys. Rev. D47, 4309.

Fornal, B., and B. Grinstein, 2018, ArXiv e-prints eprint 1801.01124.

Fornengo, N., C. W. Kim, and J. Song, 1997, Phys. Rev. D56, 5123.

Fowler, W. A., G. R. Caughlan, and B. A. Zimmerman, 1967, Ann. Rev. of Astron. and Astrophys. 5, 525.

Fröberg, C.-E., 1955, Reviews of Modern Physics 27, 399.

Fu, X., A. Bressan, P. Molaro, and P. Marigo, 2015, MNRAS 452, 3256.

Fu, X., D. Romano, A. Bragaglia, A. Mucciarelli, K. Lind, E. Delgado Mena, S. G. Sousa, S. Randich, A. Bressan, L. Sbordone, S. Martell, A. J. Korn, et al., 2018, Astron. Astrophys. 610, A38.

Gnedin, N. Y., and O. Y. Gnedin, 1998, Astrophys. J. 509, 11.

Gómez Iñesta, Á., C. Iliadis, and A. Coc, 2017, ArXiv e-prints eprint 1710.01647 .

Goriely, S., S. Hilaire, and A. J. Koning, 2008, Astron. Astrophys. 487, 767.

Goudelis, A., M. Pospelov, and J. Pradler, 2016, Phys. Rev. Lett. 116, 211303.

Greife, U., F. Gorris, M. Junker, C. Rolfs, and D. Zahnow, 1995, Zeitschrift fur Physik A Hadrons and Nuclei 351, 107.

Grohs, E., G. M. Fuller, C. T. Kishimoto, and M. W. Paris, 2017, Phys. Rev. D95, 063503.

Grohs, E., G. M. Fuller, C. T. Kishimoto, M. W. Paris, and A. Vlasenko, 2016, Phys. Rev. D93, 083522.

Gruyters, P., K. Lind, O. Richard, F. Grundahl, M. Asplund, L. Casagrande, C. Charbonnel, A. Milone, F. Primas, and A. J. Korn, 2016, Astron. Astrophys. 589, A61.

Gustavino, C., 2017, in European Physical Journal Web of Conferences, volume 136, p. 01009.
Hammache, F., A. Coc, N. de Séréville, I. Stefan, P. Roussel, S. Ancelin, M. Assié, L. Audouin, D. Beaumel, S. Franchoo, B. Fernandez-Dominguez, S. Fox, et al., 2013, Phys. Rev. C88, 062802.

Hammache, F., M. Heil, S. Typel, D. Galaviz, K. Sümmerer, A. Coc, F. Uhlig, F. Attallah, M. Caamano, D. Cortina, H. Geissel, M. Hellström, et al., 2010, Phys. Rev. C82, 065803.

Hannestad, S., 2002, Phys. Rev. D65, 083006.

Hannestad, S., and J. Madsen, 1995, Phys. Rev. D52, 1764.

Heckler, A. F., 1994, Phys. Rev. D49, 611.

Hernanz, M., J. Jose, A. Coc, and J. Isern, 1996, Astrophys. J. Lett. 465, L27.

Horowitz, C. J., 2002, Phys. Rev. D65, 043001.

Horowitz, C. J., and G. Li, 2000, Phys. Rev. D61, 063002.

Hosford, A., S. G. Ryan, A. E. García Pérez, J. E. Norris, and K. A. Olive, 2009, Astron. Astrophys. 493, 601.

Hou, S. Q., J. J. He, S. Kubono, and Y. S. Chen, 2015, Phys. Rev. C91, 055802.

Howk, J. C., N. Lehner, B. D. Fields, and G. J. Mathews, 2012, Nature 489, 121.

Iliadis, C., 2007, Nuclear Physics of Stars (Wiley-VCH Verlag).

Iliadis, C., K. S. Anderson, A. Coc, F. X. Timmes, and S. Starrfield, 2016, Astrophys. J. 831, 107.

Iliadis, C., R. Longland, A. E. Champagne, A. Coc, and R. Fitzgerald, 2010, Nucl. Phys. A841, 31.

Iocco, F., G. Mangano, G. Miele, O. Pisanti, and P. D. Serpico, 2007, Phys. Rev. D75, 087304.

Iocco, F., G. Mangano, G. Miele, O. Pisanti, and P. D. Serpico, 2009, Phys. Rep. 472, 1.

Ivanov, A. N., R. Höllwieser, N. I. Troitskaya, M. Wellenzohn, and Ya. A. Berdnikov, 2017, Phys. Rev. D95, 033007.

Ivanov, A. N., M. Pitschmann, and N. I. Troitskaya, 2013, Phys. Rev. D88, 073002 .

Izotov, Y. I., T. X. Thuan, and N. G. Guseva, 2014, MNRAS 445, 778

Izotov, Y. I., T. X. Thuan, and V. A. Lipovetsky, 1994, Astrophys. J. 435, 647.

Izzo, L., M. Della Valle, E. Mason, F. Matteucci, D. Romano, L. Pasquini, L. Vanzi, A. Jordan, J. M. Fernandez, P. Bluhm, R. Brahm, N. Espinoza, et al., 2015, Astrophys. J. Lett. 808, L14.

Kawabata, T., Y. Fujikawa, T. Furuno, T. Goto, T. Hashimoto, M. Ichikawa, M. Itoh, N. Iwasa, Y. KanadaEn'yo, A. Koshikawa, S. Kubono, E. Miyawaki, et al., 2017, Phys. Rev. Lett. 118, 052701.

Kawano, L., 1992, Let's go: Early universe. 2. Primordial nucleosynthesis: The Computer way.

Kernan, P. J., 1993, Two astroparticle physics problems: Solar neutrinos and primordial He-4, Ph.D. thesis, Ohio State University.

Kirsebom, O. S., and B. Davids, 2011, Phys. Rev. C84, 058801.

Korn, A. J., F. Grundahl, O. Richard, P. S. Barklem, L. Mashonkina, R. Collet, N. Piskunov, and B. Gustafsson, 2006, Nature 442, 657.

Kusakabe, M., M.-K. Cheoun, and K. S. Kim, 2014, Phys. Rev. D90, 045009.

Lesgourgues, J., 2011, CLASS, http://class-code.net/

Lewis, A., and A. Challinor, 1999, CAMB, http://camb. info

Lewis, A., A. Challinor, and A. Lasenby, 2000, Astrophys. J. $\mathbf{5 3 8}, 473$. 
Lind, K., J. Melendez, M. Asplund, R. Collet, and Z. Magic, 2013, Astron. Astrophys. 554, A96.

Longland, R., C. Iliadis, A. E. Champagne, J. R. Newton, C. Ugalde, A. Coc, and R. Fitzgerald, 2010, Nucl. Phys. A841, 1.

Lopez, R. E., and M. S. Turner, 1999, Phys. Rev. D59, 103502.

Lopez, R. E., M. S. Turner, and G. Gyuk, 1997, Phys. Rev. D56, 3191.

Ma, L., H. J. Karwowski, C. R. Brune, Z. Ayer, T. C. Black, J. C. Blackmon, E. J. Ludwig, M. Viviani, A. Kievsky, and R. Schiavilla, 1997, Phys. Rev. C55, 588.

Mangano, G., G. Miele, S. Pastor, and M. Peloso, 2002, Phys. Lett. B534, 8.

Mangano, G., G. Miele, S. Pastor, T. Pinto, O. Pisanti, and P. D. Serpico, 2005, Nucl. Phys. B729, 221.

Mangano, G., G. Miele, S. Pastor, T. Pinto, O. Pisanti, and P. D. Serpico, 2006, Nucl. Phys. B756, 100.

Marciano, W. J., and A. Sirlin, 2006, Phys. Rev. Lett. 96, 032002.

Marcucci, L. E., G. Mangano, A. Kievsky, and M. Viviani, 2016, Phys. Rev. Lett. 116, 102501.

Marcucci, L. E., M. Viviani, R. Schiavilla, A. Kievsky, and S. Rosati, 2005, Phys. Rev. C72, 014001.

Mathews, G. J., and M. Kusakabe, 2017, International Journal of Modern Physics E 26, 1702006.

Mathews, G. J., M. Kusakabe, and T. Kajino, 2017, International Journal of Modern Physics E 26, 1741001.

Meléndez, J., L. Casagrande, I. Ramírez, M. Asplund, and W. J. Schuster, 2010, Astron. Astrophys. 515, L3.

Mendes, D. R., Jr., A. Lépine-Szily, P. Descouvemont, R. Lichtenthäler, V. Guimarães, P. N. de Faria, A. Barioni, K. C. C. Pires, V. Morcelle, R. Pampa Condori, M. C. Morais, E. Leistenschneider, et al., 2012, Phys. Rev. C86, 064321.

Michaud, G., G. Fontaine, and G. Beaudet, 1984, Astrophys. J. 282, 206.

Mirbabayi, M., and M. Zaldarriaga, 2015, JCAP 1503(03), 056 .

Mukhamedzhanov, A. M., Shubhchintak, and C. A. Bertulani, 2016, Phys. Rev. C93, 045805.

Nakamura, R., M.-a. Hasahimoto, R. Ichimasa, and K. Arai, 2017, Int. J. Mod. Phys. E26(08), 1741003.

Neff, T., 2011, Phys. Rev. Lett. 106, 042502.

Nollett, K. M., and S. Burles, 2000, PHys. Rev. D61, 123505.

Oldengott, I. M., and D. J. Schwarz, 2017, Europhys. Lett. $\mathbf{1 1 9}(2), 29001$

Olive, K. A., 2010, ArXiv e-prints eprint 1005.3955.

Olive, K. A., P. Petitjean, E. Vangioni, and J. Silk, 2012, MNRAS 426, 1427.

O'Malley, P. D., D. W. Bardayan, A. S. Adekola, S. Ahn, K. Y. Chae, J. A. Cizewski, S. Graves, M. E. Howard, K. L. Jones, R. L. Kozub, L. Lindhardt, M. Matos, et al., 2011, Phys. Rev. C84, 042801.

Pagel, B. E. J., E. A. Simonson, R. J. Terlevich, and M. G. Edmunds, 1992, Mon. Not. R. Astron. Soc. 255, 325.

Patrignani, C., and Particle Data Group, 2016 and 2017 update, Chinese Physics C 40, 100001.

Pettini, M., and R. Cooke, 2012, MNRAS 425, 2477.

Pisanti, O., A. Cirillo, S. Esposito, F. Iocco, G. Mangano, G. Miele, and P. D. Serpico, 2008, Comput. Phys. Commun. 178, 956 .

Pitrou, C., and A. Stebbins, 2014, Gen. Rel. Grav. 46(11), 1806.
Pospelov, M., and J. Pradler, 2010, Annual Review of Nuclear and Particle Science 60, 539.

Reggiani, H., J. Meléndez, C. Kobayashi, A. Karakas, and V. Placco, 2017, Astron. Astrophys. 608, A46.

Richard, O., G. Michaud, and J. Richer, 2005, Astrophys. J. 619, 538 .

Riemer-Sørensen, S., S. Kotuš, J. K. Webb, K. Ali, V. Dumont, M. T. Murphy, and R. F. Carswell, 2017, MNRAS 468, 3239.

de Salas, P. F., and S. Pastor, 2016, JCAP 1607(07), 051.

Sallaska, A. L., C. Iliadis, A. E. Champange, S. Goriely, S. Starrfield, and F. X. Timmes, 2013, Astrophys. J. 207, 18.

Sawyer, R. F., 1996, Phys. Rev. D53, 4232.

Sbordone, L., P. Bonifacio, E. Caffau, H.-G. Ludwig, N. T. Behara, J. I. González Hernández, M. Steffen, R. Cayrel, B. Freytag, C. van't Veer, P. Molaro, B. Plez, et al., 2010, Astron. Astrophys. 522, A26.

Schaeuble, M., and J. R. King, 2012, PASP 124, 164.

Schmid, G. J., B. J. Rice, R. M. Chasteler, M. A. Godwin, G. C. Kiang, L. L. Kiang, C. M. Laymon, R. M. Prior, D. R. Tilley, and H. R. Weller, 1997, Phys. Rev. C56, 2565.

Scholl, C., Y. Fujita, T. Adachi, P. von Brentano, H. Fujita, M. Górska, H. Hashimoto, K. Hatanaka, H. Matsubara, K. Nakanishi, T. Ohta, Y. Sakemi, et al., 2011, Phys. Rev. C84, 014308.

Scholz, R.-D., U. Heber, C. Heuser, E. Ziegerer, S. Geier, and F. Niederhofer, 2015, Astron. Astrophys. 574, A96.

Seckel, D., 1993, eprint hep-ph/9305311.

Serebrov, A. P., et al., 2017, eprint 1712.05663.

Serpico, P. D., S. Esposito, F. Iocco, G. Mangano, G. Miele, and O. Pisanti, 2004, JCAP 0412, 010.

Serpico, P. D., and G. G. Raffelt, 2005, Phys. Rev. D71, 127301.

Simha, V., and G. Steigman, 2008, JCAP 0808, 011.

Simonucci, S., S. Taioli, S. Palmerini, and M. Busso, 2013, Astrophys. J. 764, 118.

Sirlin, A., 1967, Phys. Rev. 164, 1767.

Smith, C. J., and G. M. Fuller, 2010, Phys. Rev. D81, 065027.

Spite, F., and M. Spite, 1982, Astron. Astrophys. 115, 357.

Spite, M., F. Spite, and P. Bonifacio, 2012, Memorie della Societa Astronomica Italiana Supplementi 22, 9.

Spite, M., F. Spite, E. Caffau, and P. Bonifacio, 2015, Astron. Astrophys. 582, A74.

Steigman, G., 2006, JCAP 0610, 016.

Steigman, G., 2007, Annual Review of Nuclear and Particle Science 57, 463.

Tajitsu, A., K. Sadakane, H. Naito, A. Arai, H. Kawakita, and W. Aoki, 2016, Astrophys. J. 818, 191.

Vangioni-Flam, E., A. Coc, and M. Cassé, 2000, Astron. Astrophys. 360, 15.

Vangioni-Flam, E., K. A. Olive, B. D. Fields, and M. Cassé, 2003, Astrophys. J. 585, 611.

Vernizzi, F., 2005, Phys. Rev. D71, 061301.

Wagoner, R. V., 1969, Astrophys. J. Supp. 18, 247.

Wagoner, R. V., 1973, Astrophys. J. 179, 343.

Wagoner, R. V., W. A. Fowler, and F. Hoyle, 1967, Astrophys. J. Supp. 148, 3.

Wang, B., C. A. Bertulani, and A. B. Balantekin, 2011, Phys. Rev. C83, 018801.

Weinberg, S., 1972, Gravitation and Cosmology: Principles and Applications of the General Theory of Relativity (Wiley).

Weinberg, S., 2003, Phys. Rev. D67, 123504. 
Wietfeldt, F. E., and G. L. Greene, 2011, Rev. Mod. Phys. 83, 1173.

Wilkinson, D. H., 1982, Nucl. Phys. A377, 474.

Wong, Y. Y. Y., 2002, Phys. Rev. D66, 025015.

$\mathrm{Xu}$, Y., K. Takahashi, S. Goriely, M. Arnould, M. Ohta, and H. Utsunomiya, 2013, Nucl. Phys. A918, 61.

Yamazaki, D. G., M. Kusakabe, T. Kajino, G. J. Mathews, and M.-K. Cheoun, 2014, Phys. Rev. D90, 023001.

Young, A. R., S. Clayton, B. W. Filippone, P. Geltenbort, T. M. Ito, C.-Y. Liu, M. Makela, C. L. Morris, B. Plaster, A. Saunders, S. J. Seestrom, and R. B. Vogelaar, 2014, Journal of Physics G Nuclear Physics 41, 114007.

Zavarygin, E. O., J. K. Webb, V. Dumont, and S. RiemerSørensen, 2017, ArXiv e-prints eprint 1706.09512.

Zavarygin, E. O., J. K. Webb, S. Riemer-Sørensen, and V. Dumont, 2018, ArXiv e-prints eprint 1801.04704. 